Mechanism Design for the Allocation of Networked Resources

A Dissertation
Presented to
the faculty of the School of Engineering and Applied Science
University of Virginia
in partial fulfillment
of the requirements for the degree
Doctor of Philosophy
by
Jorge I. Barrera Alviar

May

2013 
APPROVAL SHEET

The dissertation

is submitted in partial fulfillment of the requirements

for the degree of

Doctor of Philosophy

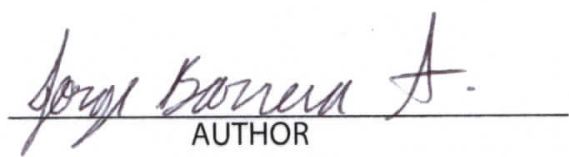

The dissertation has been read and approved by the examining committee:

\begin{tabular}{c} 
Alfredo Garcia \\
\hline $\begin{array}{c}\text { Advisor } \\
\text { Randy Cogill }\end{array}$ \\
\hline Ennio Stacchetti \\
\hline Peter Beling \\
\hline Stephen Wilson
\end{tabular}

Accepted for the School of Engineering and Applied Science:

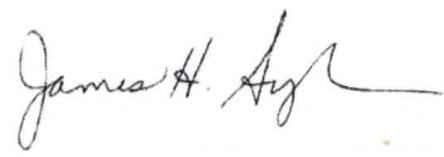

Dean, School of Engineering and Applied Science

May

2013 


\title{
Mechanism Design for the Allocation of Networked Resources
}

\author{
A Dissertation \\ Presented to \\ the Faculty of the School of Engineering and Applied Science \\ University of Virginia \\ In Partial Fulfillment \\ of the requirements for the Degree \\ Doctor of Philosophy (Systems and Information Engineering) \\ by \\ Jorge Barrera Alviar
}

May 2013 


\section{Approval Sheet}

This dissertation is submitted in partial fulfillment of the requirements for the degree of

Doctor of Philosophy (Systems and Information Engineering)

Jorge Barrera Alviar

This dissertation has been read and approved by the Examining Committee:

$$
\text { Alfredo Garcia, Advisor }
$$

Randy Cogill, Committee Chair

Ennio Stacchetti

Peter A. Beling

Stephen G. Wilson

Accepted for the School of Engineering and Applied Science:

Dean, School of Engineering and Applied Science

May 2013 


\section{Acknowledgment}

I want to express special gratitude towards my advisor, Dr. Alfredo Garcia, whose enthusiasm and strong commitment with excellence has shaped not only this work but myself as a professional and as a person. I also want to thank professors Dr. Randy Cogill, Dr. Ennio Stacchetti, Dr. Peter Beling and Dr. Stephen Wilson, who generously accepted to be in my committee and whose guidance has been most helpful.

Not only has this been an academic journey, but a personal one. I feel deeply grateful to David, Juan Manuel, Ana Isabel, Italo, Irwin, Mingyi, Andriy, Mark, Kelly, Dr. Patek, and others who in different ways have enhanced my experience as a graduate student.

This acknowledgment would be incomplete without mentioning the support of my family. Those who, often silently, showed me the peace of rightfulness and the power of persistence.

Finally, I want to thank God for the opportunities I have been given. 


\section{Contents}

1 Introduction $\quad 3$

1.0.1 Mechanism Design in Communication Networks . . . . . . 5

1.0.2 A Brief Introduction to the Theory of Mechanism Design . . 6

1.0.3 Contributions of this Thesis . . . . . . . . . . . 11

2 Inertial Capacity-Auction $\quad \mathbf{1 5}$

2.1 Preliminaries . . . . . . . . . . . . . . . . . 15

2.1 .1 Introduction . . . . . . . . . . . . . . . . . 15

2.1.2 Basic Framework . . . . . . . . . . . . . . . 15

2.2 Literature Review . . . . . . . . . . . . . . . . . . . . . . . 18

2.3 Inertial Capacity Auction (ICA) . . . . . . . . . . . . . . 23

2.3.1 About the Increasing Demand Inertia $\alpha_{t} \ldots \ldots \ldots$

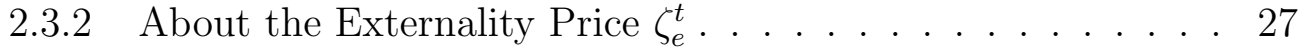

2.3.3 About Payment Rule . . . . . . . . . . . . . . . . . 27

2.4 Assumptions . . . . . . . . . . . . . . . . . . . . . 28

2.5 Price and Flow profile Convergence . . . . . . . . . . . . . . . 29

2.5.1 Convergence of Control Price Profile $\boldsymbol{\mu}^{t} \ldots \ldots$. . . . . . . . 29

2.5.2 Convergence of Flow profile $\mathbf{r}^{t}$ and Efficiency of the Limit

Configuration .................... 31

2.6 Incentive Compatibility . . . . . . . . . . . . . . . . . . 34

2.7 Conclusion . . . . . . . . . . . . . . . . . . . . . . . . . . . 39

2.8 Appendix of Inertial Capacity Auction . . . . . . . . . . . . . . . . 40

2.8.1 Proof of Lemma 2.1 . . . . . . . . . . . . . . . . . . . 40

2.8.2 Proof of Corollary $2.2 \ldots \ldots \ldots$. . . . . . . . . . . . 41

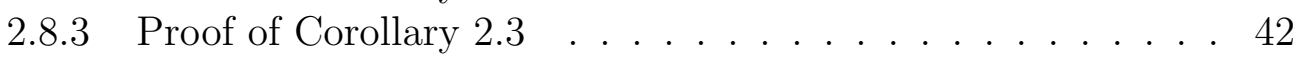

2.8.4 Proof of Lemma 2.2 . . . . . . . . . . . . . . . 43

3 Uplink-Temperature Inertial Auction 45

3.1 Preliminaries ......................... 45

3.1 .1 Introduction . . . . . . . . . . . . . . . . 45

3.1 .2 Basic Framework . . . . . . . . . . . . . . 46

3.2 Literature Review . . . . . . . . . . . . . . . . . . . . . . 50

3.3 Inertial Temperature Auction . . . . . . . . . . . . . . . 54

3.3.1 Notes about the auction design . . . . . . . . . . . . 56

3.3.2 About externality price . . . . . . . . . . . . 57

3.3 .3 About inertia . . . . . . . . . . . . . . 58

3.3.4 About payment rule .................. 58 
3.4 Assumptions . . . . . . . . . . . . . . . . . . . 60

3.5 Convergence of Prices and Joint Power Profile . . . . . . . . . . . . 61

3.5.1 Power profile convergence and efficiency . . . . . . . . . . 62

3.6 Incentive Compatibility . . . . . . . . . . . . . . . . . . 65

3.7 Conclusion . . . . . . . . . . . . . . . . . . . 70

3.8 Appendix of Inertial Temperature Auction . . . . . . . . . . . . . . 71

3.8.1 Proof of concavity . . . . . . . . . . . . . . 71

3.8.2 Proof of Lemma 3.1 . . . . . . . . . . . . . . . . 72

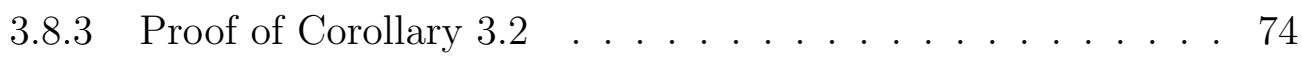

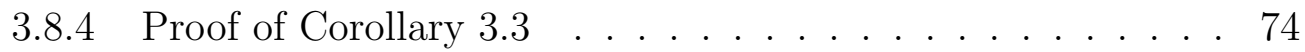

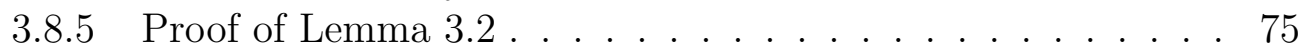

4 Truthful Multichannel Auction $\quad 77$

4.1 Introduction . . . . . . . . . . . . . . . . . . 77

4.1.1 Literature Review . . . . . . . . . . . . . . . . . . . . . 79

4.2 Mathematical Framework . . . . . . . . . . . . . . . . . 80

4.3 Truthful Multi Channel Auction (TMCA) . . . . . . . . . . . . . 84

4.3.1 Properties of TMCA . . . . . . . . . . . . . . 88

4.3.2 TMCA Optimal Reserve Price . . . . . . . . . . . . . . . . 89

4.4 Numerical Experiments and Analysis . . . . . . . . . . . . . . . 95

4.4 Low Density Mesh . . . . . . . . . . . . . . . . . 96

4.5 Conclusion . . . . . . . . . . . . . . . . . . . . . . . 101

4.6 Appendix . . . . . . . . . . . . . . . . . . . . . . . 104

4.6.1 Appendix: NP-Completeness of channel allocation . . . . . . 104

4.6.2 Appendix: Preallocation Phase and Feasibility . . . . . . . 105

4.7 Conclusions . . . . . . . . . . . . . . . 106

$\begin{array}{ll}\text { Bibliography } & 108\end{array}$ 


\section{Chapter 1}

\section{Introduction}

During the last two decades, the communication industry has experienced extraordinary growth. The prediction of a world where instantly transmitting a variety of data types to almost any device, anywhere in the world has been materializing before our eyes. This situation challenges regulators as well as service providers and has motivated increasing research in four fronts [15]: (i) development of new communication technologies, (ii) development of new services and applications, (iii) improved resource efficiency through market-based mechanisms, and (iv) understanding of economic and financial implications.

This work focuses on the advances on improved resource efficiency through market-based mechanisms, a topic in which the Federal Communications Commission (FCC) has put a great emphasis. In this matter, the communication community has naturally shifted its attention towards the field of mechanism design in order to find suitable market-based mechanisms. Mechanism design is a field in game theory used by economists in order to provide the right incentives on consumers to achieve a desired resource configuration (or set of consumption bundles). The FCC has a well known history with the field of mechanism design. Since the year 1994 the FCC has been using auctions to preform primary spectrum allocations where communication business receive long-term spectrum usage rights. For example, between 1994 and 2005 the FCC ran more than 70 auctions to allocate 
spectrum bands ${ }^{1}$.

By the year 2000 early signs of spectrum shortage motivated the FCC to update their guidance on spectrum management policies. Since then, the FCC has promoted secondary markets, where current spectrum holders benefit from trading their spectrum rights with secondary users in order to improve efficiency in the use of spectrum. According to the FCC [9]:

“... An effective way to make unused spectrum held by existing licensees available to others may be through secondary markets. An effectively functioning system of secondary markets would encourage licensees to be more spectrum efficient by freely trading their rights to unused spectrum capacity, ... We also believe that secondary market transactions could contribute to increasing the amount of spectrum available to prospective users, uses, and new wireless technologies by making more effective use of spectrum that is currently assigned to existing licensee ".

The last decade not only witnessed unprecedented growth in the demand for wireless bandwidth; similarly, the communication physical backbone infrastructure has also been pushed to its limits, validating the use of secondary markets into these technologies. Secondary markets introduce new dynamics that stem from the variety of simultaneous users accessing network resources. These new dynamics in turn introduce new difficulties as discussed later. The type of markets proposed by the FCC increase the complexity of the network management, since resources are now shared by a set of sophisticated and selfish users under some market rules. Section 1.0.1 explains how these conditions influence the design of new network protocols and the increasingly important role mechanism design should play in them.

This thesis uses the theory common to the field of mechanism design to solve problems of resource allocation in communication networks considering technological implications and difficulties that arise in the presence of sophisticated rational users and technological considerations. To do so, this thesis presents different

\footnotetext{
${ }^{1}$ see http://wireless.fcc.gov/auctions
} 
problems that pose a variety of difficulties and develops algorithms with some theoretical properties desired in both the theory of communication networks and the theory of mechanism design that overcome some of these difficulties.

\subsubsection{Mechanism Design in Communication Networks}

Communication networks' resources are generally shared by several users. How to effectively share these resources has been a topic of research for the last decades. While economists have long studied the means to achieve several properties in the allocation of resources, engineers in the communications community have designed network protocols focusing on technological challenges and some economic considerations have been often overlooked. For example, network users are usually assumed to behave according to well defined protocols that guarantee an effective use of resources. However for modern communication networks, generally constituted by heterogeneous and profit maximizing users, it is not clear why we should rely on such an assumption.

It is not evident that rational, profit maximizing, network users will cooperate to maximize the overall network performance at the expense of their individual benefits. In Jain's words: "Network capacity can be enhanced by sophisticated information-theoretic and network coding methods, but those will not be enough without incentivizing cooperation between increasingly sophisticated and selfish users" [16].

In the field of communication networks, it has been a common practice to study the optimal use of resources as convex optimization problems. Users are assumed to willingly follow predefined rules, which in turn guarantee the implementation of an "optimal" configuration of the resources [21,23]. These works, however, are prone to manipulation by strategic and sophisticated users, hence the need to consider incentives in the design of algorithms related to the allocation of network resources. In light of this, network theorists have looked onto the field of mechanism design for the right incentives to achieve a desired resource configuration. 
Despite the advances in the field of mechanism design, off the shelf results are not always directly applicable to the field of communication networks. Many advances in mechanism design theory assume each consumer is only affected by his own consumption bundle $[1,2,16,17,20]$. Usually utility of consumer $i$ is modeled by a concave function $U_{i}\left(\mathbf{x}_{i}\right)$ where $\mathbf{x}_{i}$ is the consumption bundle of consumer $i$. In contrast with this model, some communication problems are subject to externalities in the sense that the utility of one user does not only depend on his own resource consumption bundle but on his competitors as well. Such externalities are usually negative (i.e. the utility of any user is eroded by the consumption of his competitors). Furthermore the way such externalities are considered drastically change depending upon technological considerations. For example, chapter 3 of this thesis introduces an uplink access problem using Code Division Multiple Access (CDMA) technology, where all users associated with an access point (AP) share the same bandwidth and the rate of each user is increasingly eroded as the power of other users increases (in a continuous fashion). In contrast, chapter 4 introduces a Frequency Division Multiple Access (FDMA) system where spectrum is partitioned into channels and later assigned to several potential users. In this problem, the use of one channel by any user precludes its use by a subset of other users; note these externalities are binary instead of continuous.

Before bringing forward the contributions of this work, it is important to introduce some basic concepts of the theory of mechanism design in the following section.

\subsubsection{A Brief Introduction to the Theory of Mechanism Design}

Mechanism design is a field of game theory in which rules (mechanisms) are designed in order to induce a desired equilibrium outcome resulting from the interaction of rational agents with private information. In general, the designer wants to induce an outcome that holds certain properties related to the private information of these agents. For example, in the context of communication networks, such a 
desired outcome can be the efficient configuration or allocation of a resource (power, bandwidth, etc.) to set of rational users. Because such efficient configuration depends upon the private information of users, the resource manager can not find the desired outcome directly. Instead he designs a set of rules, such that the desired outcome is achieved by the strategic interaction of these rational agents.

For example, consider a set of transmitter-receiver (tx-rx) pairs with a quasilinear utility (i.e. willingness to pay) for any network configuration and spectrum bandwidth allocation. Under a given network configuration and bandwidth allocation, different transmitter-receiver pairs may experience different utility, and this information is not necessarily known by other parties. A benevolent resource manager may implement a mechanism design in which he asks each tx-rx pair to reveal its preferences and implement the outcome that maximizes the aggregate utility. However this mechanism is naive because it does not consider strategic players who may lie to increase their individual utilities at the expense of others. To avoid this situation the designer needs to enforce some payment rules. When these rules define a form of competition among agents, such as network users, the mechanism is called an auction [22], which is the type of mechanism considered in this work.

The mechanism design approach is composed of two elements, the environment and the mechanism [25].

The environment $\left(\mathcal{N},\left\{t_{i}\right\}_{i \in \mathcal{N}}, \mathcal{X}\right)$ is composed of:

- A set of agents or participants, $\mathcal{N}=\{1, \ldots, N\}$

- A set of possible outcomes $\mathcal{X}$.

- Each agent $i \in\{1, \ldots, N\}$ has a type $t_{i}$, which is related to $i$ 's preferences and beliefs.

The mechanism $\left(\left\{S_{i}\right\}_{i \in \mathcal{N}}, x(\cdot),\left\{p_{i}(\cdot)\right\}_{i \in \mathcal{N}}\right)$ consists of rules that govern: 
- What the participants are allowed to do $\left(\left\{S_{i}\right\}_{i \in \mathcal{N}}\right)$. This is also known as the strategy space of the agents.

- How participants' actions determine the outcome. That is, a function $x(\cdot)$ : $S_{1} \times \cdots \times S_{N} \rightarrow \mathcal{X}$.

- How participants' actions determine their payments. That is, a set of functions $\left\{p_{i}(\cdot): S_{1} \times \cdots \times S_{N} \rightarrow R_{+}\right\}_{i \in \mathcal{N}}$.

A very common assumption, adopted in this work, is that utilities are quasilinear and private in the sense that for any outcome $x \in \mathcal{X}$ and payment $p_{n} \in \mathbb{R}$, profit of agent $n \in \mathcal{N}$ is of the form $\pi_{n}\left(x, p_{n}, t_{n}\right)=u_{n}(x)-p_{n}$ where $u_{n}(x) \in \mathbb{R}$ is the intrinsic utility agent $n \in \mathcal{N}$ derives from outcome $x \in \mathcal{X}$. Note that $u_{n}()$ pertains to $n$ 's private information $t_{n}$.

Fig.1.1 shows how mechanism design works. In this figure each agent $n \in$ $\{1, \ldots, N\}$, has a privately known type $t_{n}$ and performs an action $s_{n}$. The resource manager applies some rules (known as mechanism) that map each possible action profile $\left(s_{1}, \ldots, s_{N}\right)$ to an output $x\left(s_{1}, \ldots, s_{N}\right)$ and a set of payments $\left\{p_{i}\left(s_{1}, \ldots, s_{N}\right)\right\}_{i \in \mathcal{N}}$. This field is called mechanism design because it studies how to design these set of rules looking forward to achieve certain properties which are discussed later in this chapter. Agents are assumed to be profit maximizers. That is, if $T_{n}$ is the set of possible types for agent $n \in \mathcal{N}$, he will use a strategy $\sigma_{i}(\cdot): T_{i} \rightarrow S_{i}$, in order to maximize his own profit $\pi_{n}$.

A simple illustration helps clarify this point. Consider a set $\mathcal{N}=\{1,2\}$ of wireless network operators (rational agents) that compete to receive one channel (resource). The set possible outcomes is $\mathcal{X}=\{[1,0],[0,1]\}$, where outcome $[1,0]$ represents network 1 receiving the channel and $[0,1]$ network 2. Private information $\left\{t_{n}: n \in \mathcal{N}\right\}$ is the valuation each network attains when receiving the channel, say $v_{1}=10$ for network 1 and $v_{2}=5$ for network 2 (or what is the same $u_{1}([1,0])=10$, 


\section{Agents Strategies}

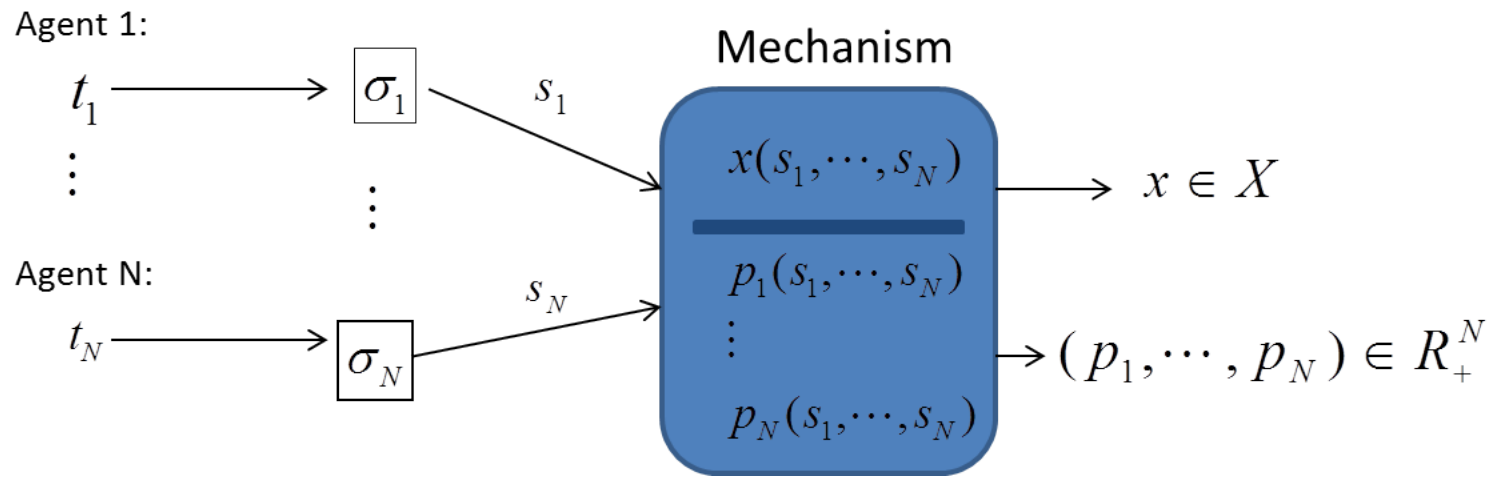

Figure 1.1: Mechanism design elements (Environment and Mechanism)

$\left.u_{2}([0,1])=5, u_{1}([0,1])=u_{2}([1,0])=0\right)$. The auctioneer wants to allocate the channel efficiently but ignores the utility functions of both competitors.

In this simple scenario the auctioneer can implement a second price auction. In this auction each network is asked to report its private valuation. Each bidder $n \in \mathcal{N}$ can report any non-negative value, hence $S_{n}=\mathbb{R}_{+}$. Let $\sigma_{n}()$ denote the strategy of network $n$ 's operator and let $s_{n}=\sigma_{n}\left(v_{n}\right)$ be his reported valuation. In the second price auction the channel is allocated to the bidder with highest reported valuation as follows:

$$
x\left(s_{1}, s_{2}\right)= \begin{cases}{[1,0]} & \text { if } s_{1}>s_{2} \\ {[0,1]} & \text { otherwise }\end{cases}
$$

The winner is assessed a payment equal to his competitors reported valuation. That is let $m \neq n$, then:

$$
p_{n}\left(s_{1}, s_{2}\right)=\left\{\begin{array}{cc}
s_{m} & \text { if } s_{m}<s_{n} \\
0 & \text { otherwise }
\end{array}\right.
$$

It is a well known result that for this auction, being truthful (i.e. setting $\left.s_{n}=v_{n}\right)$ is a dominant strategy, that is, let $m \neq n$, then:

$$
\pi_{n}\left(x\left(s_{n}, s_{m}\right), p_{n}\left(s_{n}, s_{m}\right), t_{n}\right) \leq \pi_{n}\left(x\left(v_{n}, s_{m}\right), p_{n}\left(v_{n}, s_{m}\right), t_{n}\right)
$$


for all $n \in \mathcal{N}$, and $s_{n}, s_{m} \in \mathbb{R}_{+}$. Furthermore note that when users are truthful, the auctioneer implements the efficient allocation. In light of this we say that the auctioneer implements the efficient allocation in dominant strategies.

The resource manager (auctioneer) may have different objectives in his design. Consider for example the case where the auctioneer is a public entity that looks after the use of some public resources. In this case efficiency is a primary objective. On the other hand, consider the case of a private entity who wants to accrue revenue from auctioning idle resources. In this case revenue may probably get a primary role over efficiency. This thesis focuses on efficiency.

Some characteristics that are usually mentioned in a mechanism design are:

- Direct Mechanism: We say a mechanism is direct if bidders are directly asked to reveal their private information, that is $S_{n}=T_{n}$ for all $n \in \mathcal{N}$.

- Incentive Compatibility: We say a mechanism is incentive compatible (IC) if each agent $n \in \mathcal{N}$ has incentives to reveal the information required by the auctioneer (in a direct mechanism this means to make $s_{n}=t_{n}$ ). Note that incentive compatibility may be achieved in different degrees (Nash Equilibrium / Dominant strategies).

- Individual Rationality: We say a mechanism is individually rational if all agents are willing to participate. That is, the profit an agent can expect from participation is non-negative.

- Revenue Maximizing: We say that a mechanism is Revenue Maximizing if it maximizes the expected revenue of the mechanism designer (resource manager).

- Efficiency: We say that a mechanism is efficient if the outcome (resource allocation in our problems) maximizes the aggregate social surplus.

If a mechanism is incentive compatible in dominant strategies and it is efficient, this mechanism is said to truthfully implement the efficient outcome in dominant strategies. 
These properties are always desired. Unfortunately, designing a mechanism that is simultaneously IC, revenue maximizing, and efficient has proven impossible $[12,33]$.

\subsubsection{Contributions of this Thesis}

This thesis presents three problems related to the allocation of communication resources. For each of these problems we propose an auction that is Incentive Compatible in Dominant Strategies. Note this is stronger than the more common condition of Nash Equilibria. That is, in the auction designs we propose, being truthful is in the best interest of each bidder, even if his competitors are not truthful.

Chapter 2 introduces the problem of a wired-network manager that wants to efficiently allocate network capacity amongst a set of potential users with private information about their utilities. The network exhibits a congestion externality in the sense that the utility a given user derives from using a given link in the network is decreasing in the aggregate intensity with which other users make use of that same link. Although the well known VCG mechanism can be used in this context, several drawbacks have been documented by theorists in both auction design and communication networks $([3,30,35])$, which have been mainly related to confidentiality issues and the need for sophisticated computational infrastructure. Iterative auction designs have been proposed in order to overcome these problems. However, iterative auctions in literature do not account for congestion externalities. Chapter 2 proposes an iterative auction, which to the best of my knowledge, is the first strategy-proof iterative auction that implements the efficient allocation of capacity in a network subject to congestion externalities.

Wireless communication resources are also subject to negative externalities. In fact, due to the flexibility provided by different wireless access schemes, there exist a rich variety of considerations when modeling these externalities. The increasing use of wireless devices and applications has prompted the search for mechanisms 
to dynamically and efficiently reallocate relatively underutilized licensed spectrum. Such mechanisms would enable a secondary market in which unlicensed users can obtain the resources they need, and licensed users are incentivized to offer resources that are not utilized. This secondary market poses special challenges especially because such allocations need to consider technical issues (e.g. interference) as well as transparency and economic efficiency. This market is unique in that spectrum can be reused; the same spectrum can be simultaneously employed by various users without precluding each other's use. Chapters 3 and 4 address wireless spectrum allocation under different technological considerations. Chapter 3 addresses the allocation of some residual power in a set of APs for the case of uplink using Code Division Multiple Access (CDMA). Chapter 4 introduces the problem of designing a mechanism to deal with spectrum allocation when using Frequency Division Multiple Access (FDMA).

Chapter 3 considers the problem of a primary UL-network where a set of APs can tolerate some additional interference without compromising the service of some intrinsically associated users. The network manager wants to efficiently allocate these levels of interference (usually known as interference temperature) to a set of secondary users with private information about their utilities. As stated before, the design depends upon the access method. In Chapter 3 we assume Code Division Multiple Access, Direct Sequence Spread Spectrum (CDMA-DSSS) in which each user is assigned a code that allows him to share the same bandwidth with several other users. Of course, users are subject to externalities; the rate any user achieves with any AP will be eroded by the power of his competitors at such AP. In this problem, VCG also provides many desired properties, yet concerns related to confidentiality and computational complexity arise. Iterative auctions have been proposed to overcome these issues $[5,14,31]$. However, these auction designs can only guarantee efficiency under restrictive conditions, where either, specific utility functions are required, or power of secondary users is assumed negligible compared to that of the primary users. Chapter 3 proposes an auction design that to the 
best of our knowledge is the first truthful, iterative auction that achieves efficiency in dominant strategies without very restrictive assumptions.

Chapter 4 addresses spectrum sharing in Frequency Division Multiple Access (FMDA), that is, when the spectrum has been partitioned into channels of semiexclusive use. It studies the problem of a channel broker who has a set of channels he wants to allocate to a set of secondary users. In this context, a single channel can be allocated to several users as long as the interference between them is kept between acceptable levels. These types of restrictions are considered by means of a conflict graph that implicitly define feasible channel allocations. This problem was initially introduced by Zhou [37], and has been studied by several authors since then $[4,19,34,38]$. These works have proposed incentive compatible auctions that assume fixed marginal utility from bidders and which are proved to be implementable in polynomial time. Chapter 4 of this thesis introduces Truthful Multichannel Auction (TMCA), a channel auction that to the best of my knowledge is the first one-shot channel auction that is incentive compatible in dominant strategies and implementable in polynomial time when marginal utilities of bidders are not fixed, which is a more realistic assumption. 


\section{Chapter 2}

\section{Inertial Capacity-Auction}

\section{$2.1 \quad$ Preliminaries}

\subsubsection{Introduction}

In this chapter, we consider the problem faced by a network manager whose goal is to efficiently allocate network capacity amongst a finite set of non-cooperative users that have private information regarding their utility. The network exhibits a congestion externality in the sense that the utility a given user derives from using a given link in the network is decreasing in the aggregate intensity with which other users make use of that same link. The network is operated with fixed routing tables, and users autonomously determine their desired flow rates.

For this problem we propose the Inertial Capacity Auction (ICA), an iterative auction in which, network users are incentivized to repeatedly update their use of the network, according to some predefined rules. This updates, in turn, guarantee the efficient allocation of network capacity.

\subsubsection{Basic Framework}

Consider a network $(\mathcal{V}, \mathcal{E})$ where $\mathcal{V}=\{1, \ldots, V\}$ represents the index set of vertices and $\mathcal{E}=\{1, \ldots, E\}$ the index set of links. Let $\bar{\lambda}_{e}>0$ denote the capacity of link $e \in \mathcal{E}$ and let $\overline{\boldsymbol{\lambda}}=\left[\bar{\lambda}_{1}, \ldots, \bar{\lambda}_{E}\right]$ denote the joint capacity of the network. This network is managed by a benevolent manager who aims at optimizing the net 
aggregate utility of a set of users $\mathcal{M}=\{1, \ldots, M\}$. Each user $m \in \mathcal{M}$ is associated with an origin-destination pair $\left(o_{m}, d_{m}\right) \in \mathcal{V} \times \mathcal{V}$ and has a fixed routing policy such that its use of link $e \in \mathcal{E}$ per flow unit is $A_{m, e} \in[0,1]$.

Let $r_{m}$ denote the flow of user $m \in \mathcal{M}$, and $\boldsymbol{r}=\left[r_{1}, \ldots, r_{M}\right]$ denote the joint flow profile. The aggregate flow on link $e \in \mathcal{E}$ is

$$
\lambda_{e}(\boldsymbol{r})=\sum_{m \in \mathcal{M}} A_{m, e} r_{m}
$$

Let $U_{m}(): \mathbb{R}_{+} \rightarrow \mathbb{R}_{+}$be a strictly concave function, privately known by user $m \in \mathcal{M}$, such that $U_{m}\left(r_{m}\right)$ dictates the intrinsic utility of user $m \in \mathcal{M}$ given flow $r_{m} \in \mathbb{R}_{+}$. We assume congestion externalities are additive so that $m$ 's quasilinear utility (i.e. willingness to pay), given joint profile $\boldsymbol{r}=\left[r_{1}, \ldots, r_{M}\right]$, is of the form:

$$
U_{m}\left(r_{m}\right)-r_{m} \sum_{e \in \mathcal{E}} A_{m, e} c_{e}\left(\lambda_{e}(\boldsymbol{r})\right)
$$

where $c_{e}(\cdot)$ is an increasing, concave and twice continuously differentiable function describing the per-unit congestion disutility affecting users of link $e \in \mathcal{E}$.

It follows that the aggregate utility of all users is:

$$
\sum_{m \in \mathcal{M}}\left[U_{m}\left(r_{m}\right)-r_{m} \sum_{e \in \mathcal{E}} A_{m, e} c_{e}\left(\lambda_{e}(\boldsymbol{r})\right)\right]=\sum_{m \in \mathcal{M}} U_{m}\left(r_{m}\right)-\sum_{e \in \mathcal{E}} \lambda_{e}(\mathbf{r}) c_{e}\left(\lambda_{e}(\mathbf{r})\right)
$$

The benevolent network manager wants to implement the efficient flow profile, that is, the joint flow profile $\mathbf{r} \in \mathbb{R}_{+}^{M}$ that solves the following optimization problem:

$$
\begin{array}{ll}
\max _{\mathbf{r} \in \mathbb{R}_{+}^{M}} & \sum_{m \in \mathcal{M}} U_{m}\left(r_{m}\right)-\sum_{e \in \mathcal{E}} \lambda_{e}(\mathbf{r}) c_{e}\left(\lambda_{e}(\mathbf{r})\right) \\
\text { s.t. } & \\
& \lambda_{e}(\mathbf{r}) \leq \bar{\lambda}_{e} \quad e \in \mathcal{E}
\end{array}
$$

Since intrinsic utility functions $\left\{U_{m}: m \in \mathcal{M}\right\}$ are private information, only known by the respective users, the network manager can not find the desired outcome directly. In this chapter we propose Inertial Capacity Auction (ICA), an auction 
mechanism by which the network manager can implement the efficient configuration in dominant strategies. In order to provide some intuition in the rules proposed in ICA, let us make some important observations.

From strict concavity and differentiability, sufficient conditions for optimality of a joint flow profile $\mathbf{r}=\left[r_{1}, \ldots, r_{M}\right]$ are:

$$
\begin{gathered}
U_{m}^{\prime}\left(r_{m}\right)-\sum_{e \in \mathcal{E}} A_{m, e}\left[c_{e}\left(\lambda_{e}(\mathbf{r})\right)+\lambda_{e}(\mathbf{r}) c_{e}^{\prime}\left(\lambda_{e}(\mathbf{r})\right)+\mu_{e}\right] \leq 0 \quad \text { for all } m \in \mathcal{M} \\
\text { with equality if } r_{m}>0
\end{gathered}
$$

and

$$
\mu_{e}\left(\bar{\lambda}_{e}-\lambda_{e}(\mathbf{r})\right)=0 \quad \text { for all } e \in \mathcal{E}
$$

where $\mu_{e} \geq 0$ for all $e \in \mathcal{E}$ and $r_{m} \geq 0$ for all $m \in \mathcal{M}$. Furthermore, strict concavity implies the efficient flow profile is unique.

Assume the hypothetical situation in which the network manager knows the efficient joint flow profile, say $\mathbf{r}^{*} \in \mathbb{R}_{+}^{M}$ and shadow prices $\left\{\mu_{e} \geq 0: e \in \mathcal{E}\right\}$. In this case, the network manager can set a game in which users are guaranteed a per flow-unit cost on link $e \in \mathcal{E}$ of $p_{e}=\mu_{e}+\lambda_{e}\left(\mathbf{r}^{*}\right) c_{e}^{\prime}\left(\lambda_{e}\left(\mathbf{r}^{*}\right)\right)+c_{e}\left(\lambda_{e}\left(\mathbf{r}^{*}\right)\right)$, no matter the resultant flow configuration. Then user $m \in \mathcal{M}$ faces the following problem:

$$
\max _{r_{m} \in \mathbb{R}_{+}}\left[U_{m}\left(r_{m}\right)-r_{m} \sum_{e \in \mathcal{E}} A_{m, e}\left(\mu_{e}+\lambda_{e}\left(\mathbf{r}^{*}\right) c_{e}^{\prime}\left(\lambda_{e}\left(\mathbf{r}^{*}\right)\right)+c_{e}\left(\lambda_{e}\left(\mathbf{r}^{*}\right)\right)\right)\right]
$$

If users are rational, they will respond with the efficient flow profile. To see this, note that by concavity, user $m \in \mathcal{M}$ will set his flow to $r_{m} \in \mathbb{R}_{+}$such that

$$
U_{m}^{\prime}\left(r_{m}\right)-\sum_{e \in \mathcal{E}} A_{m, e}\left[\mu_{e}+\lambda_{e}\left(\mathbf{r}^{*}\right) c_{e}^{\prime}\left(\lambda_{e}\left(\mathbf{r}^{*}\right)\right)+c_{e}\left(\lambda_{e}\left(\mathbf{r}^{*}\right)\right)\right] \leq 0
$$


with equality if $r_{m}>0$. Note that because of the strict concavity of $U_{m}$, condition in Eq. (2.3) uniquely defines $r_{m}$. Furthermore, this condition resembles condition in Eq. (2.2.a).

The auction design we propose in the following sections runs in discrete time steps (or rounds) indexed by $t \in\{0,1,2, \ldots\}$. At each round a price for unit flow on each link $e \in \mathcal{E}$ is set, and secondary users are asked to update their flows following a predefined rule (or strategy) that involves some of their private information; users that follow this rule are called truthful. Given the private information involved, users may not be truthful and respond to this request following their own strategy. However, the maximum benefit any user can attain by not being truthful can be made as small as desired, no matter the strategy followed by his competitors. In other words, being truthful is an $\epsilon$-dominant strategy. Furthermore, when all users are truthful, the socially efficient configuration is implemented. In the context of mechanism design theory, ICA is said to truthfully implement the socially efficient outcome in $\epsilon$-dominant strategies.

\subsection{Literature Review}

In this section, we review the relevant literature by using a simple illustrative example wherein a single, congestible link with unit capacity is shared by two

users. Each user $m \in\{1,2\}$ can determine his own flow on the link, say $r_{m} \geq 0$, and has privately known intrinsic utility $U_{m}\left(r_{m}\right)=\ln \left(1+r_{m}\right)$. At this point we do not account for externalities as these are not considered in the earliest pieces of literature we review.

There is a vast amount of literature on congestion pricing as a means to optimizing network flows $([20,21,23])$. In most of these works, the network manager sets congestion prices, and network users respond by adjusting their flows accordingly. Typically, prices are adjusted so as to reflect excess demand for limited resources and users react to these prices by adjusting their desired flow rates. However under incomplete information assumptions, strategic users 
can manipulate congestion prices to their individual advantage by not truthfully reporting their optimal flow rates. In turn, this strategic behavior distorts the intended optimality of the network configuration (see for example the works of Jang and Hajek [35]).

Consider, for example, the simple iterative pricing mechanism proposed by Kelly [21], which is sometimes referred to as the Kelly mechanism. Let $r_{1}^{t}$ and $r_{2}^{t}$ denote the flow rates at time $t>0$. The Kelly mechanism assumes users are price-takers. That is, it assumes that at each time $t \in\{0,1,2, \ldots\}$, flows $r_{1}^{t}$ and $r_{2}^{t}$ are updated in response to current prices disregarding possible changes in such prices $[21,35]$. The Kelly mechanism induces the efficient flow configuration in the limit (i.e. $r_{m}^{t} \rightarrow r_{m}^{*}=0.5, m \in\{1,2\}$ ) at a price (per unit of flow) of $\mu^{*}=\frac{2}{3}$. Note that, in this case, profit of user 1 is $\Pi_{1}^{*}=\ln \left(1+r_{1}^{*}\right)-\mu^{*} r_{1}^{*}$.

Unfortunately this mechanism is not strategy-proof. If user 1 deviates from the price taking behavior, and updates $r_{1}^{t}$ as if his utility function were $\widetilde{U}_{1}\left(r_{1}\right)=$ $0.8 \ln \left(1+r_{1}\right)$, the Kelly mechanism would lead to a final allocation $\left(\tilde{r}_{1}, \tilde{r}_{2}\right)=\left(\frac{1}{3}, \frac{2}{3}\right)$ with a price of $\widetilde{\mu}=0.6$. In this case, user 1's profit is $\widetilde{\Pi}_{1}=\ln \left(1+\widetilde{r}_{1}\right)-\widetilde{\mu} \widetilde{r}_{1}$, which exceeds the profit associated with a price taking behavior, namely $\Pi_{1}^{*}$. Intuitively, user 1 is able to induce lower prices at the expense of reduced flow rate, yielding an overall positive effect in net profit.

The theory of mechanism design provides guidance on incentive structures that eliminate the adverse effects of strategic behavior. For example, the celebrated Vickrey-Clarke-Groves (VCG) mechanism $[8,13,32]$ is a direct mechanism that is strategy proof and implements the socially efficient outcome (flow configuration in our case). In VCG, users report their intrinsic utility functions, the efficient capacity allocation (based upon the reported utility functions) is implemented and each user is charged the "opportunity cost" that his participation generates to his competitors. The opportunity cost incurred by a user corresponds to the difference in his competitors' aggregate utility when he does not participate and when he does. 
In our example, assuming users are truthful, VCG implements flows $\left(r_{1}^{*}, r_{2}^{*}\right)=$ $(0.5,0.5)$ since these maximize the social utility $U_{1}\left(r_{1}\right)+U_{2}\left(r_{2}\right)$. Note that if user $m$ had not participated, user $j \neq m$ would have been allocated a flow of $r_{j}=1$, instead of $r_{j}^{*}=0.5$. Hence, $m$ 's payment, which correspond to the opportunity cost $m$ 's participation introduces to his competitor $j$, is $P_{m}=U_{j}(1)-U_{j}\left(r_{j}^{*}\right)=0.288$ where $j \in\{1,2\}$ and $j \neq m$. Hence, user $m$ 's profit is $\Pi_{m}^{*}=U_{m}\left(r_{m}^{*}\right)-P_{m}=$ $U_{m}\left(r_{k}^{*}\right)+U_{m}\left(r_{j}^{*}\right)-U_{m}(1)=0.118$.

Consider now the case where user $m \in\{1,2\}$ does not report his utility function truthfully (for example by reporting $\widetilde{U}_{m}\left(r_{m}\right)=0.8 \ln \left(1+r_{m}\right)$ ). In this case, the VCG mechanism would implement some allocation $\left(\widetilde{r}_{1}, \widetilde{r}_{2}\right)$ with a net profit for $m$ of $\widetilde{\Pi}_{m}=U_{m}\left(\widetilde{r}_{m}\right)+U_{j}\left(\widetilde{r}_{j}\right)-U_{j}(1)$ which by optimality of $\left(r_{1}^{*}, r_{2}^{*}\right)$ is not greater than $\Pi_{m}^{*}$.

Despite its desirable properties, mainly incentive compatibility and efficiency, the VCG mechanism has many practical drawbacks $[3,27,30]$. Probably the main drawback of VCG is that users are required to fully reveal their utility structure, which conveys highly sensitive information. A second drawback pertains to computational burden, because finding the optimal network flow (given the utility functions reported by users) may require a non-negligible computational infrastructure.

Theorists have long expressed some concerns about direct mechanisms (also known as direct-revelation mechanisms) such as VCG. In a direct mechanism, bidders are asked to fully reveal their private information which places high demands on information revelation. In light of this concern, theorists have looked onto the design of iterative mechanisms. In Parkes's words [27]: " Direct revelation mechanisms are often too expensive for agents because they place very high demands on information revelation. An iterative mechanism can sometimes implement the same outcome as a direct-revelation mechanism but with less information revelation and agent computation".

In an effort to combine the efficiency and incentive compatibility properties of 
VCG with the iterative nature of the Kelly mechanism, which helps circumvent the main drawbacks of VCG, Yang and Hajek introduced the VCG-Kelly mechanism [35]. As with the VCG, the VCG-Kelly mechanism also implements the socially efficient outcome albeit in a weaker form of implementation (Nash equilibrium implementation). A closely related work is presented by Jain and Walrand [17]. It is worth noting that not Yang and Hayek [35] nor Jain and Walrand [17] take into consideration congestion externalities.

In a separate and more general approach, Ausubel has recently proposed relatively simple iterative auction designs that circumvent many of the practical difficulties associated with the VCG mechanism [1,2]. Ausubel's designs propose an ingenious payment rule by which the efficient allocation of commodities (possibly, heterogeneous and discrete) are truthfully implemented in Nash equilibrium. Ausubel's auction designs parallel a primal-dual algorithm where the dual solution yields the market clearing prices (for details, see the works of Bikhchandani and Ostroy [6]). In these designs, at every iteration, users are asked to report their desired demand for the available commodities, given a set of prices. Thus, users only need to partially reveal their utility structures (in contrast to the VCG mechanism). Ausubel's designs also have low computational requirements. Prices are updated from one round to the next according to relatively simple rules reflecting excess demand. Thus, there is no need to solve large-scale optimization problems.

Ausubel's auction design can be applied to network flows optimization, however it may fail to clear demand when there are congestion externalities. To illustrate this point, consider again the problem of the link with unit capacity and two users with intrinsic utilities $U_{m}\left(r_{m}\right)=\ln \left(1+r_{m}\right)$ for $m \in\{1,2\}$. This time assume an additive externality as described in section 2.1 .2 where congestion cost is $c(\lambda)=\frac{2}{3} \lambda^{2}$, so that net utility of user $m \in\{1,2\}$ is $\ln \left(1+r_{m}\right)-r_{m} \frac{2}{3}\left(r_{1}+r_{2}\right)^{2}$. A discrete-time implementation of Ausubel's design could be as follows. Let $\mu^{t}$ denote the price for unit flow on the link and $r_{m}^{t} \in \mathbb{R}_{+}$denote the flow of user 
$m \in\{1,2\}$ at time $t \in\{0,1,2, \ldots\}$. We say user $k$ updates his rate truthfully if:

$$
r_{m}^{t+1}=\arg \max _{r \in \mathbb{R}_{+}}\left[U_{m}(r)-r\left(c\left(\lambda^{t}\right)+\mu^{t}\right)\right]
$$

where $\lambda^{t}=r_{1}^{t}+r_{2}^{t}$. At each time $t>0$ prices are updated as follows:

$$
\mu^{t+1}=\left[\mu^{t}+\rho_{t}\left(r_{1}^{t+1}+r_{2}^{t+1}-1\right)\right]^{+}
$$

where $\rho_{t}=\frac{1}{t+1}$ and $[x]^{+}=\max \{x, 0\}$.

Assuming users update their flow truthfully, this implementation induces the cyclical behavior in Figure 2.1.

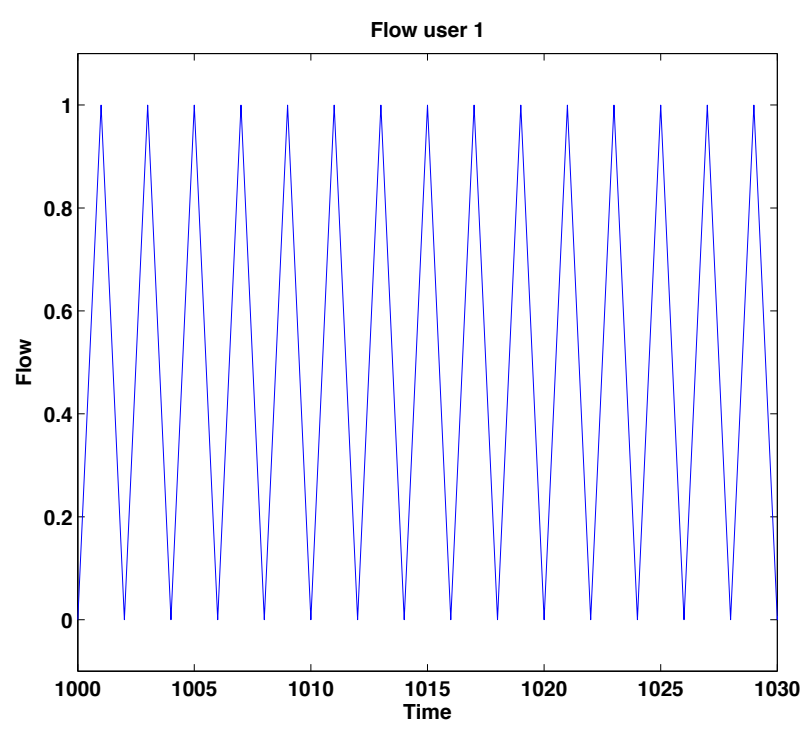

Figure 2.1: Flow demand of user 1

Note that Eq. 2.3 suggests that an externality price of $\lambda^{t} c^{\prime}\left(\lambda^{t}\right)$ may be assessed with the goal of inducing an efficient flow allocation. In this case we say that users update truthfully if:

$$
r_{m}^{t+1}=\arg \max _{r \in \mathbb{R}_{+}}\left[U_{m}(r)-r\left(c\left(\lambda^{t}\right)+\mu^{t}+\lambda^{t} c^{\prime}\left(\lambda^{t}\right)\right)\right]
$$

Unfortunately the use of this externality price does not guarantee flow convergence. When implementing this externality price in or example above and assuming 
truthful users, the outcome is essentially the same as in Fig. 2.1.

In this chapter, we propose Inertial Capacity Auction (ICA), an iterative auction design that implements (in $\epsilon$-dominant strategies) the socially efficient flow allocation in a network prone to congestion externalities. Our design is relatively simple. At each time period, users set demand as a response to previous prices and congestion conditions. However, flows are not immediately updated to such demand; they are updated towards demand with an increasing inertia that prevents abrupt changes in the network flow configuration. This feature introduces an element of inertia that ultimately allows for market clearing. To the best of our knowledge, ours is the first strategy-proof iterative mechanism that implements the efficient allocation of capacity in a network subject to congestion externalities.

This chapter is organized as follows; in Section 2.3, we propose ICA, Section 2.4, introduces some basic assumptions, and Sections 2.5 and 2.6 prove that ICA truthfully implements the efficient flow configuration in $\epsilon$-Dominant strategies. Finally, Section 2.7 presents some conclusions and important remarks.

\subsection{Inertial Capacity Auction (ICA)}

In this section we formally introduce the Inertial Capacity Auction (ICA) for the efficient flow configuration in a capacity-constrained network. This auction runs in discrete time steps (or iterations) indexed by $t \in\{1,2, \ldots\}$. At each iteration the network manager reveals a congestion control price $\mu_{e}^{t}$ and an externality price $\zeta_{e}^{t}$ for each link $e \in \mathcal{E}$. Congestion control prices $\left\{\mu_{e}^{t}\right\}_{t \geq 0}$ will guarantee that the aggregate flow on link $e \in \mathcal{E}$ is less than or equal to its capacity $\bar{\lambda}_{e}$. Externality prices $\left\{\zeta_{e}^{t}\right\}_{t \geq 0}$ will help each users internalize the effect his individual flow has on his competitors.

The per flow-unit price on link $e \in \mathcal{E}$ at time $t \geq 0$, is the sum of the control price $\mu_{e}^{t} \in \mathbb{R}_{+}$and a externality price $\zeta_{e}^{t}$. Additionally, users of link $e \in \mathcal{E}$ suffer a per flow-unit cost of $c_{e}^{t}=c_{e}\left(\lambda_{e}\left(\mathbf{r}^{t}\right)\right)$. Hence the total (per flow-unit) cost experienced 
on link $e \in \mathcal{E}$ after time $t$ is $\left(c_{e}^{t}+\zeta_{e}^{t}+\mu_{e}^{t}\right)$. Given this costs, each user $m \in \mathcal{M}$ calculates his new flow demand $d_{m}^{t+1} \in \mathbb{R}_{+}$following a predefined rule (see Eq. 2.4), however flows are not immediately set to such demand, instead they are updated towards demand with an increasing inertia that prevents abrupt changes in the actual flow $r_{m}^{t}$.

Note that for any time $t \in\{1,2, \ldots\}$ we discriminate between $m$ 's demand $d_{m}^{t}$ and flow $r_{m}^{t}$. Similarly, joint demand profile at time $t>0$ is denoted by $\mathbf{d}^{t}=\left[d_{1}^{t}, \ldots, d_{M}^{t}\right]$ while joint flow profile is denoted by $\mathbf{r}^{t}=\left[r_{1}^{t}, \ldots, r_{M}^{t}\right]$. The relation between these two profiles is introduced below. In response to flow profile updates, the network manager updates the control prices profile $\boldsymbol{\mu}^{t}=\left[\mu_{1}^{t}, \ldots, \mu_{E}^{t}\right]$ and externality prices profile $\boldsymbol{\zeta}^{t}=\left\{\zeta_{1}^{t}, \ldots, \zeta_{E}^{t}\right\}$, which in turn triggers new demand and flow profiles. The process is repeated until the stationarity criteria $\left\|\mathbf{d}^{t+1}-\mathbf{r}^{t}\right\|+\left\|\boldsymbol{\mu}^{t}-\boldsymbol{\mu}^{t-1}\right\| \leq \eta$ is achieved for some small $\eta>0$. The following pseudo-code formally introduces Inertial Capacity Auction.

We assume $r_{m}^{0}=d_{m}^{0}=0, \zeta_{e}^{0}=0$ and $\mu_{e}^{0}=\bar{\mu}$ for all $m \in \mathcal{M}$ and $e \in \mathcal{E}$, where $\bar{\mu}$ is a price large enough to guarantee null demand from all users $m \in \mathcal{M}$ (more details on $\bar{\mu}$ in section 2.4). At each iteration $t>0$ the following 4 steps are executed

1. Demands Update: Given prices and congestion levels at the beginning of iteration $t$, each user $m \in \mathcal{M}$ calculates his flow demand $d_{m}^{t} \in \mathbb{R}_{+}$as follows:

$$
d_{m}^{t}=\underset{d \geq 0}{\operatorname{argmax}}\left[U_{m}(d)-d p_{m}^{t}\right]
$$

where $p_{m}^{t}$ is the total (per flow-unit) cost experienced by user $m \in \mathcal{M}$ at the beginning of iteration $t$ (i.e. $\left.p_{m}^{t}=\sum_{e \in \mathcal{E}} A_{m, e}\left(c_{e}^{t-1}+\zeta_{e}^{t-1}+\mu_{e}^{t-1}\right)\right)$. In what follows we shall refer to the demand in Eq.2.4 as the truthful demand of user $m$ at time $t$. 
2. Flows Update: Flows are updated as follows

$$
r_{m}^{t}=r_{m}^{t-1}+\alpha_{t}\left(d_{m}^{t}-r_{m}^{t-1}\right)
$$

for each $m \in \mathcal{M}$ where $\alpha_{t} \in(0,1)$ and $\alpha_{t} \rightarrow 0^{+}$(more details on $\alpha_{t}$ in section 2.3.1).

3. Prices Update: Once flows are updated, congestion control prices are updated as follows:

$$
\mu_{e}^{t}=\left[\mu_{e}^{t-1}+\rho\left(\lambda_{e}^{t}-\bar{\lambda}_{e}\right)\right]^{+}
$$

where $\rho>0$ and $\lambda_{e}^{t}=\lambda_{e}\left(\mathbf{r}^{t}\right)$. Externality prices are updated according to:

$$
\zeta_{e}^{t}=\lambda_{e}\left(\mathbf{r}^{t}\right) c_{e}^{\prime}\left(\lambda_{e}\left(\mathbf{r}^{t}\right)\right)
$$

4. Stopping Rule: If $\left\|\mathbf{d}^{t}-\mathbf{r}^{t-1}\right\|+\left\|\boldsymbol{\mu}^{t}-\boldsymbol{\mu}^{t-1}\right\|<\eta$ for some small $\eta>0$ the auction terminates and each user $m \in \mathcal{M}$ is assessed a payment of $P_{m}^{t}+\sum_{e \in \mathcal{E}} c_{e}^{t} \lambda_{e,-m}\left(\mathbf{r}^{t}\right)$ in return for the flow $r_{m}^{t}$ where

$$
\begin{aligned}
P_{m}^{t} & =P_{m}^{t-1}-\sum_{e \in \mathcal{E}}\left(c_{e}^{t-1}+\zeta_{e}^{t-1}+\mu_{e}^{t-1}\right)\left(d_{-m, e}^{t}-d_{-m, e}^{t-1}\right) \\
d_{-m, e}^{t} & =\sum_{\ell \in \mathcal{M} \backslash\{m\}} A_{\ell, e} d_{\ell}^{t} \\
\lambda_{e,-m}\left(\mathbf{r}^{t}\right) & =\sum_{\ell \in \mathcal{M} \backslash\{m\}} A_{\ell, e} r_{\ell}^{t}
\end{aligned}
$$

and $P_{m}^{0}=0$.

Let us now make some important remarks about this iterative auction:

- When users update their flow rate as in Eq.(2.5) using their truthful demand we say that they update truthfully. Clearly, since $U_{m}()$ is private information, only available to user $m \in \mathcal{M}$, any secondary user may deviate from this behavior and not update his flow rate truthfully. However in Theorem 2.3 we 


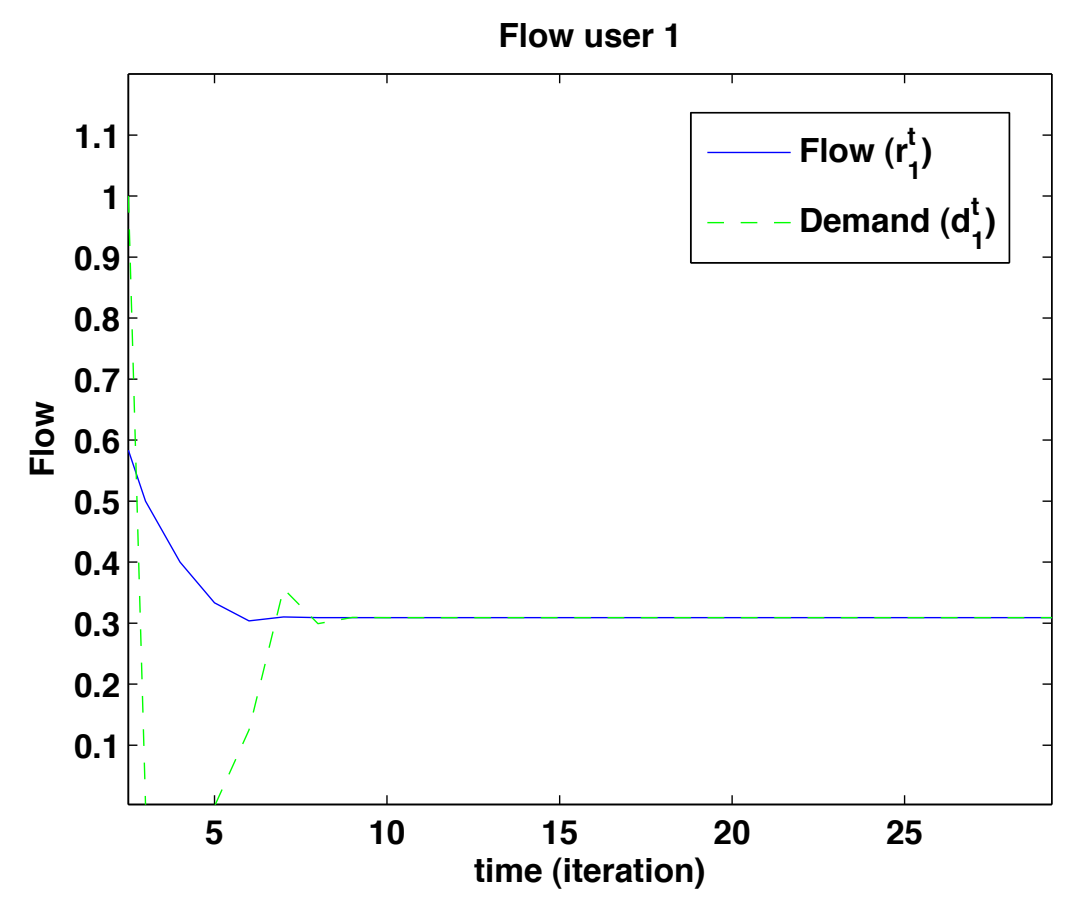

Figure 2.2: Flow demand of user 1 using ICA

state the incentives of each secondary user to do so, no matter the behavior of his competitors.

- In step 2 when updating his respective flow rate, each user indirectly reveals some demand which he claims is his truthful demand.

- When applying the algorithm described above to the problem used in the Literature Review, of a single link with unit capacity and two users (see Section 2.2) flow of user 1 is as shown in Fig. 2.2, user 2 has virtually the same behavior. Note that flows converge to $\left(r_{1}, r_{2}\right)=(0.31,0.31)$, the efficient flow configuration.

\subsubsection{About the Increasing Demand Inertia $\alpha_{t}$}

Note that $\frac{\rho}{\alpha_{t}} \rightarrow \infty$ which implies that the time-scale in which the flow assignment is updated is slower than that with which resource prices are updated. We posit the following additional conditions on the schedule $\left\{\alpha_{t}: t>0\right\}$ :

- $\alpha_{t} \in(0,1)$ 
- $\alpha_{t} \rightarrow 0^{+}$with $\alpha_{t+1}>\alpha_{t}$ for $t \geq 0$.

- $\sum_{t \geq 0} \alpha_{t} \rightarrow \infty$

- $\sum_{t \geq 0}\left(\alpha_{t}\right)^{\gamma}<\infty$ for any $\gamma>1$.

Since $\alpha_{t} \rightarrow 0$, flows are increasingly less responsive to demand. However since $\sum \alpha_{t} \rightarrow \infty$ any user can always make sure its flow approximates any desired value. This inertia allows users internalize the effects of the aggregate flow levels on their individual utility, avoiding the cycles observed when using iterative auctions that do not account for externalities.

\subsubsection{About the Externality Price $\zeta_{e}^{t}$}

The externality price will help each user internalize the effect of his individual flow on the marginal utility of his competitors. To see this, note that

$$
c_{e}^{t}+\zeta_{e}^{t}=c_{e}\left(\lambda_{e}\left(\mathbf{r}^{t}\right)\right)+\lambda_{e}\left(\mathbf{r}^{t}\right) c_{e}^{\prime}\left(\lambda_{e}\left(\mathbf{r}^{t}\right)\right)
$$

Furthermore, being truthful (see Eq.2.4) implies

$$
U_{m}^{\prime}\left(d_{m}^{t+1}\right)-\left(\mu_{e}^{t}+\zeta_{e}^{t}+c_{e}^{t}\right) \leq 0
$$

with equality if $d_{m}^{t+1}>0$. Hence, if $\mathbf{d}^{t} \rightarrow \mathbf{r}^{*}, \mathbf{r}^{t} \rightarrow \mathbf{r}^{*}$ and $\boldsymbol{\mu} \rightarrow \boldsymbol{\mu}^{*}$ for some $\mathbf{r}^{*} \in \mathbb{R}_{+}^{M}$ and $\boldsymbol{\mu}^{*} \in \mathbb{R}_{+}^{E}$, the limit configuration, $\mathbf{r}^{*}$, holds efficiency conditions in Eq.(2.2.a)

\subsubsection{About Payment Rule}

In this subsection we give some intuition about the purpose of the payment rule of ICA. In step 4 of the pseudocode above, we stated that if the auction closes at time $T$, user $m \in \mathcal{M}$ is assessed a payment of $P_{m}^{T}+\sum_{e \in \mathcal{E}} c_{e}^{T} \lambda_{e,-m}\left(\mathbf{r}^{T}\right)$. Let us start by considering the term $P_{m}^{T}$ which is described in detail in Eq.(2.8) and which we shall refer to as the cumulative payment of user $m \in \mathcal{M}$. 
Note that $P_{m}^{T}$ can be interpreted as a cumulative payment where at any iteration $t \leq T$, user $m$ pays the change in his competitors demand for capacity on each link $e \in \mathcal{E}$ (i.e. $d_{-m, e}^{t-1}-d_{-m, e}^{t}$ ) at price $\left[\mu_{e}^{t-1}+\zeta_{e}^{t-1}+c_{e}^{t-1}\right]$. As we shall formally prove later, this payment approximates the change in aggregate intrinsic utility of $m^{\prime} s$ competitors if their flow rates change from $\left\{d_{\ell}^{t}\right\}_{\ell \neq m}$ to $\left\{d_{\ell}^{t-1}\right\}_{\ell \neq m}$. That is, payment at time $t \leq T$ approximates $\left(\sum_{\ell \neq m} U_{\ell}\left(d_{\ell}^{t-1}\right)-\sum_{\ell \neq m} U_{\ell}\left(d_{\ell}^{t}\right)\right)$, hence the aggregate payment from $t=1$ to $t=T$ approximates $\left(\sum_{\ell \neq m} U_{\ell}\left(d_{\ell}^{0}\right)-\sum_{\ell \neq m} U_{\ell}\left(d_{\ell}^{T}\right)\right)$. We already know that $d_{\ell}^{0}=0$, therefore if at iteration $T$ the demand profile $\mathbf{d}^{T}$ has converged to $\mathbf{r}^{T} \in \mathbb{R}_{+}^{M}$, the total payment of user $m \in \mathcal{M}$ is

$$
P_{m}^{T}+\sum_{e \in \mathcal{E}} c_{e}^{T} \lambda_{e,-m}\left(\mathbf{r}^{T}\right) \approx \sum_{\ell \neq m} U_{\ell}(0)-\sum_{\ell \neq m}\left[U_{\ell}\left(r_{\ell}^{T}\right)-r_{\ell}^{T} \sum_{e \in \mathcal{E}} A_{m, e} c_{e}\left(\lambda_{e}\left(\mathbf{r}^{T}\right)\right)\right]
$$

Note that this payment will resemble the negative of the utility of $m$ 's competitors (i.e. $\left.-\sum_{\ell \neq m}\left[U_{\ell}\left(r_{\ell}^{T}\right)-r_{\ell}^{T} \sum_{e \in \mathcal{E}} A_{\ell, e} c_{e}\left(\lambda_{e}\left(\mathbf{r}^{T}\right)\right)\right]\right)$ plus a constant $\sum_{\ell \neq m} U_{\ell}(0)$.

This payment will align $k$ 's profit with the manager's objective of implementing the socially efficient flow profile as defined in Eq.(2.2). That is, each user will maximize his profit when the efficient flow configuration is implemented, which in turn is guaranteed by updating flow profiles truthfully. These results are formally stated and proved in sections 2.5 and 2.6.

\subsection{Assumptions}

The standing assumptions for the analysis of the Inertial Capacity Auction are as follows.

Assumption 1: For each user $m \in \mathcal{M}, U_{m}()$ is increasing, strongly concave and twice continuously differentiable.

Assumption 2: For each link $e \in \mathcal{E}, c_{e}$ is convex, strictly increasing and twice continuously differentiable 
Assumption 3: There exists a "choking" price $\bar{\mu}>0$ such that for any $p \geq \bar{\mu}$, $d_{m}=0$ for all $m \in \mathcal{M}$ where

$$
d_{m}=\arg \max _{d \in \mathbb{R}_{+}}\left[U_{m}(d)-p d\right]
$$

Assumption 4: Each user $m \in \mathcal{M}$ has a maximum demand, $\bar{r}_{m}>0$ such that $\bar{r}_{m}>r_{m}^{*}$, where $\mathbf{r}^{*}=\left[r_{1}^{*}, \ldots, r_{M}^{*}\right]$ is the solution of the optimization problem in (Eq. 2.2). This in turn implies the existence of a maximum demand for each link $e \in \mathcal{E}$, say $\bar{d}_{e}>0$.

\subsection{Price and Flow profile Convergence}

\subsubsection{Convergence of Control Price Profile $\boldsymbol{\mu}^{t}$}

In this section, we analyze the stability of the proposed mechanism assuming that all users update their demand truthfully, that is $r_{m}^{t}=r_{m}^{t-1}+\alpha_{t}\left(d_{m}^{t}-r_{m}^{t-1}\right)$ where $d_{m}^{t}$ is as in Eq.(2.4).

The following Lemma is a preliminary result which will be useful to prove convergence of prices $\mu_{e}^{t}$ for all $e \in \mathcal{E}$.

Lemma 2.1 (Boundedness of $\lambda_{e}^{t}$ ). $\limsup _{t \rightarrow \infty} \lambda_{e}^{t} \leq \overline{\lambda_{e}}$, for each $e \in \mathcal{E}$.

Proof: For the formal proof we refer the reader to the appendix. In what follows, we provide a sketch of the proof. Suppose $\lambda_{e}^{t} \rightarrow \infty$ or $\lambda_{e}^{t} \rightarrow \lambda_{e}^{*}>\bar{\lambda}_{e}$, this implies $\mu_{e}^{t} \rightarrow \infty$, which in turn implies $d_{m}^{t} \rightarrow 0$ for all $m \in \mathcal{M}$ with $A_{m, e}>0$,

therefore $\lambda_{e}^{t} \rightarrow 0$, a contradiction. Now assume $\lambda_{e}^{t}$ oscillates in the limit (i.e. $\left.\lim \sup _{t \rightarrow \infty} \lambda_{e}^{t}>\liminf \operatorname{in}_{t \rightarrow \infty} \lambda_{e}^{t}\right)$ with $\lim \sup _{t \rightarrow \infty} \lambda_{e}^{t}>\overline{\lambda_{e}}$. This implies the existence of an interval $\left[X_{1}, X_{2}\right]$ with $\overline{\lambda_{e}}<X_{1}<X_{2}$ which is up-crossed infinitely often. Because of increasing inertia in the flow assignment process, prices are to increase without bound, i.e. $\mu_{e}^{t} \rightarrow+\infty$, as $\lambda_{e}^{t}$ approaches $X_{2}$ (from $X_{1}$ ), which is contradiction to Assumption 3 ("choking" prices). 
Theorem 2.1: (Price Convergence) $\mu_{e}^{t} \rightarrow \mu_{e}^{*} \geq 0$, for each $e \in \mathcal{E}$.

Proof: Suppose the sequence $\left\{\mu_{e}^{t}: t>0\right\}$ does not converge. Therefore, either $\mu_{e}^{t} \rightarrow \infty$ or it oscillates with $\lim \inf \mu_{e}^{t}<\lim \sup \mu_{e}^{t}$. The former is easily discarded since large prices will result in null demand $\left(d_{m}^{t}=0\right.$ for all $m \in \mathcal{M}$ with $A_{m, e}>0$ ) leading to $\lambda_{e}^{t} \rightarrow 0$ which in turn implies $\mu_{e}^{t} \rightarrow 0$ which is a contradiction. Now assume that $\liminf \mu_{e}^{t}<\lim \sup \mu_{e}^{t}$, this price oscillation is only possible if the aggregate flow on link $e \in \mathcal{E}, \lambda_{e}^{t}$, oscillates around $\bar{\lambda}_{e}$ with $\liminf \lambda_{e}^{t}<\bar{\lambda}_{e}<\limsup \lambda_{e}^{t}$, which contradicts Lemma 2.1.

The following Corollaries are preliminary results to the proof of convergence of the flow profile to the efficient flow configuration (Theorem 2.2).

Corollary 2.1: (Complementary Slackness). $\mu_{e}^{t}\left(\bar{\lambda}_{e}-\lambda_{e}^{t}\right) \rightarrow 0$ for each $e \in \mathcal{E}$

Proof: If $\mu_{e}^{t}$ converges to zero (i.e. $\mu_{e}^{t} \rightarrow \mu_{e}^{*}=0$ ), Lemma 1.1 directly implies $\mu_{e}^{t}\left(\overline{\lambda_{e}}-\lambda_{e}^{t}\right) \rightarrow 0$. If it converges to a positive value (i.e. $\mu_{e}^{t} \rightarrow \mu_{e}^{*}>0$ ) then the price adjustment rule in Eq.( 2.6) implies $\lambda_{e}^{t} \rightarrow \bar{\lambda}_{e}$, hence the result.

Note that by construction $\mu_{e}^{t} \geq 0$ for all $e \in \mathcal{E}$ and $r_{m} \geq 0$ for all $m \in \mathcal{M}$. Furthermore Corollary 2.1 guarantees that in the limit, condition in Eq. (2.2.b) is held. The following 2 corollaries are preliminary results to the proof of Theorem 2.2 in which we show that condition in Eq.(2.2.a) is also held.Hence the limit configuration is the efficient one.

Corollary 2.2: $\sum_{t>0} \alpha_{t}\left|\mu_{e}^{t}-\mu_{e}^{*}\right| \quad$ for all $e \in \mathcal{E}$.

Proof: See appendix.

Corollary 2.3: $\quad 0 \leq \sum_{t>0}\left(\mu_{e}^{t+1}-\mu_{e}^{t}\right)\left(\lambda_{e}^{t+1}-\bar{\lambda}_{e}\right)<\infty, \quad e \in \mathcal{E}$.

Proof: See appendix.

In order to give some clues on the importance of these corollaries let us describe briefly some of the findings we will cover in detail in the following subsection. In subsection 2.5.2 we study the dynamics of the flow profile $\mathbf{r}^{t}$ by considering 
a function that approximates the social surplus for large values of $t$. We study the change in that approximate social surplus as the effect of updating flows and control prices. The effect of prices update at iteration $t$ will be of the form $\left(\mu_{e}^{t+1}-\mu_{e}^{t}\right)\left(\lambda_{e}^{t+1}-\bar{\lambda}_{e}\right)$. Corollary 3 builds upon Corollary 2 and states that the aggregate effect of this updates throughout the auction is bounded.

\subsubsection{Convergence of Flow profile $\mathrm{r}^{t}$ and Efficiency of the Limit Configuration}

Having established that prices converge, we analyze the convergence of individual flows to the efficient configuration. Consider the function $\Psi: \mathbb{R}_{+}^{M} \times \mathbb{R}_{+}^{E} \rightarrow \mathbb{R}$ defined as follows:

$$
\Psi(\mathbf{r}, \boldsymbol{\mu})=\sum_{m \in \mathcal{M}} U_{m}\left(r_{m}\right)-\sum_{e \in \mathcal{E}} \lambda_{e}(\mathbf{r}) c_{e}\left(\lambda_{e}(\mathbf{r})\right)+\sum_{e \in \mathcal{E}}\left(\overline{\lambda_{e}}-\lambda_{e}(\mathbf{r})\right) \mu_{e}
$$

In light of Corollary 1 (Complementary slackness), $\Psi\left(\mathbf{r}^{t}, \boldsymbol{\mu}^{t}\right)$ is a good approximation of social surplus for large values of $t>0$.

The following proposition states that our algorithm is steepest ascend in the approximate social surplus, in the sense that the direction of relative demand $\left(\mathbf{d}^{t+1}-\mathbf{r}^{t}\right)$ is close to the direction of the gradient $\nabla_{\mathbf{r}} \Psi\left(\mathbf{r}^{t}, \boldsymbol{\mu}^{t}\right)$.

Proposition 2.1 There exist some $M>0$ such that $\left(\mathbf{d}^{t+1}-\mathbf{r}^{t}\right) . \nabla_{\mathbf{r}} \Psi\left(\mathbf{r}^{t}, \boldsymbol{\mu}^{t}\right) \geq$ $M\left\|\mathbf{d}^{t+1}-\mathbf{r}^{t}\right\|^{2} \geq 0$

Proof: It will suffice to prove that for each $m \in \mathcal{M}$ there exists some $K_{m}>0$ such that

$$
\left(d_{m}^{t+1}-r_{m}^{t}\right) \frac{\partial \Psi}{\partial r_{m}}\left(\mathbf{r}^{t}, \boldsymbol{\mu}^{t}\right) \geq K_{m}\left(d_{m}^{t+1}-r_{m}^{t}\right)^{2}
$$

Note that $\frac{\partial \Psi}{\partial r_{m}}\left(\mathbf{r}^{t}, \boldsymbol{\mu}^{t}\right)=U_{m}^{\prime}\left(r_{m}^{t}\right)-p_{m}^{t+1}$ and according to demand rule in Eq.(2.4) $U_{m}^{\prime}\left(d_{m}^{t+1}\right) \leq p_{m}^{t+1}$ with equality if $d_{m}^{t+1}>0$. We now consider two cases:

- Consider the case $d_{m}^{t+1}=0$ : In this case we have that $p_{m}^{t+1} \geq U_{m}^{\prime}(0) \geq U_{m}^{\prime}\left(r_{m}^{t}\right)$ hence $p_{m}^{t+1}-U_{m}^{\prime}\left(r_{m}^{t}\right) \geq U_{m}^{\prime}(0)-U_{m}^{\prime}\left(r_{m}^{t}\right)$, therefore 


$$
\begin{aligned}
\left(d_{m}^{t+1}-r_{m}^{t}\right) \frac{\partial \Psi}{\partial r_{m}}\left(\mathbf{r}^{t}, \boldsymbol{\mu}^{t}\right) & =\left(-r_{m}^{t}\right)\left(U_{m}^{\prime}\left(r_{m}^{t}\right)-p_{m}^{t+1}\right) \\
& \geq r_{m}^{t}\left(U_{m}^{\prime}(0)-U_{m}^{\prime}\left(r_{m}^{t}\right)\right) \\
& \geq K_{m}\left(r_{m}^{t}\right)^{2}
\end{aligned}
$$

where the last inequality comes from Assumption 1.

- Consider now the case $d_{m}^{t+1}>0$ : In this case we have $p_{m}^{t+1}=U_{m}^{\prime}\left(d_{m}^{t+1}\right)$ and

$$
\begin{aligned}
\left(d_{m}^{t+1}-r_{m}^{t}\right) \frac{\partial \Psi}{\partial r_{m}}\left(\mathbf{r}^{t}, \boldsymbol{\mu}^{t}\right) & =\left(d_{m}^{t+1}-r_{m}^{t}\right)\left(U_{m}^{\prime}\left(r_{m}^{t}\right)-U_{m}^{\prime}\left(d_{m}^{t}\right)\right) \\
& \geq K_{m}\left(d_{m}^{t+1}-r_{m}^{t}\right)^{2}
\end{aligned}
$$

where again, the last inequality comes from Assumption 1.

In what follows we use the result of Proposition 2.1 to prove the convergence of the social surplus approximation $\Psi\left(\mathbf{r}^{t}, \boldsymbol{\mu}^{t}\right)$ to some level $\widetilde{\Psi} \in \mathbb{R}$. Before that let us make some important observations.

Note that Assumption 4 (bounded demand) altogether with flow adjustment rule in Eq.(2.5) implies the existence of a compact set $\mathbb{D} \subset \mathbb{R}^{M}$ such that $\mathbf{r}^{t}, \mathbf{d}^{t} \in \mathbb{D}$ for all $t \geq 0$. Assumptions 1 and 2 imply $\nabla_{\mathbf{r}} \Psi$ is Lipschitz continuous in $\mathbb{D}$, therefore there exist some $L>0$ such that:

$$
\left|\nabla_{\mathbf{r}} \Psi(\mathbf{r}, \boldsymbol{\mu})-\nabla_{\mathbf{r}} \Psi(\widetilde{\mathbf{r}}, \boldsymbol{\mu})\right| \leq L\|\mathbf{r}-\widetilde{\mathbf{r}}\|
$$

for all $\mathbf{r}, \widetilde{\mathbf{r}} \in \mathbb{D}, \boldsymbol{\mu} \in \mathbb{R}_{+}^{E}$. This in turn implies:

$$
\left|\Psi(\widetilde{\mathbf{r}}, \boldsymbol{\mu})-\Psi(\mathbf{r}, \boldsymbol{\mu})-(\widetilde{\mathbf{r}}-\mathbf{r}) \cdot \nabla_{\mathbf{r}} \Psi(\mathbf{r}, \boldsymbol{\mu})\right| \leq \frac{L}{2}\|\widetilde{\mathbf{r}}-\mathbf{r}\|^{2}
$$

for all $\mathbf{r}, \widetilde{\mathbf{r}} \in \mathbb{D}, \boldsymbol{\mu} \in \mathbb{R}_{+}^{E}$. Proof of Eq.(2.10) can be found in many convex optimization textbooks, hence it is omitted in this work. The following proposition uses this property to prove convergence of the approximate social surplus $\Psi\left(\mathbf{r}^{t}, \boldsymbol{\mu}^{t}\right)$. 
Proposition 2.2: (Approximate Surplus Convergence). $\Psi\left(\mathbf{r}^{t}, \boldsymbol{\mu}^{t}\right) \rightarrow$ $\widetilde{\Psi} \in \mathbb{R}$

Proof: Let $\boldsymbol{\lambda}^{t}=\left[\lambda_{1}^{t}, \ldots, \lambda_{E}^{t}\right]$, from Eq. (2.10) and Proposition 2.1, it follows that:

$$
\begin{aligned}
\Psi\left(\mathbf{r}^{t+1}, \boldsymbol{\mu}^{t+1}\right)-\Psi\left(\mathbf{r}^{t}, \boldsymbol{\mu}^{t}\right)= & \Psi\left(\mathbf{r}^{t+1}, \boldsymbol{\mu}^{t+1}\right)-\Psi\left(\mathbf{r}^{t+1}, \boldsymbol{\mu}^{t}\right)+\Psi\left(\mathbf{r}^{t+1}, \boldsymbol{\mu}^{t}\right)-\Psi\left(\mathbf{r}^{t}, \boldsymbol{\mu}^{t}\right) \\
\geq & \left(\boldsymbol{\mu}^{t+1}-\boldsymbol{\mu}^{t}\right)\left(\overline{\boldsymbol{\lambda}}-\lambda^{t+1}\right)+\left(\mathbf{r}^{t+1}-\mathbf{r}^{t}\right) \cdot \nabla_{\mathbf{r}} \Psi\left(\mathbf{r}^{t}, \boldsymbol{\mu}^{t}\right) \\
& -\frac{L}{2}\left\|\mathbf{r}^{t+1}-\mathbf{r}^{t}\right\|^{2} \\
\geq & \left(\boldsymbol{\mu}^{t+1}-\boldsymbol{\mu}^{t}\right)\left(\overline{\boldsymbol{\lambda}}-\lambda^{t+1}\right)+\alpha_{t}\left(\mathbf{d}^{t+1}-\mathbf{r}^{t}\right) \cdot \nabla_{\mathbf{r}} \Psi\left(\mathbf{r}^{t}, \boldsymbol{\mu}^{t}\right) \\
& -\frac{L}{2} \alpha_{t}^{2}\left\|\mathbf{d}^{t+1}-\mathbf{r}^{t}\right\|^{2} \\
\geq & -\left(\boldsymbol{\mu}^{t+1}-\boldsymbol{\mu}^{t}\right)\left(\boldsymbol{\lambda}^{t+1}-\overline{\boldsymbol{\lambda}}\right)-\frac{L}{2} \alpha_{t}^{2}\|\overline{\mathbf{r}}\|^{2}
\end{aligned}
$$

where $\overline{\mathbf{r}}=\left[\bar{r}_{1}, \ldots, \bar{r}_{M}\right]$ is the vector of maximum demand (Assumption 4). From Corollary 2.3 it follows that $\Psi\left(\mathbf{r}^{t}, \boldsymbol{\mu}^{t}\right)$ does not go to $-\infty$ neither it oscillates. Thus boundedness (above) of $\Psi\left(\mathbf{r}^{t}, \boldsymbol{\mu}^{t}\right)$ implies convergence.

We are now ready to prove main result of this section, efficiency of the limit flow configuration.

Theorem 2.2 $\mathbf{r}^{t} \rightarrow \mathbf{r}^{*} \in \mathbb{R}_{+}^{M}$ where $\boldsymbol{r}^{*}$ is the unique solution of problem in Eq. (2.2).

Proof: In the proof of Theorem 2.2 we already showed that

$$
\begin{aligned}
\Psi\left(\mathbf{r}^{t+1}, \boldsymbol{\mu}^{t+1}\right)-\Psi\left(\mathbf{r}^{t}, \boldsymbol{\mu}^{t}\right) \geq & \left(\boldsymbol{\mu}^{t+1}-\boldsymbol{\mu}^{t}\right)\left(\overline{\boldsymbol{\lambda}}-\boldsymbol{\lambda}^{t+1}\right)+\alpha_{t}\left(\mathbf{d}^{t+1}-\mathbf{r}^{t}\right) \cdot \nabla_{\mathbf{r}} \Psi\left(\mathbf{r}^{t}, \boldsymbol{\mu}^{t}\right) \\
& -\frac{L}{2} \alpha_{t}^{2}\left\|\mathbf{d}^{t+1}-\mathbf{r}^{t}\right\|^{2}
\end{aligned}
$$

From Proposition 2.2 and Corollary 2.3 it follows that

$$
\begin{aligned}
\sum_{t>0} \alpha_{t}\left(\mathbf{d}^{t+1}-\mathbf{r}^{t}\right) \cdot \nabla_{\mathbf{r}} \Psi\left(\mathbf{r}^{t}, \boldsymbol{\mu}^{t}\right) \leq & \widetilde{\Psi}-\Psi\left(\mathbf{r}^{0}, \boldsymbol{\mu}^{0}\right)-\sum_{t>0}\left(\boldsymbol{\mu}^{t+1}-\boldsymbol{\mu}^{t}\right)\left(\overline{\boldsymbol{\lambda}}-\lambda^{t+1}\right) \\
& +\frac{L}{2} \alpha_{t}^{2}\|\overline{\mathbf{r}}\|^{2} \\
< & \infty
\end{aligned}
$$


Hence, $\left(\mathbf{d}^{t+1}-\mathbf{r}^{t}\right) \cdot \nabla_{\mathbf{r}} \Psi\left(\mathbf{r}^{t}, \boldsymbol{\mu}^{t}\right) \rightarrow 0$. Which in light of Theorem 1 (price convergence) and truthful demand becomes $\left(\mathbf{d}^{t+1}-\mathbf{r}^{t}\right) \cdot \nabla_{\mathbf{r}} \Psi\left(\mathbf{r}^{t}, \boldsymbol{\mu}^{*}\right) \rightarrow 0$. Finally Proposition 2.1 gives us $\left\|\mathbf{d}^{t+1}-\mathbf{r}^{t}\right\| \rightarrow 0$. This, altogether with truthful demand (see Eq.2.4) implies that for $t \rightarrow \infty$

$$
U_{m}^{\prime}\left(r_{m}^{t}\right)-\left(\mu_{e}^{*}+c_{e}\left(\lambda_{e}\left(\mathbf{r}^{t}\right)\right)+\lambda_{e}\left(\mathbf{r}^{t}\right) c_{e}^{\prime}\left(\lambda_{e}\left(\mathbf{r}^{t}\right)\right)\right) \leq 0
$$

for all $m \in \mathcal{M}$ with equality if $r_{m}^{t}>0$, which resembles condition in Eq.(2.2.a). Since condition in Eq.(2.2.b) is guaranteed in Corollary 2.1, the limit flow configuration, say $\mathbf{r}^{*}$ is the efficient one (i.e. the solution to problem in Eq.2.2).

\subsection{Incentive Compatibility}

In this section we revisit the assumption that all users update their flow rate truthfully (i.e. according to Eq.2.4 and 2.5) based upon which the convergence and efficiency of the resulting flows has been established.

Note that the network manager (auctioneer) can impose an activity rule requiring that flow demands of each user $m \in \mathcal{M}$ must be consistent with some function $\widetilde{U}_{m}() \in \mathbb{U}$, where $\mathbb{U}$ is the set of strongly concave functions from $\mathbb{R}_{+}$to $\mathbb{R}$. In other words, for each $m \in \mathcal{M}$ there must exist some $\widetilde{U}_{m}() \in \mathbb{U}$ such that

$$
d_{m}^{t}=\underset{d \geq 0}{\operatorname{argmax}}\left[\widetilde{U}_{m}(d)-d p_{m}^{t}\right]
$$

for all $t>0$. Note that the auctioneer can enforce this rule by simply checking that larger prices correspond to lower demands (i.e. for any $t, t^{\prime}>0$ and $m \in \mathcal{M}$ it must hold that $p_{m}^{t}<p_{m}^{t^{\prime}}$ implies $d_{m}^{t} \geq d_{m}^{t^{\prime}}$ with equality only if $d_{m}^{t}=d_{m}^{t^{\prime}}=0$ ). Since $\mu_{e}^{0}=\bar{\mu}$, an additional sensible activity rule is to require $d_{m}^{1}=0$ for all $m \in \mathcal{M}$

Any user violating any of these activity rules will be penalized with a large 
payment and removed from the auction. This penalization will guarantee that all users will update in a way that is consistent with some utility function from the space $\mathbb{U}$. In what follows we shall consider a strategy by user $m \in \mathcal{M}$ in which he behaves as if his utility function were $\widetilde{U}_{m} \in \mathbb{U}$. Let us start by characterizing payments when such strategies are followed.

Recall that if the auction ends at iteration $t>0$ payment of user $m \in \mathcal{M}$ is $P_{m}^{t}+\sum_{e \in \mathcal{E}} c_{e}^{t} \lambda_{e,-m}\left(\mathbf{r}^{t}\right)$. As noted in Section 2.3.3 the term $P_{m}^{t}$ has a cumulative nature, hence we call it cumulative payment. The following Lemma describes the resulting cumulative payments to be assessed on users when they adjust their flow rates according to intrinsic utility functions $\left\{\widetilde{U}_{m} \in \mathbb{U}: m \in \mathcal{M}\right\}$. Let $\widetilde{P}_{m}=\lim _{t \rightarrow \infty} P_{m}^{t}$ denote this payment for user $m \in \mathcal{M}$. The characterization of these payments will be used later to show that for all users, it is a dominant strategy to adjust flow rates truthfully. That is, when $\widetilde{U}_{m}()$ is set equal to the actual utility function $U_{m}()$.

Lemma 2.2: If users employ utility functions $\left\{\widetilde{U}_{m} \in \mathbb{U}: m \in \mathcal{M}\right\}$ to calculate demands as in Eq. (2.11), then flows and prices converge (i.e. $\mathbf{r}^{t} \rightarrow \widetilde{\mathbf{r}} \in R_{+}^{M}$, $\mu^{t} \rightarrow \widetilde{\mu} \in R_{+}^{E}$ ) and the cumulative payment of user $m \in \mathcal{M}$ converges to

$$
\widetilde{P}_{m}=\sum_{\ell \in \mathcal{M} \backslash\{m\}} \widetilde{U}_{\ell}(0)-\sum_{\ell \in \mathcal{M} \backslash\{m\}} \widetilde{U}_{\ell}\left(\widetilde{r}_{\ell}\right)+\widetilde{\delta}_{m}(\rho)
$$

where $\widetilde{\delta}_{m}(\rho): \mathbb{R}_{+} \mapsto \mathbb{R}$ is a mapping such that $\left|\widetilde{\delta}_{m}(\rho)\right| \rightarrow 0^{+}$as $\rho \rightarrow 0^{+}$.

Proof: The formal proof is given in the appendix. Here we provide a sketch.

Prices and flows convergence are immediate by Theorems 2.1 and 2.2 when bidders calculate demands as in Eq.(2.11) according to intrinsic utility functions $\left\{\widetilde{U}_{m}: m \in \mathcal{M}\right\}$. The cumulative payment characterization however, is not that trivial.

At iteration $t>0$, bidder $m \in \mathcal{M}$ pays the change in his competitors demand for capacity on link $e \in \mathcal{E}$ (i.e. $\left.\left(d_{-m, e}^{t-1}-d_{-m, e}^{t}\right)\right)$ at price $p_{e}^{t}$. Following a similar analysis as in Section 2.3.3 it follows that in the limit, user $m \in \mathcal{M}$ is assessed a 
total payment that approximates the aggregate change in the intrinsic utility of his competitors as measured by $\left\{\widetilde{U}_{\ell}: \ell \in \mathcal{M} \backslash\{m\}\right\}$. That is, the aggregate payment of user $m \in \mathcal{M}$ approximates $\left(\sum_{\ell \neq m} \widetilde{U}_{\ell}\left(d_{\ell}^{1}\right)-\sum_{\ell \neq m} \widetilde{U}_{\ell}\left(d_{\ell}^{T}\right)\right)$. The term $\widetilde{\delta}_{m}(\rho)$ is an approximation error that emerges as a result of having discrete changes in prices and demands. Since an infinitesimal $\rho>0$ yields infinitesimal changes in prices and demands, the approximation error is determined by the magnitude of $\rho>0$. Note that we use $\widetilde{\mathbf{r}}, \widetilde{\delta}(\rho)$ and $\widetilde{P}_{m}$ to highlight the fact that the resultant flows, approximation error and payments will depend on the functions $\left\{\widetilde{U}_{m}: m \in \mathcal{M}\right\}$ used.

At this point we are interested in the overall outcome of the auction, mainly we want to characterize the final joint flow profile and payments. We are specially interested in the effects of removing users from the auction.

Let $\widetilde{\mathcal{M}}$ be the set of users that are active at the end of the auction (i.e. those that followed the activity rules until the final iteration) and let $\widetilde{U}_{\ell}$ be the strongly concave function that characterizes the demand history of user $\ell \in \widetilde{\mathcal{M}}$. By the same arguments used in Theorems 2.1 and 2.2 the final allocation, say $\widetilde{\mathbf{r}}=\left[\widetilde{r}_{1}, \ldots, \widetilde{r}_{M}\right]$, does not depend on the initial conditions, it will be the efficient allocation for those users that are active at the end of the auction (note that this efficiency is relative to the functions $\left.\left\{\widetilde{U}_{m}(): m \in \widetilde{\mathcal{M}}\right\}\right)$. Formally, the limit flow profile $\widetilde{\mathbf{r}}$, is the solution to the following problem :

$$
\begin{gathered}
\max _{\mathbf{r} \in \mathbb{R}_{+}^{M}}\left[\sum_{m \in \widetilde{\mathcal{M}}} \widetilde{U}_{m}\left(r_{m}\right)-\sum_{e \in \mathcal{E}} \lambda_{e}(\mathbf{r}) c_{e}(\mathbf{r})\right] \\
\text { s.t. } \quad \lambda_{e}(\mathbf{r}) \leq \bar{\lambda}_{e} \quad e \in \mathcal{E}
\end{gathered}
$$

where clearly $\widetilde{r}_{\ell}=0$ for all $\ell \notin \widetilde{\mathcal{M}}$. From Lemma 2.2 it can be noticed that the payment of user $m \in \mathcal{M}$ is designed to align $m$ 's incentives with those of the benevolent network manager (maximize the aggregate utility of the users). Since 
this benevolent manager does not care about utility of users that have been removed from the auction, payment of active users should not consider such competitors either, hence a simple modification on the payment rule is required. Payment of user $m \in \widetilde{\mathcal{M}}$ if the auction terminates at iteration $t>0$, should be redefined as $P_{m}^{t}+\sum_{e \in \mathcal{E}} c_{e}^{t} \lambda_{e,-m}\left(\mathbf{r}^{t}\right)$ where

$$
\begin{aligned}
P_{m}^{t} & =P_{m}^{t-1}-\sum_{e \in \mathcal{E}} p_{e}^{t}\left(d_{-m, e}^{t}-d_{-m, e}^{t-1}\right) \\
d_{-m, e}^{t} & =\sum_{\ell \in \widehat{\mathcal{M}} \backslash\{m\}} A_{\ell, e} d_{\ell}^{t} \\
\lambda_{e,-m}\left(\mathbf{r}^{t}\right) & =\sum_{\ell \in \widetilde{\mathcal{M}} \backslash\{m\}} A_{\ell, e} r_{\ell}^{t}
\end{aligned}
$$

for $t>0$ and $P_{m}^{0}=0$. Following a similar analysis as in Lemma 2.2 it is easy to see that cumulative payment of user $m \in \widetilde{\mathcal{M}}$ will be:

$$
\widetilde{P}_{m}=\sum_{\ell \in \widetilde{\mathcal{M}} \backslash\{m\}} \widetilde{U}_{\ell}(0)-\sum_{\ell \in \widetilde{\mathcal{M}} \backslash\{m\}} \widetilde{U}_{\ell}\left(\widetilde{r}_{\ell}\right)+\widetilde{\delta}_{m}(\rho)
$$

Note that properties of the overall results (allocation and payments) are not altered by the participation of users that were removed from the auction. Hence efficiency of the limit flow profile and the characterization of payments in Lemma 2.2 hold true as if only users $\widetilde{\mathcal{M}} \subseteq \mathcal{M}$ were active since the beginning of the auction.

Theorem 2.3: In the Inertial Capacity Auction being truthful is an $\epsilon$-Dominant strategy in the sense that, the maximum benefit any user $m \in \mathcal{M}$ can achieve by not being truthful, is $\epsilon(\rho)$ where $\epsilon(\rho) \rightarrow 0$ as $\rho \rightarrow 0^{+}$

Proof: Again let $\widetilde{\mathcal{M}}$ be the set of users that are active at the end of the auction and let $\widetilde{U}_{m}$ be the strongly concave function that characterizes the demand history of user $m \in \widetilde{\mathcal{M}}$. The resultant allocation $\widetilde{\mathbf{r}}$ has already been described in Eq.(2.12). By the characterization of $\widetilde{P}_{m}$ in Eq. (2.13) the net profit of user $m$ (which we 
denote by $\left.\widetilde{V}_{m}\right)$ is:

$$
\begin{aligned}
\widetilde{V}_{m} & =U_{m}\left(\widetilde{r}_{m}\right)-\widetilde{r}_{m} \sum_{e \in \mathcal{E}} A_{m, e} c_{e}(\widetilde{\mathbf{r}})-\lim _{t \rightarrow \infty}\left[P_{m}^{t}+\sum_{e \in \mathcal{E}} c_{e}^{t} \lambda_{e,-m}\left(\mathbf{r}^{t}\right)\right] \\
& =U_{m}\left(\widetilde{r}_{m}\right)-\sum_{e \in \mathcal{E}} \lambda_{e}(\widetilde{\mathbf{r}}) c_{e}\left(\lambda_{e}(\widetilde{\mathbf{r}})\right)-\widetilde{P}_{m} \\
& =U_{m}\left(\widetilde{r}_{m}\right)+\sum_{\ell \in \widetilde{\mathcal{M}} \backslash\{m\}} \widetilde{U}_{\ell}\left(\widetilde{r}_{\ell}\right)-\sum_{e \in \mathcal{E}} \lambda_{e}(\widetilde{\mathbf{r}}) c_{e}\left(\lambda_{e}(\widetilde{\mathbf{r}})\right)-\sum_{\ell \in \widetilde{\mathcal{M}} \backslash\{m\}} \widetilde{U}_{\ell}(0)-\widetilde{\delta}_{m}(\rho)
\end{aligned}
$$

Now consider the case in which user $m \in \widetilde{\mathcal{M}}$ reports his demand truthfully (setting $\widetilde{U}_{m}=U_{m}$ ) while all other users $\ell \neq m$ do so according to Eq. (2.11). Abusing notation, let us denote by $\widehat{\mathbf{r}} \in \mathbb{R}_{+}^{M}$ the limiting flow profile and by $\widehat{P}_{m}$ the cumulative payment of user $m$. Here again by arguments similar to those used in Theorems 2.1 and 2.2 it can be shown that $\widehat{\mathbf{r}}$ is the solution to

$$
\begin{gathered}
\max _{\mathbf{r} \in \mathbb{R}_{+}^{M}}\left[U_{m}\left(r_{m}\right)+\sum_{\ell \in \widetilde{\mathcal{M}} \backslash\{m\}} \widetilde{U}_{\ell}\left(r_{\ell}\right)-\sum_{e \in \mathcal{E}} \lambda_{e}(\mathbf{r}) c_{e}(\mathbf{r})\right] \\
\text { s.t. } \quad \lambda_{e}(\mathbf{r}) \leq \bar{\lambda}_{e} \quad e \in \mathcal{E}
\end{gathered}
$$

Similar as in Eq.(2.13), the cumulative payment of user $m$ is:

$$
\widehat{P}_{m}=\sum_{\ell \in \widetilde{\mathcal{M}} \backslash\{m\}} \widetilde{U}_{\ell}(0)-\sum_{\ell \in \widetilde{\mathcal{M}} \backslash\{m\}} \widetilde{U}_{\ell}\left(\widehat{r}_{\ell}\right)+\widetilde{\delta}_{m}(\rho)
$$

Hence net profit of $m$ is:

$$
\begin{aligned}
V_{m}^{*}= & U_{m}\left(\widehat{r}_{m}\right)-\sum_{e \in \mathcal{E}} \lambda_{e}(\widehat{\mathbf{r}}) c_{e}\left(\lambda_{e}(\widehat{\mathbf{r}})\right)-\widehat{P}_{m} \\
& U_{m}\left(\widehat{r}_{m}\right)+\sum_{\ell \in \mathcal{M} \backslash\{m\}} \widetilde{U}_{\ell}\left(\widehat{r}_{\ell}\right)-\sum_{e \in \mathcal{E}} \lambda_{e}(\widehat{\mathbf{r}}) c_{e}\left(\lambda_{e}(\widehat{\mathbf{r}})\right)-\sum_{\ell \in \mathcal{M} \backslash\{m\}} \widetilde{U}_{\ell}(0)-\widehat{\delta}_{m}(\rho)
\end{aligned}
$$

By optimality of $\widehat{\mathbf{r}}$ we have

$$
\widetilde{V}_{m}-V_{m}^{*} \leq-\widetilde{\delta}_{m}(\rho)+\widehat{\delta}_{m}(\rho) \leq\left|\widetilde{\delta}_{m}(\rho)\right|+\left|\widehat{\delta}_{m}(\rho)\right|
$$

Finally, by selecting $\rho$ small enough we can guarantee $\left|\widetilde{\delta}_{m}(\rho)\right|+\left|\widehat{\delta}_{m}(\rho)\right| \leq \varepsilon$. 
We have proved that the maximum benefit user $m \in \mathcal{M}$ can attain by using some function $\widetilde{U}_{m} \in \mathbb{U}$ such that $\widetilde{U}_{m} \neq U_{m}$ goes to zero as $\rho \rightarrow 0^{+}$. Using some strategy that can not be described by some function $\widetilde{U}_{m} \in \mathbb{U}$ will be revealed by checking the activity rule, in which case a large penalty will be charged, hence being truthful is an $\epsilon$-Dominant strategy in the sense that for any user, the maximum benefit he can attain by not being truthful is $\epsilon(\rho)$ where $\epsilon(\rho) \rightarrow 0$ as $\rho \rightarrow 0^{+}$

\subsection{Conclusion}

In this chapter, we have introduced Inertial Capacity Auction, an iterative auction design for the efficient flow assignment in a network with capacity constraints and congestion externalities. The mechanism is shown to truthfully implement in $\epsilon$-Dominant strategies, the socially efficient flow configuration. In contrast to previous flow allocation mechanisms, the Inertial Capacity Auction implements a novel concept of flow inertial flow updates, which ultimately allows users internalize the effects of their collective actions and guarantees convergence even in the presence of congestion externalities. 


\subsection{Appendix of Inertial Capacity Auction}

\subsubsection{Proof of Lemma 2.1}

Suppose $\lim \sup \lambda_{e}^{t}>\bar{\lambda}_{e}$. So either $\left\{\lambda_{e}^{t}: t \geq 0\right\}$ goes to infinity (i.e. $\lambda_{e}^{t} \rightarrow \infty$ ), converges to a level greater than capacity (i.e. $\lambda_{e}^{t} \rightarrow \lambda_{e}^{*}>\bar{\lambda}_{e}$ ) or it oscillates with $\lim \sup \lambda_{e}^{t}>\bar{\lambda}_{e}$. The first two options are easily discarded since both imply $\mu_{e}^{t} \rightarrow \infty$, therefore $d_{k}^{t}=0$ for all $k \in \mathcal{K}$ with $A_{k, e}>0$ and large enough $t$, leading to $\lambda_{e}^{t} \rightarrow 0$, a contradiction. Therefore we focus on discarding the latter option of oscillating flow level $\lambda_{e}^{t}$ with $\lim \sup \lambda_{e}^{t}>\bar{\lambda}_{e}$, by contradiction:

1. Assume that $\lambda_{e}^{t}$ oscillates with $\lim \sup \lambda_{e}^{t}>\bar{\lambda}_{e}$. Therefore there exist some flow levels $X_{1}, X_{2}$ on link $e \in \mathcal{E}$ with $\bar{\lambda}_{e}<X_{1}<X_{2}<\limsup \lambda_{e}^{t}$ such that $X_{1}$ and $X_{2}$ are up-crossed and down-crossed infinitely often.

2. Let $\mathrm{T}$ be an up-crossing of $X_{2}$ and $Y(T)$ be the last up-crossing of $X_{1}$ before $T$. That is, $T$ is some $t>0$ such that $\lambda_{e}^{T-1}<X_{2} \leq \lambda_{e}^{T}$ and $Y(T)=\sup _{t \leq T}\left\{\lambda_{e}^{t-1}<X_{1} \leq \lambda_{e}^{t}\right\}$. Note that for any $T$ and $t \in[Y(T), T-1]$ we have:

- $X_{2}>\lambda_{e}^{t} \geq X_{1}>\bar{\lambda}_{e}$, therefore

- $\mu^{t+1}-\mu^{t}=\rho\left(\lambda_{e}^{t}-\bar{\lambda}_{e}\right)$

3. Now we prove that the time it takes to go from $Y(T)$ to $T$ is (unboundedly) increasing in $\mathrm{T}$. This implies that the price increase from $Y(T)$ to $T$ also grows (unboundedly) with $T$. Formally we have:

$$
\begin{aligned}
X_{2}-X_{1} & \leq \lambda_{e}^{T}-\lambda_{e}^{Y(T)-1} \\
& \leq \sum_{t=Y(T)}^{T}\left\|\lambda_{e}^{t}-\lambda_{e}^{t-1}\right\| \\
& \leq \sum_{t=Y(T)}^{T} \alpha_{t} \bar{d}_{e} \\
& \leq(\Delta T+1) \alpha_{Y(T)} \bar{d}_{e}
\end{aligned}
$$


where $\Delta T=T-Y(T)$ and $\bar{d}_{e}$ is as defined in Assumption 4. The former implies $\frac{X_{2}-X_{1}}{\alpha_{Y(T)}} \leq(\Delta T+1) \bar{d}_{e}$. Which in turn implies $\Delta T \rightarrow \infty$ as $T \rightarrow \infty$ (the time to go from $X_{1}$ to $X_{2}$ increases unboundedly with T).

Now notice that:

$$
\begin{aligned}
\mu_{e}^{T-1} & =\mu_{e}^{Y(T)}+\sum_{t=Y(T)}^{T-2}\left(\mu_{e}^{t+1}-\mu_{e}^{t}\right) \\
& =\mu_{e}^{Y(T)}+\sum_{t=Y(T)}^{T-2} \rho\left(\lambda_{e}^{t}-\bar{\lambda}_{e}\right) \\
& \geq \mu_{e}^{Y(T)}+\sum_{t=Y(T)}^{T-2} \rho\left(X_{1}-\bar{\lambda}_{e}\right) \\
& \geq \mu_{e}^{Y(T)}+\Delta T \rho\left(X_{1}-\bar{\lambda}_{e}\right)
\end{aligned}
$$

where the second and third lines come from the properties itemized on 2). Therefore the price at $T-1$ increases (unboundedly) with T. i.e. $\mu_{e}^{T-1} \rightarrow \infty$ as $T \rightarrow \infty$.

4. Suppose we select an up-crossing $T \rightarrow \infty$ therefore $\mu_{e}^{T-1} \rightarrow \infty$, assumption 3 (choking prices) implies $d_{k}^{T}=0$ for all $k \in \mathcal{K}$ with $A_{k, e}>0$. Which in turn implies $\lambda_{e}^{T}=\lambda_{e}^{T-1}\left(1-\alpha_{T}\right)<\lambda_{e}^{T-1}$. This contradicts the hypothesis that $T$ is an up-crossing of $X_{2}$ (i.e. that $\lambda_{e}^{T-1}<X_{2}<\lambda_{e}^{T}$ ).

\subsubsection{Proof of Corollary 2.2}

Let us write the price update as follows

$$
\mu_{e}^{t+1}=\mu_{e}^{t}+\rho_{t}\left(\lambda_{e}^{t+1}-\bar{\lambda}_{e}\right)
$$

where

$$
\rho_{t}= \begin{cases}\rho & \mu_{e}^{t}+\rho\left(\lambda_{e}^{t+1}-\bar{\lambda}_{e}\right)>0 \\ \frac{\mu_{e}^{t}}{\overline{\lambda_{e}}-\lambda_{e}^{t+1}} & \mu_{e}^{t}+\rho\left(\lambda_{e}^{t+1}-\bar{\lambda}_{e}\right) \leq 0\end{cases}
$$


Now let $T(t)=\inf \left\{T \geq t|| \mu_{e}^{\tau}-\mu_{e}^{*} \mid<\frac{1}{t}\right.$ for all $\left.\tau \geq T\right\}$. We can write

$$
\begin{aligned}
\left|\mu_{e}^{t}-\mu_{e}^{*}\right| & =\left|\mu_{e}^{*}-\mu_{e}^{T(t)}+\mu_{e}^{T(t)}-\mu_{e}^{t}\right| \\
& =\left|\mu_{e}^{*}-\mu_{e}^{T(t)}+\sum_{\ell=t}^{T(t)-1} \rho_{t}\left(\lambda_{e}^{\ell+1}-\bar{\lambda}_{e}\right)\right| \\
& =\frac{1}{\alpha_{t}}\left|\alpha_{t}\left(\mu_{e}^{*}-\mu_{e}^{T}(t)\right)+\alpha_{t} \sum_{\ell=t}^{T(t)-1} \rho_{t}\left(\lambda_{e}^{\ell+1}-\bar{\lambda}_{e}\right)\right|
\end{aligned}
$$

Since $\left|\mu_{e}^{t}-\mu_{e}^{*}\right| \rightarrow 0$ it follows that

$$
\left|\alpha_{t}\left(\mu_{e}^{*}-\mu_{e}^{T}(t)\right)+\alpha_{t} \sum_{\ell=t}^{T(t)-1} \rho_{t}\left(\lambda_{e}^{\ell+1}-\bar{\lambda}_{e}\right)\right| \leq L\left(\alpha_{t}\right)^{\gamma}
$$

for some $L>0$ and $\gamma>1$. Thus

$$
\begin{aligned}
\sum_{t>0} \alpha_{t}\left|\mu_{e}^{t}-\mu_{e}^{*}\right| & =\sum_{t>0}\left|\alpha_{t}\left(\mu_{e}^{*}-\mu_{e}^{T}(t)\right)+\alpha_{t} \sum_{\ell=t}^{T(t)-1} \rho_{t}\left(\lambda_{e}^{\ell+1}-\bar{\lambda}_{e}\right)\right| \\
& \leq \sum_{t>0} L\left(\alpha_{t}\right)^{\gamma}<\infty
\end{aligned}
$$

\subsubsection{Proof of Corollary 2.3}

Note from the price update rule (Eq. 2.6) that if $\lambda_{e}^{t+1}-\bar{\lambda}_{e}>0$ then $\mu_{e}^{t+1}-\mu_{e}^{t}>0$, similarly if $\lambda_{e}^{t+1}-\bar{\lambda}_{e} \leq 0$ then $\mu_{e}^{t+1}-\mu_{e}^{t} \leq 0$. Hence, $\left(\mu_{e}^{t+1}-\mu_{e}^{t}\right)\left(\lambda_{e}^{t+1}-\bar{\lambda}_{e}\right) \geq 0$ for all $t>0$. By Abel's Lemma and Corollaries 1 and 2 we have:

$$
\begin{aligned}
\sum_{t>0}\left(\mu_{e}^{t+1}-\mu_{e}^{t}\right)\left(\lambda_{e}^{t+1}-\bar{\lambda}_{e}\right) & =\sum_{t>0}\left[\left(\mu_{e}^{t+1}-\mu_{e}^{*}\right)-\left(\mu_{e}^{t}-\mu_{e}^{*}\right)\right]\left(\lambda_{e}^{t+1}-\bar{\lambda}_{e}\right) \\
& =\lim _{t \rightarrow \infty}\left[\left(\mu_{e}^{t}-\mu_{e}^{*}\right)\left(\lambda_{e}^{t+1}-\bar{\lambda}_{e}\right)\right]-\sum_{t>0}\left(\mu_{e}^{t}-\mu_{e}^{*}\right)\left(\lambda_{e}^{t+1}-\lambda_{e}^{t}\right) \\
& =\sum_{t>0}\left(\mu_{e}^{t}-\mu_{e}^{*}\right)\left(\lambda_{e}^{t}-\lambda_{e}^{t+1}\right) \\
& \leq \bar{d}_{e} \sum_{t>0} \alpha_{t}\left|\mu_{e}^{t}-\mu_{e}^{*}\right|<\infty
\end{aligned}
$$




\subsubsection{Proof of Lemma 2.2}

For all $m \in \mathcal{M}$ let us define $W_{m}: \mathbb{R}_{+} \mapsto \mathbb{R}_{+}$as follows

$$
W_{m}(p)=\max _{r \in \mathbb{R}_{+}}\left[\widetilde{U}_{m}(r)-r p_{m}\right]
$$

To highlight the fact that users demand according to $\widetilde{U}_{m}\left(\right.$ instead of $\left.U_{m}\right)$, we use $\widetilde{d}_{m}^{t}, \widetilde{r}_{m}^{t}$ and $\widetilde{P}_{m}^{t}$ (instead of $d_{m}^{t}, r_{m}^{t}$ and $\left.P_{m}^{t}\right)$. Let $\widetilde{p_{m}}(t)$ be a linear interpolation of ${\widetilde{p_{m}}}^{t}$ (i.e. $\widetilde{p_{m}}(t)={\widetilde{p_{m}}}^{\lfloor t\rfloor}+\frac{t-\lfloor t\rfloor}{\lceil t\rceil-\lfloor t\rfloor}\left({\widetilde{p_{m}}}^{\lceil t\rceil}-{\widetilde{p_{m}}}^{\lfloor t\rfloor}\right.$ ) where $\lfloor x\rfloor$ and $\lceil x\rceil$ represent the floor and ceiling functions respectively) and $\widetilde{d}_{-m}(t)$ its associated aggregate demand vector for users $\mathcal{M} \backslash\{m\}$, that is $\widetilde{d}_{-m}(t)=\sum_{\ell \in \mathcal{M} \backslash\{m\}} \widetilde{d}_{\ell}(t)$ where

$$
\widetilde{d}_{\ell}(t)=\arg \max _{r \in \mathbb{R}_{+}}\left[\widetilde{U}_{\ell}(r)-r \widetilde{p}_{\ell}(t)\right]
$$

By Envelope Theorem it follows that $\nabla W_{\ell}\left(\widetilde{p}_{\ell}(t)\right)=-\widetilde{d}_{\ell}(t)$.

After $T>0$ rounds, it holds that

$$
\widetilde{P}_{m}^{T}=-\sum_{t=0}^{T-1} \sum_{\ell \neq k} \widetilde{p}_{\ell}^{t+1}\left(\widetilde{d}_{\ell}^{t+1}-\widetilde{d}_{\ell}^{t}\right)
$$

$k \in \mathcal{K}$. By convergence, we can approximate $\widetilde{P}_{k}^{T}$ with Riemann-Stieltjes integral in the following sense:

$$
\widetilde{P}_{m}^{T}=-\sum_{\ell \neq m} \int_{1}^{T} \widetilde{p}_{\ell}(t) \partial \widetilde{d}_{\ell}(t)+\widetilde{\epsilon}_{k}^{T}(\rho)
$$

This approximation certainly introduces some error which we account in $\widetilde{\epsilon}_{k}^{T}(\rho)$, note however that as $\rho \rightarrow 0$, changes in prices and demands become infinitesimal, improving the approximation such that for any $T>0$ we have $\widetilde{\epsilon}_{k}^{T}(\rho) \rightarrow 0$ as $\rho \rightarrow 0$. 
Using integration by parts it follows that

$$
\begin{aligned}
-\int_{1}^{T} \widetilde{p}_{\ell}(t) \partial \widetilde{d}_{\ell}(t) & =-\left.\widetilde{p}_{\ell}(t) \widetilde{d}_{\ell}(t)\right|_{1} ^{T}+\int_{1}^{T} \widetilde{d}_{\ell}(t) \partial \widetilde{p}_{\ell}(t) \\
& =-\left.\widetilde{p}_{\ell}(t) \widetilde{d}_{\ell}(t)\right|_{1} ^{T}-\int_{1}^{T} \nabla W_{\ell}\left(\widetilde{p}_{\ell}(t)\right) \partial \widetilde{p}_{\ell}(t) \\
& =\left.\left\{\widetilde{p}_{\ell}(t) \widetilde{d}_{\ell}(t)-W_{\ell}\left(\widetilde{p}_{\ell}(t)\right)\right\}\right|_{1} ^{T} \\
& =-\widetilde{U}_{\ell}\left(\widetilde{d}_{\ell}^{T}\right)+\widetilde{U}_{\ell}\left(\widetilde{d}_{\ell}^{1}\right)
\end{aligned}
$$

where the second line follows from envelope theorem. Note that if the auction closes at time $T>0$, the total payment can be expressed as:

$$
\widetilde{P}_{m}^{T}+\sum_{e \in \mathcal{E}} c_{e}^{T} \lambda_{e,-m}\left(\widetilde{\mathbf{r}}^{T}\right)=-\sum_{\ell \in \mathcal{M} \backslash\{m\}} \widetilde{U}_{\ell}\left(\widetilde{d}_{\ell}^{T}\right)+\sum_{\ell \in \mathcal{M} \backslash\{m\}} \widetilde{U}_{\ell}\left(\widetilde{d}_{\ell}^{1}\right)+\sum_{e \in \mathcal{E}} c_{e}^{T} \lambda_{e,-m}\left(\widetilde{\mathbf{r}}^{T}\right)+\epsilon_{m}^{T}(\rho)
$$

Finally, $r_{m}^{t} \rightarrow \widetilde{r}_{m}$ implies $d_{m}^{t} \rightarrow \widetilde{r}_{m}$. Thus:

$$
\lim _{T \rightarrow \infty}\left[\widetilde{P}_{m}^{T}+\sum_{e \in \mathcal{E}} c_{e}^{T} \lambda_{e,-m}\left(\widetilde{\mathbf{r}}^{T}\right)\right]=-\sum_{\ell \in \mathcal{M} \backslash\{m\}} \widetilde{U}_{\ell}\left(\widetilde{r}_{\ell}\right) \quad+\sum_{\ell \in \mathcal{M} \backslash\{m\}} \widetilde{U}_{\ell}\left(\widetilde{d}_{\ell}^{1}\right)+\sum_{e \in \mathcal{E}} \lambda_{e,-m}(\widetilde{\mathbf{r}}) c_{e}\left(\lambda_{e}(\widetilde{\mathbf{r}})\right)+\delta_{m}(\rho)
$$

where

$$
\delta_{m}(\rho)=\lim _{T \rightarrow \infty} \epsilon_{m}^{T}(\rho)
$$

which finalizes the proof of this Lemma. 


\section{Chapter 3}

\section{Uplink-Temperature Inertial Auction}

\subsection{Preliminaries}

\subsubsection{Introduction}

Consider a wireless network composed of several access points (APs) and intrinsically associated users with some service quality requirements. In what follows, we shall refer to these users as primary users. Additionally consider a set of potential (secondary) users that coexist with the primary users but are not intrinsically associated with the APs.

In this chapter, we study the problem of a benevolent network manager that wants to efficiently service the secondary users without compromising the service quality of his primary users. To guarantee the service quality of primary users, the network manager sets a cap on the maximum interference secondary users can generate at each AP. This cap is known as AP temperature. For this problem, we propose Iterative Temperature Auction (ITA), an iterative auction by which the network manager can allocate the temperature of the different access points to efficiently service secondary users.

In the previous chapter (Chapter 2), we studied the efficient allocation of network capacity. In a similar way, this chapter studies the efficient use of constrained resources, namely, constrained interference power at the APs. However, structural 
differences in these problems hinder the application of the Iterative Capacity Auction (ICA), introduced in Chapter 2, to the problem at hand:

- In Chapter 2, the "network-capacity consumption" of each user can be described by a single parameter,$r_{m}$. In contrast, the "temperature consumption" of each user is vectorial (with as many components as APs).

- In the problem described in Chapter 2, each user has an intrinsic utility, derived from his individual flow rate. Externalities were assumed additive to this intrinsic utility and were assumed to be marginally symmetric (See Eq.2.1). In contrast, because of the wireless nature of the problem Chapter 3, externalities here are heavily intertwined in the utility functions.

- The differences mentioned above are reflected in the auction designs proposed. For example, the ICA (in Chapter 2) uses homogeneous prices for externalities, which are associated with congestion. In contrast, the $I C A$ uses discriminative (i.e. user specific) prices for externalities, which are associated with interference power. Furthermore, since externalities depend on the functional form of the utilities, the auctioneer needs to directly request some extra information, that cannot be extracted from the power profile updates.

\subsubsection{Basic Framework}

Assume the access point association and power profiles are fixed for the primary users. Configuration of the uplink access of the secondary users is to be assessed, constrained by some restrictions on the quality of the primary users. The system access method is CDMA DSSS, in which each user is assigned a code that allows several users to share the same bandwidth.

Formally, let $\mathcal{A}=\{1,2, \ldots, A\}$ and $\mathcal{M}=\{1,2, \ldots, M\}$ be the index sets of access points and secondary users respectively. Note that $M$ will denote the number of secondary users, and $A$ the number of APs. 
To guarantee the service quality of the primary users, the interference generated by the secondary users at the AP $a \in \mathcal{A}$ must be no greater than a threshold $\bar{P}_{a}$. This cap is known as the interference temperature of the AP. Note that the set of primary users is not indexed; all information we require to assess the power profile of secondary users is the temperature on each AP. For simplicity, we assume all APs have unitary and mutually exclusive bandwidth. The results are easily extended to the case of different bandwidths.

Let $\widehat{p}_{m, a}$ be the transmission power of the signal from user $m \in \mathcal{M}$ to AP $a \in \mathcal{A}$, and $h_{m, a}$ the associated gain. Thus $p_{m, a}=\left|h_{m, a}\right|^{2} \widehat{p}_{m, a}$ is the power user $m \in \mathcal{M}$ generates at the $\operatorname{AP} a \in \mathcal{A}$. Let $\mathbf{p}_{m}=\left[p_{m, 1}, p_{m, 2}, \ldots, p_{m, A}\right]$ denote user $m$ 's power profile. Note that in contrast with most literature, our power profile refers to the power at the receiver (not at the transmitter), this will certainly ease the notation that follows. Due to the economic implications of our analysis, it is convenient to think of $\bar{P}_{a}$ as a power level to be consumed by secondary users and $\mathbf{p}_{m}$ as the power-consumption profile of $m \in \mathcal{M}$.

Let $\mathbf{p}=\left[\mathbf{p}_{1}, \mathbf{p}_{2}, \ldots, \mathbf{p}_{M}\right]$ denote the joint power profile of all users. The aggregate power of secondary users at the $\mathrm{AP} a \in \mathcal{A}$, is denoted by $P_{a}(\mathbf{p})=\sum_{m \in \mathcal{M}} p_{m, a}$. The rate achieved by secondary user $m \in \mathcal{M}$ will be a function of the joint power profile p, as follows:

$$
r_{m}(\mathbf{p})=\sum_{a \in A} \log _{2}\left(1+\frac{p_{m, a}}{N_{a}+G\left(I_{a}+\sum_{i \neq m} p_{i, a}\right)}\right)
$$

where: (i) $G<1$ is the system gain, which is given by the coding of the CDMA DSSS system, (ii) $N_{a}$ is the noise power at the AP $a \in \mathcal{A}$, and (iii) $I_{a}$ is the power received from the primary users [11].

Let $U_{m}\left(r_{m}\right)$ be $m$ 's quasilinear utility (willingness to pay), when achieving aggregate rate $r_{m} \geq 0$. We assume $U_{m}: \mathbb{R}_{+} \rightarrow \mathbb{R}_{+}$is strictly increasing and concave. Concavity of $U_{m}()$ is a very reasonable assumption. If user $m \in \mathcal{M}$ is given a small rate $r_{m} \geq 0$ he will accommodate the information that provides 
the maximum utility per data rate (information with the highest priority); as the data rate increases he can start to accommodate information that provides lower marginal utility (information with lower priority). Therefore, it is reasonable to consider a concave utility function in order to capture this decreasing marginal utility.

The social surplus achieved by a joint power profile $\mathbf{p} \in \mathbb{R}_{+}^{M A}$ is $\sum_{m \in \mathcal{M}} U_{m}\left(r_{m}(\mathbf{p})\right)$. A joint power profile is said to be (socially) efficient if it maximizes the social surplus. That is, if it solves the following maximization problem:

$$
\arg \max _{\mathbf{p} \in \mathcal{P}}\left[\sum_{m \in \mathcal{M}} U_{m}\left(r_{m}(\mathbf{p})\right)\right]
$$

where $\mathcal{P}$ is the set of feasible power profiles, i.e. $\mathcal{P}=\left\{\mathbf{p} \in \mathbb{R}_{+}^{M A}: P_{a}(\mathbf{p}) \leq\right.$ $\left.\bar{P}_{a}, \quad \forall a \in \mathcal{A}\right\}$.

In what follows we assume concavity of the social surplus $\sum_{m \in \mathcal{M}} U_{m}\left(r_{m}(\mathbf{p})\right)$. Hence, sufficient conditions for efficiency of $\mathbf{p} \in \mathcal{P}$ are:

$$
\begin{gathered}
U_{m}^{\prime}\left(r_{m}(\mathbf{p})\right) \frac{\partial r_{m}}{p_{m, a}}(\mathbf{p})-\sum_{i \neq m} U_{i}^{\prime}\left(r_{i}(\mathbf{p})\right) \frac{\partial r_{i}}{p_{m, a}}(\mathbf{p})-\mu_{a} \leq 0 \\
\text { for all } m \in \mathcal{M}, a \in \mathcal{A} \text { with equality if } p_{m, a}>0
\end{gathered}
$$

and

$$
\mu_{a}\left(\sum_{m \in \mathcal{M}} p_{m, a}-\bar{P}_{a}\right)=0 \quad \text { for all } a \in \mathcal{A}
$$

for some $\mu_{a} \geq 0$ with $a \in \mathcal{A}$.

Let us highlight some insights of the problem we described above. The temperature of each AP is a divisible and heterogeneous commodity. Divisible, because users can use fractions of the available temperatures. Heterogeneous, because the benefit a secondary user attains from "consuming some of level of temperature", changes from one AP to another.

The power allocation problem, described above, needs to account for negative 
externalities with some level of complexity. The higher the power of one user at a given access point, the higher his data rate. However, this will also erode the data rate of other users affiliated to the same A.P. In comparison with the flow adjustment problem, in Chapter 2, externalities here are not homogeneous nor additive to the intrinsic utility. This characteristics, will change the definition of the externality price, and the way payments are collected. We will also need some information feedback from users, not only their profile update.

Our objective is to design an iterative auction that drives the system to the efficient configuration. The main difficulty in this design is that secondary users are rational bidders with private information. That is, they are willing to maximize their individual utility, even at the expense of the social surplus. Hence, the need to align individual incentives with the social objective.

In order to provide some intuition in the auction design we introduce later, let us make some important observations. Suppose the auctioneer (primary network) knows the efficient joint power profile $\mathbf{p}^{*} \in \mathbb{R}_{+}^{M A}$, shadow prices $\left\{\mu_{a} \geq 0: a \in \mathcal{A}\right\}$ and marginal utilities $\left\{U_{m}^{\prime}\left(r_{m}\left(\mathbf{p}^{*}\right)\right)\right\}$. In this case, each access point $a \in \mathcal{A}$ can calculate the following discriminative prices for each user $m \in \mathcal{M}$

$$
\begin{aligned}
\zeta_{a, m} & =\sum_{i \neq m}\left[U_{i}^{\prime}\left(r_{i}\left(\mathbf{p}^{*}\right)\right) \frac{\partial r_{i}}{\partial p_{m, a}}\left(\mathbf{p}^{*}\right)\right] \\
& =\sum_{i \neq m} U_{i}^{\prime}\left(r_{i}\left(\mathbf{p}^{*}\right)\right)\left(\frac{G p_{i, a}^{*}}{\left[p_{i, a}^{*}+N_{a}+G\left(I_{a}+\sum_{j \neq i} p_{j, a}^{*}\right)\right]\left[N_{a}+G\left(I_{a}+\sum_{j \neq i} p_{j, a}^{*}\right)\right]}\right) \log _{2} e
\end{aligned}
$$

Now, assume m's competitors have power profiles fixed at their efficient levels, $\left\{\mathbf{p}_{i}^{*} \in \mathbb{R}_{+}^{A}: i \in \mathcal{M} \backslash\{m\}\right\}$. Let $\mathbf{p}_{-m}^{*}$ represent the efficient profile of $m$ 's competitors, and $r_{m}\left(\mathbf{p}_{m}, \mathbf{p}_{-m}^{*}\right)$ be the rate $m$ would achieve with power profile $\mathbf{p}_{m}$.

If each AP $a \in \mathcal{A}$, imposes fixed prices $\mu_{a}$ and $\zeta_{a, m}$, to user $m \in \mathcal{M}$; then, user $m$ faces the following problem:

$$
\max _{\mathbf{p}_{m} \in \mathbb{R}_{+}^{A}}\left[U_{m}\left(r_{m}\left(\mathbf{p}_{m}, \mathbf{p}_{-m}^{*}\right)\right)-\sum_{a \in \mathcal{A}} p_{m, a}\left(\mu_{a}+\zeta_{m, a}\right)\right]
$$


By concavity, user $m$ will set his power profile to some $\mathbf{p}_{m} \in \mathbb{R}_{+}^{A}$, such that

$$
U_{m}^{\prime}\left(r_{m}\left(\mathbf{p}_{m}, \mathbf{p}_{-m}^{*}\right)\right) \frac{\partial r_{m}}{p_{m, a}}\left(\mathbf{p}_{m}, \mathbf{p}_{-m}^{*}\right)-\zeta_{m, a}-\mu_{a} \leq 0
$$

for all $a \in \mathcal{A}$ with equality if $p_{m, a}>0$. Which resembles condition in Eq. (3.3.a), and uniquely defines $\mathbf{p}_{m}^{*}$. The analysis above inspires the Inertial Temperature Auction, which is formally introduced in Section 3.3.

The Inertial Temperature Auction runs in discrete time steps (or rounds) indexed by $t \in\{0,1,2, \ldots\}$. At each round, each secondary user $m \in \mathcal{M}$ is given some discriminative prices at each AP, and is asked to update his power profile following a predefined rule (similar to the one above). Users that follow this rule, are called truthful. Note that users may not be truthful, and respond to this request following their own strategy. However, the maximum benefit any user can attain by not being truthful, can be made as small as desired. In other words, being truthful is an $\epsilon$-dominant strategy. Furthermore, when all users are truthful the socially efficient power profile is implemented. In the context of mechanism design theory, we say that ITA truthfully implements the socially efficient power profile, in $\epsilon$-dominant strategies.

\subsection{Literature Review}

In order to implement the socially efficient power configuration, the primary network manager can use the VCG mechanism. However this mechanism has many drawbacks, already mentioned in Section 2.2. The main drawbacks of VCG are related to the complete revelation of the utility-structures and the demands for sophisticated computation infrastructure $[3,30]$. In order to circumvent many of these difficulties, several iterative auction designs have been proposed.

Ausubel proposes an auction for heterogeneous divisible resources that implements the efficient resource configuration in Nash equilibrium [2]. However, Ausubel's auction does not account for externalities. To explore the results of 
naively applying Ausubel's auction in our problem, consider the following example. Two secondary users, with utilities $U_{m}\left(r_{m}\right)=2 r_{m}$ for $m \in\{1,2\}$, want to establish an up-link connection with an AP that has an available temperature of $T=10$, Interference from primary users is $I=5$, system gain is $G=1 / 1024$ and noise power is $N=1$. In the Annex, Section 3.8.1, we prove that the objective function in hand is concave. A discrete time implementation of Ausubel's design, can be as follows: Let $\mu^{t}$ be the price for power received at the AP, and $p_{m}^{t}$ the power received from secondary user $m \in\{1,2\}$ at time $t>0$. Assuming truthful users, at each iteration $t>0$, each user $m \in\{1,2\}$ updates his power profile as follows

$$
p_{m}^{t}=\arg \max _{d \in \mathbb{R}_{+}}\left[U_{m}\left(r_{m}\left(d, p_{i}^{t-1}\right)\right)-d \mu^{t-1}\right]
$$

where $r_{m}\left(d, p_{i}^{t-1}\right)$ denotes the data rate user $m$ achieves with power $d$, if the power of his competitor $i \neq m$ remains unchanged. In turn, the AP manager updates the price as follows:

$$
\mu^{t}=\left[\mu^{t-1}+\rho_{t}\left(\sum_{m \in\{1,2\}} p_{m}^{t}-T\right)\right]^{+}
$$

where $\rho_{t}=\frac{1}{t+1}$ and $[x]^{+}=\max \{0, x\}$.

However, this implementation of Ausubel's design, induces the cyclical behavior in Fig. 3.1. By the arguments introduced in Section 3.1.2, discriminative prices $\zeta_{m}^{t}$, as described in Eq.(3.5), will align truthful power updates with the efficiency.

$$
\zeta_{m}^{t}=U_{i}^{\prime}\left(r_{i}\left(p_{i}^{t}, p_{m}^{t}\right)\right)\left(\frac{G p_{i}^{t}}{\left[p_{i}^{t}+N+G\left(I+p_{m}^{t}\right)\right]\left[N+G\left(I+p_{m}^{t}\right)\right]}\right) \log _{2} e
$$

In this case we say that user $m \in\{1,2\}$ is truthful if he updates his power profile according to:

$$
p_{m}^{t}=\arg \max _{d \in \mathbb{R}_{+}}\left[U_{m}\left(r_{m}\left(d, p_{i}^{t-1}\right)\right)-d\left(\mu^{t-1}+\zeta_{m}^{t-1}\right)\right]
$$




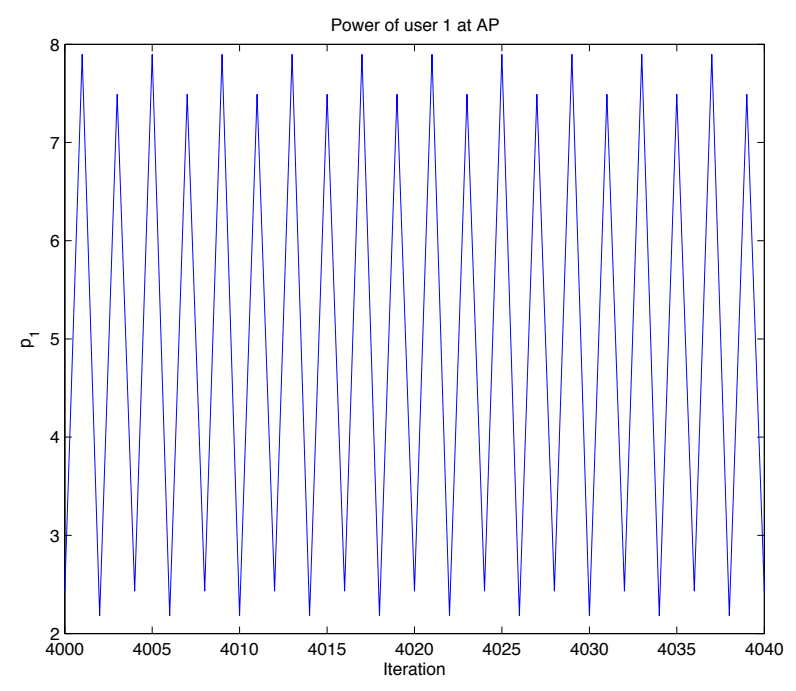

Figure 3.1

with this additional prices $\left(\left\{\zeta_{m}\right\}_{m \in\{1,2\}}\right)$, we say that truthful updates are aligned with efficiency because convergence of the joint power profile implies efficiency conditions in Eq. (3.3.a). Unfortunately when these prices are introduced, convergence is not achieved and results are virtually the same as those in Fig. 3.1. Several authors have studied auction designs specifically designed for the efficient use of wireless resources $[5,7,14,29,31,36]$.

Boche and Naik [7] make a general approach to the design and implementation of mechanisms for the allocation of resources in interference coupled wireless systems. Their work focuses on the conditions for implementation of different system configurations in Nash equilibrium and dominant strategies. This work reveals some inherent difficulties in the strategy proof (i.e. truthful) implementation of socially efficient configurations and characterizes Pareto optimal bounds for different constraints in the power configuration (namely total power and individual power constraint).

Bae et al. [5], use the Ausubel's auction to sell a temperature $T$ of a primary receiver to several transmitters receivers (tx-rx) pairs. They assume interference from the unique primary user is much larger than the interference from any secondary user and modify Ausubel's auction to achieve a core-selecting auction (not necessarily efficient). Our problem can be easily transformed in the framework 
stated by Bae et al. if we consider the AP to be the receiver of the primary user and having collocated receivers for the tx-rx pairs. However our mechanism allows for multiple APs and we do not assume the power from any secondary user to be negligible compared to the primary users'. In fact note that such approach may not be practical for the dynamic use of spectrum by technologies having complementary busy hours as is the case of cellular telephony and digital video broadcast [29].

$\mathrm{Yu}$ et al. [36], propose a pricing scheme for power control in a framework where one access point tries to maximize its revenue by selling a residual power to secondary users. However utility of secondary users is assumed to be of the type $U_{m}=\alpha_{m} r_{m}$ and users are assumed to be price takers.

Huang et al. [14], study auction mechanisms for sharing spectrum with secondary users given a maximum interference temperature. In this work only one measuring point is considered and efficiency is attained for what the authors call large systems (where available bandwidth, temperature and the number of secondary suers go to infinity), furthermore this configuration is achieved in Nash equilibrium and with some restrictions in the functional form of secondary users' utilities.

Mohammadian and Abolhassani [31], consider a scenario with several primary users with different receivers (hence considering interference temperature at several sites, in contrast with Huang et al.[14]). This work defines an oligopoly market that clears in a Nash Equilibrium. However efficiency is not guaranteed and a specific utility functional form is assumed.

In what follows we introduce the Inertial Temperature Auction (ITA), an iterative auction design that implements (in $\epsilon$-dominant strategies) the socially efficient joint power profile.

This chapter is organized as follows; in Section 3.3 we propose ITA, Section 3.4 introduces some basic assumptions, Sections 3.5 and 3.6 prove that ITA truthfully implements the efficient joint power profile in $\epsilon$-Dominant strategies. Finally section 
3.7 presents some conclusions and important remarks.

\subsection{Inertial Temperature Auction}

The Inertial Temperature Auction runs in discrete time rounds $t=\{1,2, \ldots\}$ where each $\mathrm{AP}(a \in \mathcal{A})$ reveals a common control price $\mu_{a}^{t}$ and a discriminatory externality price $\zeta_{a, m}^{t}$ for each secondary user $m \in \mathcal{M}$. For any $\operatorname{AP} a \in \mathcal{A}$, control prices $\left\{\mu_{a}^{t}\right\}_{t \geq 0}$ will guarantee that the aggregate power from secondary users is below the threshold temperature $\bar{P}_{a}$. For any user $m \in \mathcal{M}$, externality prices $\left\{\zeta_{a, m}^{t}\right\}_{a \in \mathcal{A}, t \geq 0}$ will help $m$ internalize the externality effect imposed on his competitors. Given such prices, each user $m \in \mathcal{M}$ calculates his demand profile $\mathbf{d}_{m}^{t}=\left[d_{m, 1}^{t}, \ldots, d_{m, A}^{t}\right]$ following a predefined rule (see Eq. 3.6), however the power profile is not updated to current demand, it is updated towards demand with an increasing inertia that prevents abrupt changes in the joint power profile (details will be given later). Note that we used $\mathbf{d}_{m}^{t}$ to denote demand profile of user $m \in \mathcal{M}$ at time $t \geq 0$, while his power profile is denoted by $\mathbf{p}_{m}^{t}=\left[p_{m, 1}^{t}, \ldots, p_{m, A}^{t}\right]$. Joint power profile is denoted by $\mathbf{p}^{t}=\left[\mathbf{p}_{1}^{t}, \ldots, \mathbf{p}_{M}^{t}\right]$ and joint demand profile by $\mathbf{d}^{t}=\left[\mathbf{d}_{1}^{t}, \ldots, \mathbf{d}_{M}^{t}\right]$. Each AP will in turn update prices following some simple rules (see Eq.3.8 and Eq.3.9). The process is repeated until a stationarity criteria is achieved. Let $\boldsymbol{\mu}^{t}=\left[\mu_{1}^{t}, \ldots, \mu_{A}^{t}\right]$, the auction finishes when the stationarity criteria, $\left|\mathbf{d}^{t}-\mathbf{p}^{t-1}\right|+\left|\boldsymbol{\mu}^{t}-\boldsymbol{\mu}^{t-1}\right|<\eta$ is achieved for some small $\eta>0$. The following pseudocode, formally introduces the Inertial Temperature Auction:

1. General information. Users are informed about the gain of the system (i.e. $G)$ and the interference from primary users at each AP (i.e. $\left\{I_{a}\right\}_{a \in \mathcal{A}}$ )

2. Initialization. Prices and allocations are initialized as follows: $p_{m, a}^{0}=d_{m, a}^{0}=$ $0, \zeta_{a, m}^{0}=0$ and $\mu_{a}^{0}=\bar{\mu}$ for all $a \in \mathcal{A}$ and $m \in \mathcal{M}$, where $\bar{\mu}$ is a price large enough to guarantee null demand from each user to each AP ( $\bar{\mu}$ is formally introduced in Section 3.4). 
3. Iterate. For $t>0$, power profiles and prices are updated:

(a) Each access point $(a \in \mathcal{A})$ reveals the aggregate power from secondary users $\left(P_{a}^{t-1}=\sum_{m \in \mathcal{M}} p_{m, a}^{t-1}\right)$, common price $\left(\mu_{a}^{t-1}\right)$, and discriminatory prices $\left(\left\{\zeta_{a, m}^{t-1}\right\}_{m \in \mathcal{M}}\right)$ from the previous iteration.

(b) Each secondary user $m \in \mathcal{M}$ finds his demand profile as follows

$$
\mathbf{d}_{m}^{t}=\arg \max _{\mathbf{d}_{m} \in \mathbb{R}_{+}^{A}}\left[U_{m}\left(r_{m}\left(\mathbf{d}_{m}, \mathbf{p}_{-m}^{t-1}\right)\right)-\mathbf{d}_{m} \cdot\left(\boldsymbol{\mu}^{t-1}+\boldsymbol{\zeta}_{m}^{t-1}\right)\right]
$$

where $\boldsymbol{\zeta}_{m}^{t-1}=\left[\zeta_{1, m}^{t-1}, \ldots, \zeta_{A, m}^{t-1}\right]$ and $r_{m}\left(\mathbf{d}_{m}, \mathbf{p}_{-m}^{t-1}\right)$ denotes the data rate user $m \in \mathcal{M}$ will achieve with power profile $\mathbf{d}_{m}$ if the power profile of his competitors $\mathcal{M} \backslash\{m\}$ remains unchanged. In what follows we shall refer to the demand defined in Eq. 3.6 as the truthful demand.

(c) Users update their power profile as follows:

$$
\mathbf{p}_{m}^{t}=\mathbf{p}_{m}^{t-1}+\alpha_{t}\left(\mathbf{d}_{m}^{t}-\mathbf{p}_{m}^{t-1}\right)
$$

for each $m \in \mathcal{M}$, where $\alpha_{t} \in(0,1)$ and $\alpha_{t} \rightarrow 0^{+}$(more details on $\alpha_{t}$ in Section 3.3.3).

(d) After power profiles have been updated each secondary user $m \in \mathcal{M}$ reports $U_{i}^{\prime}\left(r_{i}\left(\mathbf{p}^{t}\right)\right)$. In response to this report and the power profile updates, each access point $a \in \mathcal{A}$ updates his control and externality prices as follows:

$$
\mu_{a}^{t}=\left[\mu_{a}^{t-1}+\rho\left(P_{a}^{t}-\bar{P}_{a}\right)\right]^{+}
$$

where $\rho>0$, and

$$
\zeta_{a, m}^{t}=\sum_{i \neq m}\left(\frac{U_{i}^{\prime}\left(r_{i}\left(\mathbf{p}^{t}\right)\right) G p_{i, a}^{t}}{\left[p_{i, a}^{t}+N_{a}+G\left(I_{a}+\sum_{j \neq i} p_{j, a}^{t}\right)\right]\left[N_{a}+G\left(I_{a}+\sum_{j \neq i} p_{j, a}^{t}\right)\right]}\right) \log _{2} e
$$


(e) The auctioneer checks the stopping rule $\left|\mathbf{d}^{t}-\mathbf{p}^{t-1}\right|+\left|\boldsymbol{\mu}^{t}-\boldsymbol{\mu}^{t-1}\right|<\eta$. If the condition does not hold, iterate again (steps a) through e)), if it does, the auction ends and user $m \in \mathcal{M}$ is assessed a payment of $\tau_{m}^{t}$ defined by

$$
\tau_{m}^{t}=\tau_{m}^{t-1}-\sum_{i \neq m}\left[r_{i}\left(\mathbf{p}^{t}\right)-r_{i}\left(\mathbf{p}^{t-1}\right)\right] U_{i}^{\prime}\left(r_{i}\left(\mathbf{p}^{t}\right)\right)
$$

with $\tau_{m}^{0}=0$ for all $m \in \mathcal{M}$. Note that this payment can be collected throughout the iterations. In subsection 3.3.4 we propose a device for collecting this payment in an iterative and distributed fashion. However the auctioneer may use a centralized entity or any other device he finds convenient.

\subsubsection{Notes about the auction design}

We would like to highlight the following facts of the algorithm:

- In step 3.a, each discriminatory price need only be revealed to the respective secondary user.

- In step 3.b when user $m \in \mathcal{M}$ calculates his truthful demand as stated in (Eq. 3.6) he does not need to know the previous power profile of his competitors, having the aggregate power of all APs (i.e. $\left\{P_{a}^{t-1}\right\}_{a \in \mathcal{A}}$ ) will be enough.

- Note that users don't report their demand directly to APs however this will be indirectly revealed by the change in the power profile.

- Users that update their power profile according to Equations (3.6) and (3.7) and truthfully report $U_{m}^{\prime}\left(\mathbf{p}^{t}\right)$ are said to be truthful. Note that given the private nature of the utility function $U_{m}()$, users may deviate from this desired behavior. However in Section 3.6 we prove that it is in the best interest of each user to follow these rules, no matter the strategies followed by his competitors. 
- When applying the ITA to the problem of studied in the literature review (Section 3.2), the received power of user 1 at the AP is as shown in Fig. 3.2. The power for user 2 is virtually the same. Note that the efficient configuration in which each user receives half of the available temperature $(T=10)$ is achieved.

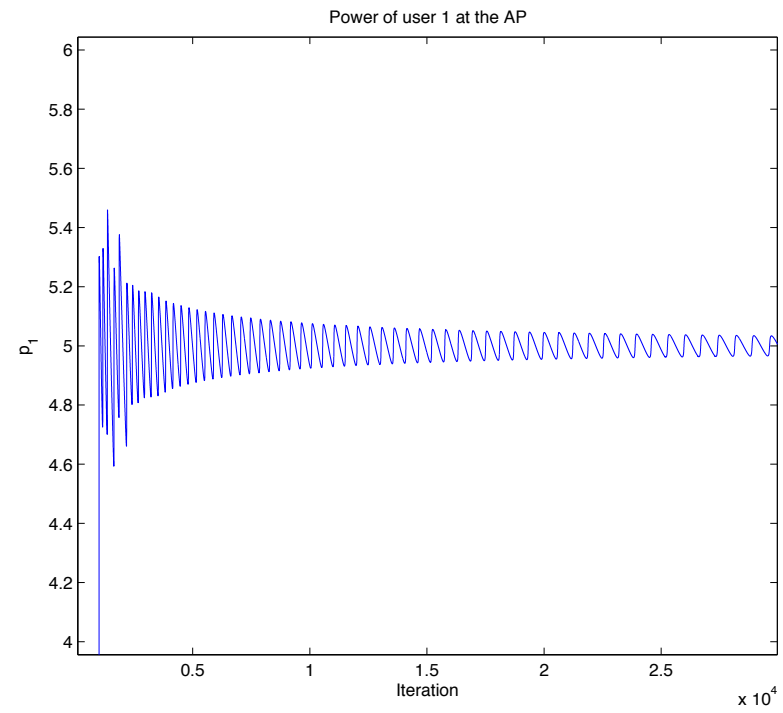

Figure 3.2

\subsubsection{About externality price}

The externality price $\left(\zeta_{a, m}^{t}\right)$ will help user $m \in \mathcal{M}$ internalize the effect of $p_{m, a}$ on his competitors utility given joint profile $\mathbf{p}^{t}$. We now provide an intuitive explanation of $\zeta_{a, m}^{t}$, the formal proof of these arguments is given in the following sections. Note that

$$
\zeta_{a, m}^{t}=-\sum_{i \neq m}\left[U_{i}^{\prime}\left(r_{i}\left(\mathbf{p}^{t}\right)\right) \frac{\partial r_{i}}{\partial p_{m, a}}\left(\mathbf{p}^{t}\right)\right]
$$

Furthermore truthful demand implies

$$
U_{m}^{\prime}\left(r_{m}\left(\mathbf{d}_{m}^{t+1}, \mathbf{p}_{-m}^{t}\right)\right) \frac{\partial r_{m}}{\partial p_{m, a}}\left(\mathbf{d}_{m}^{t+1}, \mathbf{p}_{-m}^{t}\right)-\left(\mu_{a}^{t}+\zeta_{m, a}^{t}\right) \leq 0
$$

with equality if $d_{m, a}^{t+1}>0$ 
Hence if $\mathbf{d}^{t} \rightarrow \mathbf{p}^{*}, \mathbf{p}^{t} \rightarrow \mathbf{p}^{*}$ and $\boldsymbol{\mu}^{t} \rightarrow \boldsymbol{\mu}^{*}$ for some $\mathbf{p}^{*} \in \mathbb{R}_{+}^{M A}, \boldsymbol{\mu}^{*} \in \mathbb{R}_{+}^{A}$, the limit configuration $\left(\mathbf{p}^{*}\right)$ will hold efficiency condition in Eq. 3.3.a.

\subsubsection{About inertia}

We state the following conditions on the schedule $\left\{\alpha_{t}\right\}_{t \geq 0}$

- $\alpha_{t} \in(0,1)$

- $\alpha_{t} \rightarrow 0^{+}$with $\alpha_{t+1}>\alpha_{t}$ for $t \geq 0$.

- $\sum_{t \geq 0} \alpha_{t} \rightarrow \infty$

- $\sum_{t \geq 0}\left(\alpha_{t}\right)^{\gamma}<\infty$ for any $\gamma>1$

Note that since $\alpha_{t} \rightarrow 0^{+}$power adjustment is increasingly less responsive to demand. However since $\sum_{t \geq 0} \alpha_{t} \rightarrow \infty$ any user can make sure its power profile approximates any desired value. In other words, users do have control on their power profile, however this control is increasingly more difficult as the auction progresses. This inertia in the change of the joint power profile allows users to internalize the effect of collective updates, preventing the erratic behavior we found applying other mechanisms that are not designed to deal with externalities (see Fig. 3.1).

\subsubsection{About payment rule}

When introducing the payment rule (See Eq. 3.10) it is not clear how this payments are to be implemented. We leave this as an open problem to the auctioneer (i.e. the primary network administrator). However we would like to highlight that this aggregate payment can be implemented in an iterative and distributed schedule where the administrator of AP $a \in \mathcal{A}$ charges user $m \in \mathcal{M}$ after iteration $t>0$ a payment of $\Delta \tau_{m, a}^{t}=-\sum_{i \neq m}\left(r_{i, a}^{t}-r_{i, a}^{t-1}\right) U_{i}^{\prime}\left(r_{i}^{t}\right)$ where $\left(r_{i, a}^{t}\right.$ is the rate user $m$ achieves at $\mathrm{AP} a$ at time $t$, i.e.: 


$$
r_{i, a}^{t}=\log _{2}\left(1+\frac{p_{i, a}^{t}}{N_{a}+G\left(I_{a}+\sum_{j \neq i} p_{j, a}^{t}\right)}\right)
$$

and $\left(r_{i, a}^{t}-r_{i, a}^{t-1}\right)$ is the change in rate of $m$ at AP $a$ after iteration $t$. the administrator of AP $a \in \mathcal{A}$

Let us give some intuition about the payment rule in Eq. 3.10), at iteration $t$ user $m$ will pay $\Delta \tau_{m, a}^{t}=-\sum_{i \neq m}\left(r_{i}^{t}-r_{i}^{t-1}\right) U_{i}^{\prime}\left(r_{i}^{t}\right)$, which approximates the negative change in utility for $m$ 's competitors after iteration $t>0$, hence the aggregate payment $\tau_{m}$ will approximate the negative of the change in his competitors utility since the beginning of the auction until the end. Hence if the joint power profile converges (i.e. $\mathbf{p}^{t} \rightarrow \mathbf{p}$ for some $\mathbf{p} \in \mathbb{R}_{+}^{M A}$ ), which we prove in Theorem 2.2, the aggregate payment of $m$ will be:

$$
\tau_{m} \approx-\sum_{i \neq m}\left[U_{i}\left(r_{i}\left(\mathbf{p}^{*}\right)\right)-U_{i}\left(r_{i}\left(\mathbf{p}^{0}\right)\right)\right]
$$

Therefore profit of user $\mathrm{m}$ can be calculated as a function of the limit joint power profile $\left(\mathbf{p}^{*}\right)$ as follows

$$
\begin{aligned}
V_{m}\left(\mathbf{p}^{*}\right) & \approx U_{m}\left(r_{m}\left(\mathbf{p}^{*}\right)\right)-\tau_{m} \\
& \approx \sum_{j \in \mathcal{M}} U_{j}\left(r_{j}\left(\mathbf{p}^{*}\right)\right)-\sum_{i \neq m} U_{i}\left(r_{j}\left(\mathbf{p}^{0}\right)\right)
\end{aligned}
$$

Note that the term $\sum_{i \neq m} U_{i}\left(r_{j}\left(\mathbf{p}^{0}\right)\right)$ is a constant in what refers to the strategy of user $m$. Therefore $m$ 's final profit will be a function of the final power profile $\left(\mathbf{p}^{*}\right)$, furthermore his profit is the social surplus up to a constant. Hence it is in the best interest of $m$, to achieve the final configuration that maximizes the social surplus (i.e. the socially efficient configuration).

In the following sections we prove the three main properties of our auction design. In Theorem 3.1 we prove the convergence of control prices $\mu_{a}^{t}$ for all $a \in \mathcal{A}$ under the assumption of truthful power update. In Theorem 3.2 (also 
under the assumption of truthful power updates) we prove that the joint power profile $\mathbf{p}^{t}$ converges to the unique efficient profile (the solution of problem in Eq. 3.2). Finally, in Theorem 3.3 we prove that it is in the best interest of users $m \in \mathcal{M}$ to update truthfully, independent of the behavior of his competitors. Note that the rationality behind most of these results has been already outlined in the ("intuitive") introduction of the externality price and payment rule. For the formal prove we require the following set of assumptions.

\subsection{Assumptions}

The standing assumptions for the analysis of the proposed auction design are as follows:

Assumption 1: For each user $m \in \mathcal{M}, U_{m}\left(r_{m}\right)$ is twice continuously differentiable and strongly concave in $r_{m}$.

Assumption 2: The social surplus $\sum_{m \in M} U_{m}\left(r_{m}(\mathbf{p})\right)$ is strictly concave in $\mathbf{p}$.

Assumption 3: There exist some $\bar{\mu}>0$ such for any AP with a (per powerunit) price larger than $\bar{\mu}$, the power demand of all secondary users is always zero. We should refer to $\bar{\mu}$ as the "choking" price. ${ }^{1}$

Assumption 4: Each user $m \in \mathcal{M}$ has a maximum power demand at each AP $a \in \mathcal{A}$, say $\bar{d}_{m, a}>0$, such that if $\mathbf{p}^{*}$ is the solution to problem in Eq. 3.2 then $d_{m, a}^{*}<\bar{p}_{m, a}$. Note this also implies that the norm of any joint demand and power profile is bounded. i.e. there exist some $\bar{d}$ such that $\left|\mathbf{p}^{t}\right|<\bar{d}$ and $\left|\mathbf{d}^{t}\right|<\bar{d}$ for all $t \geq 0$.

\footnotetext{
${ }^{1}$ For a formal description of Assumption 3, let $\mathbf{p}_{m,-a} \in \mathbb{R}_{+}^{A-1}$ represent the power of user $m \in \mathcal{M}$ at all APs but $a \in \mathcal{A}$ and $\mathbf{p}_{-m} \in \mathbb{R}_{+}^{(M-1) A}$ the power profile of $m$ 's competitors. Also let $r_{m}\left(d, \mathbf{p}_{m,-a}, \mathbf{p}_{-m}\right)$ the rate of user $m$ when $p_{m, a}=d$ and the rest of the joint power profile is as described by $\mathbf{p}_{m,-a}$ and $\mathbf{p}_{-m}$. Assumption 3 states that
}

$$
\arg \max _{d \in \mathbb{R}_{+}}\left[U_{m}\left(r_{m}\left(d, \mathbf{p}_{m,-a}, \mathbf{p}_{-m}\right)\right)-d \mu_{a}\right]=0
$$

for all $a \in \mathcal{A}, \mathbf{p}_{m,-a} \in \mathbb{R}_{+}^{A-1}, \mathbf{p}_{-m} \in \mathbb{R}_{+}^{(M-1) A}$, and $\mu_{a}>\bar{\mu}$ 


\subsection{Convergence of Prices and Joint Power Profile}

In this section we study the stability and efficiency of the proposed mechanism assuming that all users update their demand truthfully, that is $\mathbf{p}_{m}^{t}=\mathbf{p}_{m}^{t-1}+\alpha_{t}\left(\mathbf{d}_{m}^{t}-\right.$ $\mathbf{p}_{m}^{t-1}$ ) where $\mathbf{d}_{m}^{t}$ is as stated in Eq. 3.6 for all $m \in \mathcal{M}$.

Lemma 3.1 (Boundedness of secondary power $P_{a}^{t}=\sum_{m \in \mathcal{M}} p_{m, a}^{t}$ ). $\limsup _{t \rightarrow \infty} P_{a}^{t} \leq$ $\bar{P}_{a}, a \in \mathcal{A}$.

Proof: For the formal proof we refer the reader to the appendix. In what follows, we provide a sketch of the proof. Suppose $P_{a}^{t} \rightarrow \infty$ or $P_{a}^{t} \rightarrow P_{a}^{*}>\bar{P}_{a}$, this implies $\mu_{a}^{t} \rightarrow \infty$, which in turn implies $d_{m, a}^{t} \rightarrow 0$ for all $m \in \mathcal{M}$, therefore $P_{a}^{t} \rightarrow 0$,

a contradiction. Now assume $P_{a}^{t}$ oscillates in the limit (i.e. $\lim _{\sup _{t \rightarrow \infty}} P_{a}^{t}>$ $\left.\liminf \operatorname{in}_{t \rightarrow \infty} P_{a}^{t}\right)$ with $\lim \sup _{t \rightarrow \infty} P_{a}^{t}>\bar{P}_{a}$. This implies the existence of an interval $\left[X_{1}, X_{2}\right]$ with $\bar{P}_{a}<X_{1}<X_{2}$ which is up-crossed infinitely often. Because of increasing inertia in the power adjustment, control price increases without bound, i.e. $\mu_{a}^{t} \rightarrow+\infty$, as $P_{a}^{t}$ approaches $X_{2}$ (from $X_{1}$ ), which is contradiction with Assumption 3 ("choking" prices).

Theorem 3.1: (Price Convergence) $\mu_{a}^{t} \rightarrow \mu_{a}^{*} \geq 0$ for all $a \in \mathcal{A}$.

Proof: Suppose the sequence $\left\{\mu_{a}^{t}: t>0\right\}$ does not converge. Therefore, either $\mu_{a}^{t} \rightarrow \infty$ or it oscillates with $\liminf \mu_{a}^{t}<\lim \sup \mu_{a}^{t}$. The former is easily discarded since large prices will result in null demand $\left(d_{m, a}^{t}=0\right.$ for all $\left.a \in \mathcal{A}\right)$ leading to $P_{a}^{t} \rightarrow 0$ which in turn implies $\mu_{a}^{t} \rightarrow 0$ which is a contradiction. Now assume that $\liminf \mu_{a}^{t}<\lim \sup \mu_{a}^{t}$, this price oscillation is only possible if the aggregate secondary power of $\operatorname{AP} a \in \mathcal{A}$, i.e. $P_{a}^{t}$, oscillates around $\bar{P}_{a}$ with $\lim \inf P_{a}^{t}<\bar{P}_{a}<\limsup P_{a}^{t}$, which contradicts Lemma 1 .

The following 3 corollaries are preliminary results to the proof of Theorem 3.2 in which we show that the limit power configuration is the efficient one.

Corollary 1 guarantees that in the limit, efficiency condition in Eq. 3.3.b is held. 
Corollary 3.1: (Complementary Slackness). $\mu_{a}^{t}\left(\bar{P}_{a}-P_{a}^{t}\right) \rightarrow 0, a \in \mathcal{A}$

Proof: If $\mu_{a}^{t} \rightarrow \mu_{a}^{*}=0$, Lemma 1 implies $\mu_{a}^{t}\left(\bar{P}_{a}-P_{a}^{t}\right) \rightarrow 0$. If $\mu_{a}^{t} \rightarrow \mu_{a}^{*}>0$ then price adjustment rule (Eq. 3.8) implies $P_{a}^{t} \rightarrow \bar{P}_{a}$.

Corollary 3.2: $\sum_{t>0} \alpha_{t}\left|\mu_{a}^{t}-\mu_{a}^{*}\right|<\infty, \quad a \in \mathcal{A}$.

Proof: See appendix.

Corollary 3.3: $\quad 0 \leq \sum_{t>0}\left(\mu_{a}^{t+1}-\mu_{a}^{t}\right)\left(P_{a}^{t+1}-\bar{P}_{a}\right)<\infty, \quad a \in \mathcal{A}$.

Proof: See appendix.

In order to give some clues on the importance of Corollary 3.3 let us describe briefly some of the findings we will cover in detail in the following subsection. In subsection 3.5.1 we study the dynamics of the joint power-profile updates by considering a function that approximates the social surplus for large values of $t$. We study the change in that approximate social surplus as the effect of power profile and control prices updates. The effect of prices update at iteration $t$ will be of the form $\left(\mu_{a}^{t+1}-\mu_{a}^{t}\right)\left(P_{a}^{t+1}-\bar{P}_{a}\right)$. Corollary 3.3 builds upon Corollary 3.2 and states that the aggregate effect of this updates throughout the auction is bounded.

\subsubsection{Power profile convergence and efficiency}

Having established that prices convergence, in this section we analyze the convergence of the joint power profile, $\mathbf{p}^{t}$, to the socially efficient configuration. Consider the function $\Psi: \mathbb{R}_{+}^{M A} \times \mathbb{R}_{+}^{A} \rightarrow \mathbb{R}$ defined as follows:

$$
\Psi(\mathbf{p}, \boldsymbol{\mu})=\sum_{m \in \mathcal{M}} U_{m}\left(r_{m}(\mathbf{p})\right)+\sum_{a \in \mathcal{A}} \mu_{a}\left(\bar{P}_{a}-\sum_{m \in \mathcal{M}} p_{m, a}\right)
$$

In light of Corollary 1 (Complementary slackness), $\Psi\left(\mathbf{p}^{t}, \boldsymbol{\mu}^{t}\right)$ is a good approximation of social surplus for large values of $t>0$.

We now prove that our algorithm is steepest ascent in the sense that power profiles are updated towards a direction close to the steepest ascent direction. Formally we have that $\left(\mathbf{d}^{t+1}-\mathbf{p}^{t}\right) \cdot \nabla_{\mathbf{p}} \Psi\left(\mathbf{p}^{t}, \boldsymbol{\mu}^{t}\right) \geq 0$. 
Proposition 3.1 (Steepest ascent property). There exist some $M>0$ such that $\left(\mathbf{d}^{t+1}-\mathbf{p}^{t}\right) \cdot \nabla_{\mathbf{p}} \Psi\left(\mathbf{p}^{t}, \boldsymbol{\mu}^{t}\right) \geq M\left\|\mathbf{d}^{t+1}-\mathbf{p}^{t}\right\|^{2} \geq 0$

Proof: It will suffice to prove that there exist some $M_{m}>0$ such that $\left(\mathbf{d}_{m}^{t+1}-\mathbf{p}_{m}^{t}\right) \cdot \nabla_{\mathbf{p}_{m}} \Psi\left(\mathbf{p}^{t}, \boldsymbol{\mu}^{t}\right) \geq M_{m}\left\|\mathbf{d}_{m}^{t+1}-\mathbf{p}_{m}^{t}\right\|^{2}$ for all $m \in \mathcal{M}$. In what follows we consider any user $m \in \mathcal{M}$ and divide the index set $\mathcal{A}$ into two sets $\mathcal{A}^{+}=\{a \in$ $\left.\mathcal{A}: d_{m, a}^{t+1}>0\right\}$ and $\mathcal{A}^{0}=\mathcal{A} \backslash \mathcal{A}^{+}$. Note that truthful update (Eq. 3.6) implies:

$$
\begin{array}{ll}
\zeta_{m, a}^{t}+\mu_{a}^{t}=U_{m}^{\prime}\left(r_{m}\left(\mathbf{d}_{m}^{t+1}, \mathbf{p}_{-m}^{t}\right)\right) \frac{\partial r_{m}}{\partial p_{m, a}}\left(\mathbf{d}_{m}^{t+1}, \mathbf{p}_{-m}^{t}\right) \geq 0 & \text { for all } a \in \mathcal{A}^{+} \\
\zeta_{m, a}^{t}+\mu_{a}^{t} \geq U_{m}^{\prime}\left(r_{m}\left(\mathbf{d}_{m}^{t+1}, \mathbf{p}_{-m}^{t}\right)\right) \frac{\partial r_{m}}{\partial p_{m, a}}\left(\mathbf{d}_{m}^{t+1}, \mathbf{p}_{-m}^{t}\right) \geq 0 & \text { for all } a \in \mathcal{A}^{0}
\end{array}
$$

Using Eq.3.11 we have:

$$
\begin{aligned}
& \left(\mathbf{d}_{m}^{t+1}-\mathbf{p}_{m}^{t}\right) \cdot \nabla_{\mathbf{p}_{m}} \Psi\left(\mathbf{p}^{t}, \boldsymbol{\mu}^{t}\right) \\
= & \sum_{a \in \mathcal{A}}\left(d_{m, a}^{t+1}-p_{m, a}^{t}\right)\left[U_{m}^{\prime}\left(r_{m}\left(\mathbf{p}^{t}\right)\right) \frac{\partial r_{m}}{\partial p_{m, a}}\left(\mathbf{p}^{t}\right)-\left(\zeta_{m, a}^{t}+\mu_{a}^{t}\right)\right] \\
= & \sum_{a \in \mathcal{A}^{+}}\left(d_{m, a}^{t+1}-p_{m, a}^{t}\right)\left[U_{m}^{\prime}\left(r_{m}\left(\mathbf{p}^{t}\right)\right) \frac{\partial r_{m}}{\partial p_{m, a}}\left(\mathbf{p}^{t}\right)-U_{m}^{\prime}\left(r_{m}\left(\mathbf{d}_{m}^{t+1}, \mathbf{p}_{-m}^{t}\right)\right) \frac{\partial r_{m}}{\partial p_{m, a}}\left(\mathbf{d}_{m}^{t+1}, \mathbf{p}_{-m}^{t}\right)\right] \\
& +\sum_{a \in \mathcal{A}^{0}}\left(-p_{m, a}^{t}\right)\left[U_{m}^{\prime}\left(r_{m}\left(\mathbf{p}^{t}\right)\right) \frac{\partial r_{m}}{\partial p_{m, a}}\left(\mathbf{p}^{t}\right)-\left(\zeta_{m, a}^{t}+\mu_{a}^{t}\right)\right] \\
\geq & \sum_{a \in \mathcal{A}}\left(d_{m, a}^{t+1}-p_{m, a}^{t}\right)\left[U_{m}^{\prime}\left(r_{m}\left(\mathbf{p}^{t}\right)\right) \frac{\partial r_{m}}{\partial p_{m, a}}\left(\mathbf{p}^{t}\right)-U_{m}^{\prime}\left(r_{m}\left(\mathbf{d}_{m}^{t+1}, \mathbf{p}_{-m}^{t}\right)\right) \frac{\partial r_{m}}{\partial p_{m, a}}\left(\mathbf{d}_{m}^{t+1}, \mathbf{p}_{-m}^{t}\right)\right] \\
= & \left(\mathbf{d}_{m}^{t+1}-\mathbf{p}_{m}^{t}\right) \cdot\left(\nabla_{\mathbf{p}_{m}} U_{m}\left(r_{m}\left(\mathbf{p}^{t}\right)\right)-\nabla_{\mathbf{p}_{m}} U_{m}\left(r_{m}\left(\mathbf{d}_{m}^{t+1}, \mathbf{p}_{-m}^{t}\right)\right)\right) \\
\geq & M_{m}\left\|\mathbf{d}_{m}^{t+1}-\mathbf{p}_{m}^{t}\right\|^{2}
\end{aligned}
$$

where the last inequality follows from Assumption 1

Note that Assumption 4 (bounded demand) altogether with power adjustment rule (Eq. 3.7) implies the existence of a compact set $\mathbb{D} \subset \mathbb{R}^{M A}$ such that $\mathbf{p}^{t}, \mathbf{d}^{t} \in \mathbb{D}$ for all $t \geq 0$. Assumption 1 and 2 implies $\nabla_{\mathbf{p}} \Psi$ is Lipschitz continuous in $\mathbb{D}$, therefore there exist some $L>0$ such that:

$$
\left|\nabla_{\mathbf{p}} \Psi(\mathbf{p}, \boldsymbol{\mu})-\nabla_{\mathbf{p}} \Psi(\tilde{\mathbf{p}}, \boldsymbol{\mu})\right| \leq L\|\mathbf{p}-\tilde{\mathbf{p}}\|
$$


for all $\mathbf{p}, \tilde{\mathbf{p}} \in \mathbb{D}, \mu \in \mathbb{R}_{+}^{A}$. This in turn implies:

$$
\left|\Psi(\tilde{\mathbf{p}}, \boldsymbol{\mu})-\Psi(\mathbf{p}, \boldsymbol{\mu})-(\tilde{\mathbf{p}}-\mathbf{p}) \cdot \nabla_{\mathbf{p}} \Psi(\mathbf{p}, \boldsymbol{\mu})\right| \leq \frac{L}{2}\|\tilde{\mathbf{p}}-\mathbf{p}\|^{2}
$$

for all $\mathbf{p}, \tilde{\mathbf{p}} \in \mathbb{D}, \mu \in \mathbb{R}_{+}^{A}$.

The following proposition uses this result and Proposition 3.1 to state the convergence of the social surplus approximation $\Psi\left(\mathbf{p}^{t}, \boldsymbol{\mu}^{t}\right)$ to some level $\tilde{\Psi} \in \mathbb{R}$.

Proposition 3.2 (Approx. Surplus Convergence). $\Psi\left(\mathbf{p}^{t}, \boldsymbol{\mu}^{t}\right) \rightarrow \tilde{\Psi} \in \mathbb{R}$

Proof: Let $\boldsymbol{P}^{t}=\left[P_{1}^{t}, \ldots, P_{A}^{t}\right]$ and $\overline{\boldsymbol{P}}=\left[\bar{P}_{1}, \ldots, \bar{P}_{A}\right]$, from Eq. 3.13 and Proposition 1, it follows that:

$$
\begin{aligned}
\Psi\left(\mathbf{p}^{t+1}, \boldsymbol{\mu}^{t+1}\right)-\Psi\left(\mathbf{p}^{t}, \mu^{t}\right)= & \Psi\left(\mathbf{p}^{t+1}, \boldsymbol{\mu}^{t+1}\right)-\Psi\left(\mathbf{p}^{t+1}, \boldsymbol{\mu}^{t}\right)+\Psi\left(\mathbf{p}^{t+1}, \boldsymbol{\mu}^{t}\right)-\Psi\left(\mathbf{p}^{t}, \boldsymbol{\mu}^{t}\right) \\
\geq & \left(\boldsymbol{\mu}^{t+1}-\boldsymbol{\mu}^{t}\right) \cdot\left(\overline{\boldsymbol{P}}-\boldsymbol{P}^{t+1}\right)+\left(\mathbf{p}^{t+1}-\mathbf{p}^{t}\right) \cdot \nabla_{\mathbf{p}} \Psi\left(\mathbf{p}^{t}, \boldsymbol{\mu}^{t}\right) \\
& -\frac{L}{2}\left\|\mathbf{p}^{t+1}-\mathbf{p}^{t}\right\|^{2} \\
\geq & \left(\boldsymbol{\mu}^{t+1}-\boldsymbol{\mu}^{t}\right) \cdot\left(\overline{\boldsymbol{P}}-\boldsymbol{P}^{t+1}\right)+\alpha_{t}\left(\mathbf{d}^{t+1}-\mathbf{p}^{t}\right) \cdot \nabla_{\mathbf{p}} \Psi\left(\mathbf{p}^{t}, \boldsymbol{\mu}^{t}\right) \\
& -\frac{L}{2} \alpha_{t}^{2}\left\|\mathbf{d}^{t+1}-\mathbf{p}^{t}\right\|^{2} \\
= & -\left(\boldsymbol{\mu}^{t+1}-\boldsymbol{\mu}^{t}\right) \cdot\left(\boldsymbol{P}^{t+1}-\overline{\boldsymbol{P}}\right)-\frac{L \bar{d}^{2}}{2} \alpha_{t}^{2}
\end{aligned}
$$

where $\bar{d}>0$ is the bound on the norm of joint demand and power profiles (see Assumption 4). From Corollary 3.3 it follows that $\Psi\left(\mathbf{p}^{t}, \boldsymbol{\mu}^{t}\right)$ does not go to $-\infty$ neither it oscillates. Thus boundedness (above) of $\Psi\left(\mathbf{p}^{t}, \boldsymbol{\mu}^{t}\right)$ implies convergence.

Proposition 3.3 (Limit Stationarity). $\left\|\mathbf{d}^{t+1}-\mathbf{p}^{t}\right\| \rightarrow 0$

Proof: Similar as in the proof of Proposition 2 we have

$$
\begin{aligned}
\Psi\left(\mathbf{p}^{t+1}, \boldsymbol{\mu}^{t+1}\right)-\Psi\left(\mathbf{p}^{t}, \mu^{t}\right) \geq & \left(\boldsymbol{\mu}^{t+1}-\boldsymbol{\mu}^{t}\right) \cdot\left(\overline{\boldsymbol{P}}-\boldsymbol{P}^{t+1}\right)+\alpha_{t}\left(\mathbf{d}^{t+1}-\mathbf{p}^{t}\right) \cdot \nabla_{\mathbf{p}} \Psi\left(\mathbf{p}^{t}, \boldsymbol{\mu}^{t}\right) \\
& -\alpha_{t}^{2} \frac{L \bar{d}^{2}}{2}
\end{aligned}
$$


where again $\bar{d}>0$ is the bound on the norm of joint demand and power profiles (see Assumption 4). From proposition 3.2 and Corollary 3.3 it follows that

$$
\begin{aligned}
\sum_{t>0} \alpha_{t}\left(\mathbf{d}^{t+1}-\mathbf{p}^{t}\right) \cdot \nabla_{\mathbf{p}} \Psi\left(\mathbf{p}^{t}, \boldsymbol{\mu}^{t}\right) \leq & \tilde{\Psi}-\Psi\left(\mathbf{p}^{0}, \boldsymbol{\mu}^{0}\right)-\sum_{t>0}\left(\boldsymbol{\mu}^{t+1}-\boldsymbol{\mu}^{t}\right)\left(\overline{\boldsymbol{P}}-\boldsymbol{P}^{t+1}\right) \\
& +\sum_{t>0} \frac{L \bar{d}^{2}}{2} \alpha_{t}^{2} \\
< & \infty
\end{aligned}
$$

Hence, $\left(\mathbf{d}^{t+1}-\mathbf{p}^{t}\right) \cdot \nabla_{\mathbf{p}} \Psi\left(\mathbf{p}^{t}, \boldsymbol{\mu}^{t}\right) \rightarrow 0$. Which in light of Theorem 3.1 (price convergence) becomes $\left(\mathbf{d}^{t+1}-\mathbf{p}^{t}\right) \cdot \nabla_{\mathbf{p}} \Psi\left(\mathbf{p}^{t}, \boldsymbol{\mu}^{*}\right) \rightarrow 0$. Finally Proposition 3.1 gives us $\left\|\mathbf{d}^{t+1}-\mathbf{p}^{t}\right\| \rightarrow 0$.

Theorem 3.2. $\mathbf{p}^{t} \rightarrow \mathbf{p}^{*} \in \mathbb{R}_{+}^{M A}$ where $\boldsymbol{p}^{*}$ is the unique solution of problem in (Eq. 3.2).

Proof: Note that limit stationarity in addition to truthful demand (see Eq.3.12) implies that for $t \rightarrow \infty$ :

$$
U_{m}^{\prime}\left(r_{m}\left(\mathbf{p}^{t}\right)\right) \frac{\partial r_{m}}{\partial p_{m, a}}\left(\mathbf{p}^{t}\right)-\left(\mu_{a}^{*}+\zeta_{m, a}^{t}\right) \leq 0 \quad \text { for all } m \in \mathcal{M}, a \in \mathcal{A}
$$

with equality if $p_{m, a}^{t}=0$ which resembles efficiency condition in (Eq. 3.3.a). This altogether with Corollary 3.1 implies that any limit configuration is efficient, furthermore, strict concavity of the social surplus (Assumption 2) implies uniqueness of the efficient configuration. Hence $\mathbf{p}^{t} \rightarrow \mathbf{p}^{*}$ where $\mathbf{p}^{*}$ is the unique solution of problem in (Eq. 3.2).

\subsection{Incentive Compatibility}

Convergence and Efficiency results (Theorems 3.1 and 3.2) relay on the assumption that users are truthful, that is, at each iteration $t>0$ each user $m \in \mathcal{M}$ updates his power profile as stated in Equations (3.6) and (3.7) and truthfully 
reports $U_{m}^{\prime}\left(\mathbf{p}^{t}\right)$. In this section we prove that it is in the best interest each user to be truthful no matter the behavior of his competitors.

First note that the auctioneer can impose an activity rule stating that power profiles of user $m \in \mathcal{M}$ must be consistent with some strongly concave utility function $\widetilde{U}_{k}() \in \mathbb{U}$ where $\mathbb{U}$ is the set of strongly concave functions from $\mathbb{R}_{+}$to $\mathbb{R}$. That is, $k$ 's power profile must be updated as follows:

$$
\begin{gathered}
\mathbf{p}_{m}^{t}=\mathbf{p}_{m}^{t-1}+\alpha_{t}\left(\widetilde{\mathbf{d}}_{m}^{t}-\mathbf{p}_{m}^{t-1}\right) \quad \text { where } \\
\widetilde{\mathbf{d}}_{m}^{t}=\arg \max _{\mathbf{d}_{m} \in \mathbb{R}_{+}^{A}}\left[\widetilde{U}_{m}\left(r_{m}\left(\mathbf{d}_{m}, \mathbf{p}_{-m}^{t-1}\right)\right)-\mathbf{d}_{m} \cdot\left(\boldsymbol{\mu}^{t-1}+\boldsymbol{\zeta}_{m}^{t-1}\right)\right]
\end{gathered}
$$

for some function $\widetilde{U}_{m} \in \mathbb{U}$.

Note that at time $t>0$ user $m \in \mathcal{M}$ directly reports $U_{m}^{\prime}\left(r_{m}\left(\mathbf{p}^{t}\right)\right)$ and indirectly (through the profile update) reports $U_{m}^{\prime}\left(r_{m}\left(\mathbf{d}^{t}, \mathbf{p}^{t-1}\right)\right)$. The auctioneer can then enforce the activity rule above by checking that $U_{m}^{\prime}()$ is strictly decreasing for all $m \in \mathcal{M}$. Also since $\mu_{a}^{0}=\bar{\mu}$ for all $a \in \mathcal{A}$ it is reasonable to impose an additional activity rule stating that $\mathbf{d}_{m}^{1}=\mathbf{0} \in \mathbb{R}_{+}^{A}$. Any user violating this activity rules will be penalized with a large payment and removed from the auction. This penalization will guarantee that all users will update in a way that is consistent with some utility function from the space $\mathbb{U}$.

In what follows we shall consider a strategy by user $m \in \mathcal{M}$ in which he behaves as if his utility function were $\tilde{U}_{m} \in \mathbb{U}$. To keep notation consistent we use $\widetilde{d}_{m, a}^{t}\left(\right.$ instead of $\left.d_{m, a}^{t}\right)$ to denote power demand of user $m \in \mathcal{M}$ at AP $a \in \mathcal{A}$ and $\widetilde{\tau}_{m}^{t}$ (instead of $\tau_{m}^{t}$ ) to denote the cumulative payment of user $m \in \mathcal{M}$ at time $t>0$. The following Lemma describes the resulting payments to be assessed on users. The characterization of these payments will be used later to show that it is an $\epsilon$-dominant strategy to update power profile truthfully (i.e. according to Equations 3.6 and 3.7).

Lemma 3.2: When users employ utility functions $\left\{\widetilde{U}_{m} \in \mathbb{U}\right\}_{m \in \mathcal{M}}$, to calculate demands as in Eq.(3.14) then power profiles and prices converge (i.e. $\widetilde{\mathbf{p}}^{t} \rightarrow \widetilde{\mathbf{p}} \in$ 
$\left.R_{+}^{M A}, \tilde{\boldsymbol{\mu}}^{t} \rightarrow \widetilde{\boldsymbol{\mu}} \in R_{+}^{A}\right)$ and

$$
\lim _{t \rightarrow \infty} \widetilde{\tau_{m}^{t}}=\sum_{i \in \mathcal{M} \backslash\{m\}} \widetilde{U}_{i}(0)-\sum_{i \in \mathcal{M} \backslash\{m\}} \widetilde{U}_{i}\left(r_{i}(\widetilde{\mathbf{p}})\right)+\tilde{\delta}_{m}(\rho)
$$

where $\widetilde{\delta}_{m}(\rho): \mathbb{R}_{+} \mapsto \mathbb{R}$ is a mapping such that $\left|\widetilde{\delta}_{m}(\rho)\right| \rightarrow 0^{+}$as $\rho \rightarrow 0^{+}$.

\section{Proof:}

The formal proof is given in the appendix. Here we provide a sketch. Prices and joint power profile convergence are immediate by Theorems 3.1 and 3.2 when users behave as dictated by intrinsic utility functions (or strategies) $\left\{\widetilde{U}_{m}\right\}_{m \in \mathcal{M}}$.

The payment rule defined in Eq.(3.10) can be interpreted as if at time $t>0$, user $m \in \mathcal{M}$ were charged $-\sum_{i \neq m}\left[r_{i}\left(\mathbf{p}^{t}\right)-r_{i}\left(\mathbf{p}^{t-1}\right)\right] \widetilde{U}_{i}^{\prime}\left(r_{i}\left(\mathbf{p}^{t}\right)\right)$ which approximates the negative change in utility of his competitors from iteration $t-1$ to iteration $t$. Hence in the limit, user $m \in \mathcal{M}$ is assessed a total payment that approximates the change in aggregate utility of all other users (as measured by $\left\{\tilde{U}_{i}: i \neq m\right\}$ ). The term $\widetilde{\delta}_{m}(\rho)$ is an approximation error that emerges as a result of having discrete changes in prices and power profiles. Since an infinitesimal $\rho>0$ yields infinitesimal changes in prices and power profiles, the approximation error is determined by the magnitude of $\rho>0$.

Note that setting payments to the negative utility of his competitors will certainly help each user $m \in \mathcal{M}$ internalize the effect he may have in the social surplus. As proved in the following theorem, this will set the incentives to behave truthfully.

At this point we are interested in the overall outcome (joint power profile and payments) of the auction. Note that the final (joint) power profile will be the efficient one for the active users (i.e. those that have not been removed from the auction) independently of the the initial one. Note from Lemma 3.2 that payments are designed to align each user incentives with those of the auctioneer (maximize the social surplus). Since the social planner does not care about utility of users removed from the auction, these users should not be considered in the payment of 
the active ones. Hence a small modification in the payment rule is required. Let $\widetilde{\mathcal{M}}$ be the set of users that followed the activity rules until the final iteration, say $T>0$. The cumulative payment of user $m \in \widetilde{\mathcal{M}}$ after iteration $t>0, \tau_{m}^{t}$ should be redefined as follows

$$
\tau_{m}^{t}=\tau_{m}^{t-1}-\sum_{i \in \widehat{\mathcal{M}} \backslash\{m\}}\left[r_{i}\left(\mathbf{p}^{t}\right)-r_{i}\left(\mathbf{p}^{t-1}\right)\right] U_{i}^{\prime}\left(r_{i}\left(\mathbf{p}^{t}\right)\right)
$$

for all $t \geq 1$ and $\tau_{m}^{0}=0$. Note that we are using the same rule stated initially (see Eq.(3.10)) but considering only those users in $\widetilde{\mathcal{M}}$. Following a similar analysis as in Lemma 3.2 it follows that payment of user $m \in \widetilde{\mathcal{M}}$ is:

$$
\lim _{t \rightarrow \infty} \widetilde{\tau_{m}^{t}}=\sum_{i \in \widetilde{\mathcal{M}} \backslash\{m\}} \widetilde{U}_{i}(0)-\sum_{i \in \widetilde{\mathcal{M}} \backslash\{m\}} \widetilde{U}_{i}\left(r_{i}(\widetilde{\mathbf{p}})\right)+\tilde{\delta}_{m}(\rho)
$$

Note that efficiency and the characterization of payments in Lemma 3.2 hold as if only users $\widetilde{\mathcal{M}} \subseteq \mathcal{M}$ had participated in the auction.

Theorem 3.3: Being truthful is an $\epsilon$-Dominant Strategy in the sense that for any user, the maximum benefit he can attain by not being truthful is $\epsilon(\rho)$ where $\epsilon(\rho) \rightarrow 0$ as $\rho \rightarrow 0^{+}$.

Proof: Let $\widetilde{U}_{i} \in \mathbb{U}$ be the strongly concave function that characterizes behavior of user $i \in \widetilde{\mathcal{M}}$. To avoid unnecessary notation, in what follows $M$ should be interpreted as the cardinality of $\widetilde{\mathcal{M}}$. By arguments similar to those in Theorems 3.1 and 3.2 it follows that the limiting allocation, say $\widetilde{\mathbf{p}} \in \mathbb{R}_{+}^{M A}$ is the solution to the optimization problem:

$$
\begin{array}{ll}
\max _{\mathbf{p} \in \mathbb{R}_{+}^{M A}} & \sum_{i \in \widetilde{\mathcal{M}}} \widetilde{U}_{i}\left(r_{i}(\mathbf{p})\right) \\
\text { s.t. } & \\
& \sum_{i \in \widetilde{\mathcal{M}}} p_{i, a} \leq \bar{P}_{a} \quad a \in \mathcal{A}
\end{array}
$$


Using Lemma 3.2, the overall payment of user $m \in \widetilde{\mathcal{M}}$ is

$$
\lim _{t \rightarrow \infty} \widetilde{\tau_{m}^{t}}=\sum_{i \in \mathcal{M} \backslash\{m\}} \widetilde{U}_{i}(0)-\widetilde{\delta}_{m}(\rho)-\sum_{i \in \mathcal{M} \backslash\{m\}} \widetilde{U}_{i}\left(r_{i}(\widetilde{\mathbf{p}})\right)
$$

Hence the overall profit of user $m \in \mathcal{M}$ is:

$$
\begin{aligned}
\widetilde{V}_{m} & =U_{m}\left(r_{m}(\widetilde{\mathbf{p}})\right)-\lim _{t \rightarrow \infty} \widetilde{\tau_{m}^{t}} \\
& =U_{m}\left(r_{m}(\widetilde{\mathbf{p}})\right)+\sum_{i \in \mathcal{M} \backslash\{m\}} \widetilde{U}_{i}\left(r_{i}(\widetilde{\mathbf{p}})\right)-\sum_{i \in \mathcal{M} \backslash\{m\}} \widetilde{U}_{i}(0)-\widetilde{\delta}_{m}(\rho)
\end{aligned}
$$

Now assume $m$ reports and updates truthfully (i.e. using $U_{m}$ instead of $\widetilde{U}_{m}$ ) while all other users $i \in \widetilde{M} \backslash\{m\}$ report and update according to some $\widetilde{U}_{i} \in \mathbb{U}$. Abusing notation, let us denote by $\widehat{\mathbf{p}} \in \mathbb{R}_{+}^{M A}$, the limit joint power profile. Again, by Theorems 3.1 and 3.2 it follows that $\widehat{\mathbf{p}}$ is the solution to

$$
\max _{\mathbf{p} \in \mathbb{R}_{+}^{M A}} U_{m}\left(r_{m}(\mathbf{p})\right)+\sum_{i \in \widetilde{\mathcal{M}} \backslash\{m\}} \widetilde{U}_{i}\left(r_{i}(\mathbf{p})\right)
$$

s.t.

$$
\sum_{i \in \widehat{\mathcal{M}} \backslash\{m\}} p_{i, a} \leq \bar{P}_{a} \quad a \in \mathcal{A}
$$

and the overall profit of $m$ is:

$$
\begin{aligned}
V_{m}^{*} & =U_{m}\left(r_{m}(\widehat{\mathbf{p}})\right)-\lim _{t \rightarrow \infty} \widehat{\tau_{m}^{t}} \\
& =U_{m}\left(r_{m}(\widehat{\mathbf{p}})\right)+\sum_{i \in \mathcal{M} \backslash\{m\}} \widetilde{U}_{m}\left(r_{m}(\widehat{\mathbf{p}})\right)-\sum_{i \in \mathcal{M} \backslash\{m\}} \widetilde{U}_{i}(0)-\widehat{\delta}_{m}(\rho)
\end{aligned}
$$

By optimality of $\widehat{\mathbf{p}}$ we have

$$
\widetilde{V}_{m}-V_{m}^{*} \leq-\widetilde{\delta}_{m}(\rho)+\widehat{\delta}_{m}(\rho) \leq\left|\widetilde{\delta}_{m}(\rho)\right|+\left|\widehat{\delta}_{m}(\rho)\right|
$$

Finally, by selecting $\rho$ small enough we can guarantee $\left|\widetilde{\delta}_{m}(\rho)\right|+\left|\widehat{\delta}_{m}(\rho)\right|$ is as small as desired.

We have proved that the maximum benefit user $m \in \mathcal{M}$ can attain by using some function $\widetilde{U}_{m} \in \mathbb{U}$ such that $\widetilde{U}_{m} \neq U_{m}$ goes to zero as $\rho \rightarrow 0^{+}$. Using some 
strategy that can not be described by some function $\widetilde{U}_{m} \in \mathbb{U}$ will be revealed by checking the activity rule, in which case a large penalty will be charged, hence being truthful is an $\epsilon$-Dominant strategy in the sense that for any user, the maximum benefit he can attain by not being truthful is $\epsilon(\rho)$ where $\epsilon(\rho) \rightarrow 0$ as $\rho \rightarrow 0^{+}$

\subsection{Conclusion}

This chapter introduces Inertial Temperature Auction (ITA), a dynamic auction design that allows a primary network provide uplink access to secondary users, subject to restrictions in the service quality of the primary users. We account for several access points, with heterogeneous (residual) capacity or temperature. ITA achieves power control (in order to guarantee quality of the primary users) by means of control prices, one per access point; these prices are updated as a function of the excessive power from secondary users. ITA also uses a discriminative externality price that allows secondary users internalize the effect they have in the social surplus. These prices guide secondary users towards the efficient configuration. A very distinctive characteristic of the Inertial Temperature Auction is the inertia in the power profile updates, which provides the means for coordination and eliminates cycles and erratic behaviors observed when using other designs that do not consider externalities. More importantly all of the features mentioned are achieved in $\epsilon$-dominant strategies, that is, each rational user has the incentives to behave as requested by the algorithm, independently of the behavior of his competitors. 


\subsection{Appendix of Inertial Temperature Auction}

\subsubsection{Proof of concavity}

In this section we study the problem of two secondary users that want to establish an uplink connection with an access point. These users have utility functions $U_{m}\left(r_{m}\right)=q r_{m}$ where $m \in\{1,2\}$. Assume system gain is $G$, noise power is $N$, and power from primary users is $I$. We prove that a sufficient condition for concavity of the objective function $O F\left(p_{1}, p_{2}\right)=\sum_{m \in\{1,2\}} U_{m}\left(r_{m}\left(p_{1}, p_{2}\right)\right)$, were $p_{m}$ is the power received from secondary user $m \in\{1,2\}$ at the AP, is as follows:

$$
\frac{1 / \sqrt{2}}{N+T+G(I+T)}>\frac{\sqrt{2 G}}{N+G I}
$$

Note that we have omitted the AP subscripts since we only have one. It can be proved that

$$
\begin{aligned}
\frac{\partial^{2} O F\left(p_{1}, p_{2}\right)}{\partial p_{1} \partial p_{2}}= & -\frac{q G}{\ln (2)}\left[\frac{1}{\left(N+G\left(I+p_{1}\right)+p_{2}\right)^{2}}+\frac{1}{\left(N+G\left(I+p_{2}\right)+p_{1}\right)^{2}}\right] \\
\frac{\partial^{2} O F\left(p_{1}, p_{2}\right)}{\partial p_{m} \partial p_{m}}= & \frac{q}{\ln (2)}\left[\frac{G^{2}}{\left(N+G\left(I+p_{m}\right)\right)^{2}}-\frac{G^{2}}{\left(N+G\left(I+p_{m}\right)+p_{j}\right)^{2}}\right. \\
& \left.-\frac{1}{\left(N+G\left(I+p_{j}\right)+p_{m}\right)^{2}}\right]
\end{aligned}
$$

for $m \in\{1,2\}$ and $j \neq m$. Since $G<1$ and $p_{m} \leq T$ for $m \in\{1,2\}$ we have

$$
\begin{aligned}
\frac{1 / 2}{\left(N+G\left(I+p_{j}\right)+p_{m}\right)^{2}} & \geq \frac{1 / 2}{(N+G(I+T)+T)^{2}} \\
& >\frac{2 G}{(N+G I)^{2}} \\
& >\frac{G^{2}}{(N+G I)^{2}} \\
& \geq \frac{G^{2}}{\left(N+G\left(I+p_{m}\right)\right)^{2}}
\end{aligned}
$$


It follows that $\frac{\partial^{2} O F\left(p_{1}, p_{2}\right)}{\partial p_{m} \partial p_{m}}<0$ for $m \in\{1,2\}$. Furthermore $\left|\frac{\partial^{2} O F\left(p_{1}, p_{2}\right)}{\partial p_{m} \partial p_{m}}\right|>$ $\frac{q / 2}{\ln (2)(N+G(I+T)+T)^{2}}$, hence

$$
\begin{aligned}
\left|\frac{\partial^{2} O F\left(p_{1}, p_{2}\right)}{\partial p_{1} \partial p_{2}}\right| & =\frac{q G}{\ln (2)}\left[\frac{1}{\left(N+G\left(I+p_{1}\right)+p_{2}\right)^{2}}+\frac{1}{\left(N+G\left(I+p_{2}\right)+p_{1}\right)^{2}}\right] \\
& \leq \frac{q}{\ln (2)}\left[\frac{2 G}{(N+G I)^{2}}\right] \\
& <\frac{q}{\ln (2)} \frac{1 / 2}{(N+T+G(I+T))^{2}} \\
& <\left|\frac{\partial^{2} O F\left(p_{1}, p_{2}\right)}{\partial p_{m} \partial p_{m}}\right|
\end{aligned}
$$

It follows that matrix of the objective function is diagonally dominant and negative definite, hence concavity of the objective function.

\subsubsection{Proof of Lemma 3.1}

Suppose $\lim \sup P_{a}^{t}>\bar{P}_{a}$. So either $P_{a}^{t}$ grows unboundedly (i.e. $P_{a}^{t} \rightarrow \infty$ ) or it converges to a level greater than temperature threshold $\bar{P}_{a}$ (i.e. $P_{a}^{t} \rightarrow P_{a}^{*}>\bar{P}_{a}$ ) or it oscillates with $\lim \sup P_{a}^{t}>\bar{P}_{a}$. The first two options are easily discarded since both imply $\mu_{a}^{t} \rightarrow \infty$, hence Assumption 3 implies $P_{a}^{t} \rightarrow 0$, a contradiction. Therefore we focus on discarding the latter option of oscillating $P_{a}^{t}$ with $\limsup P_{a}^{t}>\bar{P}_{a}$, by contradiction:

1. Assume that $P_{a}^{t}$ oscillates with $\lim \sup P_{a}^{t}>\bar{P}_{a}$. Therefore there exist some secondary power levels $X_{1}, X_{2}$ on AP $a \in \mathcal{A}$ with $\bar{P}_{a}<X_{1}<X_{2}<\limsup P_{a}^{t}$ such that $X_{1}$ and $X_{2}$ are up-crossed and down-crossed infinitely often.

2. Let $T$ be an up-crossing of $X_{2}$ and $Y(T)$ be the last up-crossing of $X_{1}$ before $T$. That is, $T$ is some $t>0$ such that $P_{a}^{T-1}<X_{2} \leq P_{a}^{T}$ and $Y(T)=\sup _{t \leq T}\left\{P_{a}^{t-1}<X_{1} \leq P_{a}^{t}\right\}$. Note that for any $T$ and $t \in[Y(T), T-1]$ we have:

- $X_{2}>P_{a}^{t} \geq X_{1}>\bar{P}_{a}$, therefore

- $\mu_{a}^{t+1}-\mu_{a}^{t}=\rho\left(P_{a}^{t}-\bar{P}_{a}\right)$ 
3. Now we prove that the time it takes to go from $Y(T)$ to $T$ is (unboundedly) increasing in $\mathrm{T}$. This implies that the price increase from $Y(T)$ to $T$ also grows (unboundedly) with $T$. Formally we have:

$$
\begin{aligned}
X_{2}-X_{1} & \leq P_{a}^{T}-P_{a}^{Y(T)-1} \\
& \leq \sum_{t=Y(T)}^{T}\left\|P_{a}^{t}-P_{a}^{t-1}\right\| \\
& \leq \sum_{t=Y(T)}^{T} \alpha_{t} \bar{d} \\
& \leq(\Delta T+1) \alpha_{Y(T)} \bar{d}
\end{aligned}
$$

where $\Delta T=T-Y(T)$ and $\bar{d}$ is as defined in Assumption 4. The former implies $\frac{X_{2}-X_{1}}{\alpha_{Y(T)}}<\bar{d}(\Delta T+1)$. Which in turn implies $\Delta T \rightarrow \infty$ as $T \rightarrow \infty$ (the time to go from $X_{1}$ to $X_{2}$ increases unboundedly with $T$ ).

Now notice that:

$$
\begin{aligned}
\mu_{a}^{T-1} & =\mu_{a}^{Y(T)}+\sum_{t=Y(T)}^{T-2}\left(\mu_{a}^{t+1}-\mu_{a}^{t}\right) \\
& =\mu_{a}^{Y(T)}+\sum_{t=Y(T)}^{T-2} \rho\left(P_{a}^{t}-\bar{P}_{a}\right) \\
& \geq \mu_{a}^{Y(T)}+\sum_{t=Y(T)}^{T-2} \rho\left(X_{1}-\bar{P}_{a}\right) \\
& \geq \mu_{a}^{Y(T)}+\Delta T \rho\left(X_{1}-\bar{P}_{a}\right)
\end{aligned}
$$

where the second and third lines come from the properties itemized on 2). Therefore the price at $T-1$ increases (unboundedly) with T. i.e. $\mu_{a}^{T-1} \rightarrow \infty$ as $T \rightarrow \infty$.

4. Suppose we select an up-crossing $T \rightarrow \infty$ therefore $\mu_{a}^{T-1} \rightarrow \infty$, assumption 3 (choking prices) implies $d_{m, a}^{T}=0$ for all $m \in \mathcal{M}$ which in turn implies $P_{a}^{T}=P_{a}^{T-1}\left(1-\alpha_{T}\right)<P_{a}^{T-1}$. This contradicts the hypothesis that $T$ is an up-crossing of $X_{2}$ (i.e. that $P_{a}^{T-1}<X_{2}<P_{a}^{T}$ ). 


\subsubsection{Proof of Corollary 3.2}

Let us write the price update as follows

$$
\mu_{a}^{t+1}=\mu_{a}^{t}+\rho_{t}\left(P_{a}^{t+1}-\bar{P}_{a}\right)
$$

where

$$
\rho_{t}= \begin{cases}\rho & \mu_{a}^{t}+\rho\left(P_{a}^{t+1}-\bar{P}_{a}\right)>0 \\ \frac{\mu_{a}^{t}}{\bar{P}_{a}-P_{a}^{t+1}} & \mu_{a}^{t}+\rho\left(P_{a}^{t+1}-\bar{P}_{a}\right) \leq 0\end{cases}
$$

Now let $T(t)=\inf \left\{T \geq t|| \mu_{e}^{\tau}-\mu_{e}^{*} \mid<\frac{1}{t}\right.$ for all $\left.\tau \geq T\right\}$. We can write

$$
\begin{aligned}
\left|\mu_{a}^{t}-\mu_{a}^{*}\right| & =\left|\mu_{a}^{*}-\mu_{a}^{T(t)}+\mu_{a}^{T(t)}-\mu_{a}^{t}\right| \\
& =\left|\mu_{a}^{*}-\mu_{a}^{T(t)}+\sum_{\ell=t}^{T(t)-1} \rho_{t}\left(P_{a}^{\ell+1}-\bar{P}_{a}\right)\right| \\
& =\frac{1}{\alpha_{t}}\left|\alpha_{t}\left(\mu_{a}^{*}-\mu_{a}^{T(t)}\right)+\alpha_{t} \sum_{\ell=t}^{T(t)-1} \rho_{t}\left(P_{a}^{\ell+1}-\bar{P}_{a}\right)\right|
\end{aligned}
$$

Since $\left|\mu_{a}^{t}-\mu_{a}^{*}\right| \rightarrow 0$ it follows that there exists some $L>0$ and $\gamma>1$ such that

$$
\left|\alpha_{t}\left(\mu_{a}^{*}-\mu_{a}^{T(t)}\right)+\alpha_{t} \sum_{\ell=t}^{T(t)-1} \rho_{t}\left(P_{a}^{\ell+1}-\bar{P}_{a}\right)\right| \leq L\left(\alpha_{t}\right)^{\gamma}
$$

Thus

$$
\begin{aligned}
\sum_{t>0} \alpha_{t}\left|\mu_{a}^{t}-\mu_{a}^{*}\right| & \leq \sum_{t>0}\left|\mu_{a}^{*}-\mu_{a}^{T(t)}+\sum_{\ell=t}^{T(t)-1} \rho_{t}\left(P_{a}^{\ell+1}-\bar{P}_{a}\right)\right| \\
& \leq \sum_{t>0} L\left(\alpha_{t}\right)^{\gamma}<\infty
\end{aligned}
$$

\subsubsection{Proof of Corollary 3.3}

Note from the price update rule (Eq. 3.8) that if $P_{a}^{t+1}-\bar{P}_{a}>0$ then $\mu_{a}^{t+1}-\mu_{a}^{t}>$ 0 , similarly if $P_{a}^{t+1}-\bar{P}_{a} \leq 0$ then $\mu_{a}^{t+1}-\mu_{a}^{t} \leq 0$. Hence, $\left(\mu_{a}^{t+1}-\mu_{a}^{t}\right)\left(P_{a}^{t+1}-\bar{P}_{a}\right) \geq 0$ 
for all $t>0$. By Abel's Lemma and Corollaries 1 and 2 we have:

$$
\begin{aligned}
\sum_{t>0}\left(\mu_{a}^{t+1}-\mu_{a}^{t}\right)\left(P_{a}^{t+1}-\bar{P}_{a}\right) & =\sum_{t>0}\left[\left(\mu_{a}^{t+1}-\mu_{a}^{*}\right)-\left(\mu_{a}^{t}-\mu_{a}^{*}\right)\right]\left(P_{a}^{t+1}-\bar{P}_{a}\right) \\
& =\lim _{t \rightarrow \infty}\left[\left(\mu_{a}^{t}-\mu_{a}^{*}\right)\left(P_{a}^{t+1}-\bar{P}_{a}\right)\right]-\sum_{t>0}\left(\mu_{a}^{t}-\mu_{a}^{*}\right)\left(P_{a}^{t+1}-P_{a}^{t}\right) \\
& =\sum_{t>0}\left(\mu_{a}^{t}-\mu_{a}^{*}\right)\left(P_{a}^{t}-P_{a}^{t+1}\right) \\
& \leq \bar{d} \sum_{t>0} \alpha_{t}\left|\mu_{a}^{t}-\mu_{a}^{*}\right|<\infty
\end{aligned}
$$

\subsubsection{Proof of Lemma 3.2}

Although this proof has the same intuition that the one in Section 2.6 the way we proceed here is simpler. Note that we have used a high level payment rule which implementation has already been discussed, this will certainly help us provide a very simple and descriptive proof.

To keep notation consistent, we use $\widetilde{\boldsymbol{p}}^{t}$ (instead of $\boldsymbol{p}^{t}$ ) to denote the power profile at time $t \in\{0,1, \ldots\}$. We will need a continuous time approximation of the discrete time power profile. For this let $\widetilde{\boldsymbol{p}}(t)$ be a linear interpolation of $\widetilde{\boldsymbol{p}}^{t}$ (i.e. $\widetilde{\boldsymbol{p}}(t)=\widetilde{\boldsymbol{p}}^{\lfloor t\rfloor}+\frac{t-\lfloor t\rfloor}{\lceil t\rceil-\lfloor t\rfloor}\left(\widetilde{\boldsymbol{p}}^{\lceil t\rceil}-\widetilde{\boldsymbol{p}}^{\lfloor t\rfloor}\right)$ where $\lfloor x\rfloor$ and $\lceil x\rceil$ represent the floor and ceiling functions respectively).

After $T>0$ rounds, it holds that

$$
\widetilde{\tau}_{m}^{T}=-\sum_{t=0}^{T-1} \sum_{i \neq m}\left[r_{i}\left(\widetilde{\mathbf{p}}^{t+1}\right)-r_{i}\left(\widetilde{\mathbf{p}}^{t}\right)\right] U_{i}^{\prime}\left(r_{i}\left(\widetilde{\mathbf{p}}^{t+1}\right)\right)
$$

for all $m \in \mathcal{M}$. By convergence, we can approximate $\widetilde{\tau}_{m}^{T}$ with Riemann-Stieltjes integral in the following sense:

$$
\widetilde{\tau}_{m}^{T}=-\sum_{i \neq m_{t=1}} \int_{i}^{T} U_{i}^{\prime}\left(r_{i}(\widetilde{\mathbf{p}}(t))\right) \partial r_{i}(\widetilde{\mathbf{p}}(t))+\widetilde{\epsilon}_{m}^{T}(\rho)
$$

where the term $\widetilde{\epsilon}_{m}^{T}(\rho)$ accounts for the error induced by the approximation. Note however that as $\rho \rightarrow 0$, changes in prices and demands become infinitesimal, improving the approximation such that $\tilde{\epsilon}_{m}^{T}(\rho) \rightarrow 0$ as $\rho \rightarrow 0$ for all $T>0$. 
Using the gradient theorem we have that:

$$
\widetilde{\tau}_{m}^{T}=-\sum_{i \neq m}\left[U_{i}\left(r_{i}\left(\widetilde{\mathbf{p}}^{T}\right)\right)-U_{i}\left(r_{i}\left(\widetilde{\mathbf{p}}^{1}\right)\right)\right]+\widetilde{\epsilon}_{m}^{T}(\rho)
$$

Finally, since $\widetilde{\mathbf{p}}^{T} \rightarrow \widetilde{\mathbf{p}}$ and $\widetilde{\mathbf{p}}^{1}=\mathbf{0}$

$$
\lim _{T \rightarrow \infty} \widetilde{\tau}_{m}^{T}=-\sum_{i \neq m}\left[U_{i}\left(r_{i}(\widetilde{\mathbf{p}})\right)-U_{i}(0)\right]+\widetilde{\delta}_{m}(\rho)
$$

where

$$
\delta_{m}(\rho)=\lim _{T \rightarrow \infty} \epsilon_{m}^{T}(\rho)
$$

which finalizes the proof of this Lemma. 


\section{Chapter 4}

\section{Truthful Multichannel Auction}

\subsection{Introduction}

Increasing demand for mobile broadband applications has created the need for new dynamic and efficient methods that reallocate the available spectrum in the short run. In light of this need, the FCC is currently working on laying the groundwork for voluntary "incentive auctions" that would provide the means for efficient spectrum re-allocation in the short run (see www.fcc.gov/topic/incentiveauctions). Auction-based mechanisms have been successfully used to allocate licenses for long-term commercial exploitation of the spectrum (see [10]). In this long term context, auction designs had to take into account inherent complementarities or synergies that could exist across licenses over adjacent geographic areas. However, the challenges posed by short term re-allocation are significantly different. For example, when the spectrum is partitioned into channels, each of these channels can be allocated to several networks when the potential for cross interference among them is minimal. This interference can be controlled by imposing constraints on the allocations so that total interference is kept between given acceptable levels. To illustrate this, consider the situation depicted in Figure 4.1a associated with the use of a single channel. Here, there is one large network (Network 2) operating contiguously with two small networks (Networks 1 and 3). Given the geographical layout of the networks, if the channel is allocated to network 2, then it can not be 


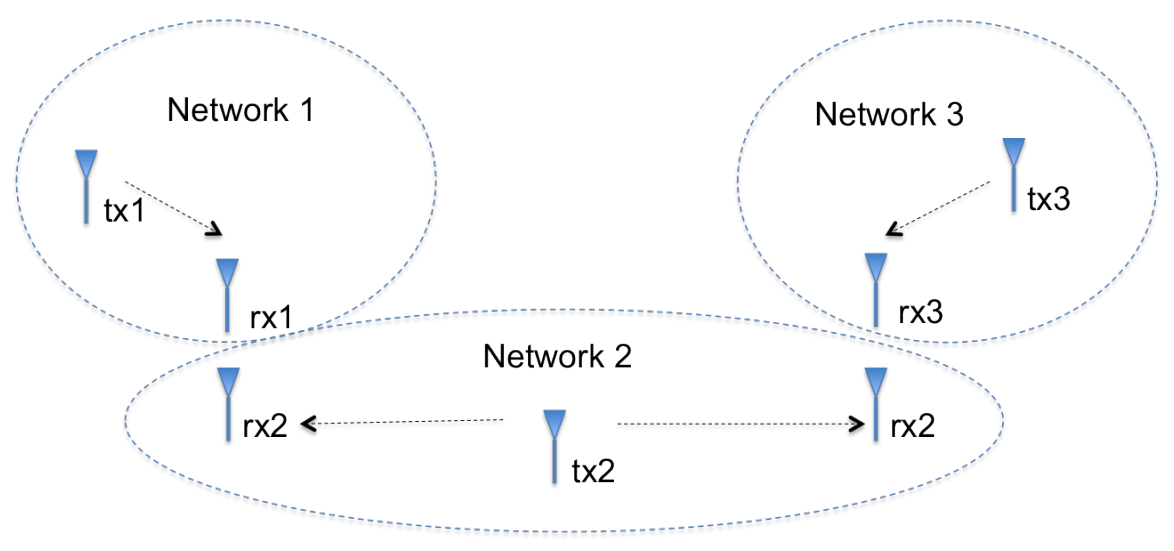

(a) Layout of Networks

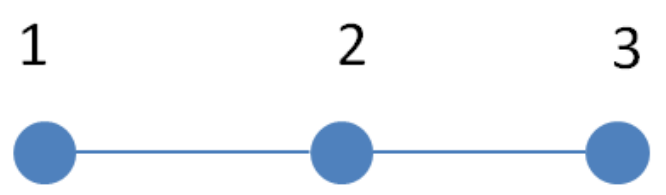

(b) Conflict Graph

Figure 4.1: Figure 4.1a shows a layout of networks that coexist in the same geographical area and Figure 4.1b shows its respective conflict graph.

allocated to networks 1 or 3 ; however, the channel can be allocated to networks 1 and 3 simultaneously as long as it is not allocated to network 2. This particularity of allocating a single channel to multiple users (network operators in this case) is commonly referred to as re-usability.

A compact representation of this type of constraints is obtained by means of a conflict graph $G(\mathcal{N}, E)$ where $\mathcal{N}$ is a set of nodes and $E \subseteq \mathcal{N} \times \mathcal{N}$ a set of undirected edges. Each node $v \in \mathcal{N}$ represents a network operator and each edge $(u, v) \in E$ represents a conflict among networks $u$ and $v$. In this case we say that networks $u$ and $v$ are neighbors or conflict with each other. Figure $4.1 \mathrm{~b}$ shows the conflict graph for the situation described above.

In this chapter, we propose Truthful Multichannel Auction (TMCA), an auction design where the spectrum owner is the auctioneer and the network operators are bidders; in the example above, we would have three bidders. TMCA is a sealed-bid multiple channel auction design with minimal computational demands that considers reusability and is shown to be incentive compatible in dominant strategies and individually rational. We further show expected revenue with this 
auction can be maximized by imposing a reserve price reminiscent of the standard optimal monopoly pricing rule.

\subsubsection{Literature Review}

Several authors have proposed auction formats for channel allocation considering private information and allocation constraints defined by means of a conflict graph, $[19,29,34,37,38]$. Zhou et al. proposed Veritas [37], a spectrum auction design that is shown to be incentive compatible and implementable in polynomial time under the assumption of constant marginal valuations. Zhou and Zheng proposed Trust [38], a double auction version of Veritas, that only allows bids for a single channel. $\mathrm{Xu}$ et al. extended the work of Zhou and Zheng by enabling bids for multiple channels [34]. Kash et al. proposed Satya [19], a truthful auction algorithm that allocates the right of contention for spectrum to users that can have either exclusive or sharing rights.

In all of the works mentioned above, bidders are assumed to have a constant marginal valuation for all channels. However, in reality, users are likely to have valuations that are marginally decreasing in the number of units allocated to them. A network, for example, may be interested in receiving two channels, one for high priority tasks and a second one for low priority tasks. If the network receives one channel (no matter which) then such channel is used for high priority tasks. If the network receives one additional channel, this second channel will be used for low priority tasks. Thus, the user's willingness to pay should decrease in the number of received channels.

To the best of our knowledge TMCA is the first auction design that is incentive compatible, individually rational and can be implemented in polynomial time, even when the marginal valuation of channels is not fixed. We also characterize a revenue maximizing TMCA design in which a reserve price per unit is determined.

This chapter is organized as follows: In section 4.2, we introduce a general auction framework considering reuse and explain how the VCG mechanism can be 
applied in this context. In section 4.3, we introduce TMCA and prove its properties (Incentive Compatibility, Individual Rationality and Polynomial Time). In section 4.4, we present some numerical experiments evaluating the computational time and efficiency of TMCA. We then conclude by presenting some conclusions and important remarks.

\subsection{Mathematical Framework}

In this section we introduce a generic model for "one shot" sealed bid multichannel auctions with multi-channel demand subject to interference constraints that are modeled by a conflict graph.

Let $\mathcal{N}=\{1, \cdots, N\}$ be the set of bidders (network operators). An auctioneer (spectrum owner) is to allocate a set $\mathcal{K}=\{1, \cdots, K\}$ of channels among bidders in $\mathcal{N}$ subject to the restrictions imposed by a conflict graph $G(\mathcal{N}, E)$. Note that $N$ and $K$ are the cardinality of the sets of bidders and channels respectively. An alternative way to present the information in the conflict graph $G(\mathcal{N}, E)$ is by means of a conflict matrix $\left(C M \in\{0,1\}^{N \times N}\right)$ defined as follows:

$$
C M(u, v)= \begin{cases}1 & \text { if }(u, v) \in E \\ 0 & \text { otherwise }\end{cases}
$$

Hence, users $u, v \in \mathcal{N}$ are in conflict iff $C M(u, v)=1$. Let $\mathbf{a} \in\{0,1\}^{N \times K}$ denote a channel allocation as follows:

$$
a_{n, k}= \begin{cases}1 & \text { if channel } k \in \mathcal{K} \text { is allocated to bidder } n \in \mathcal{N} \\ 0 & \text { otherwise }\end{cases}
$$

Definition: We say allocation a is feasible iff

$$
\mathbf{a}_{m, k}+\mathbf{a}_{n, k}<2
$$


for all $k \in \mathcal{K}$ and $m, n \in \mathcal{N}$ such that $C M(m, n)=1$. In what follows we shall denote by $\mathcal{A}$ the set of feasible allocations.

Each bidder has private information regarding his valuation of the channels. We assume that the valuation any user has for any set of channels he revceives only depends on the number of channels in the set. In other words, bidders are indifferent amongst channels. For example assume bidder 1 receives only one channel, so he is indifferent to that being channel 1 or channel $K$. Let $v_{n, k}>0$ be the marginal valuation of bidder $n$ for the $k$ th channel he receives. Let $\mathbf{v}_{n}=\left[v_{n, 1}, \ldots, v_{n, K}\right]$ denote the valuation vector of bidder $n \in \mathcal{N}$. As argued in the introduction, our auction is the first to consider users with valuations that are marginally not increasing in the number of channels receive. That is, we assume $v_{n, k}$ is not increasing in $k$ for all $n \in \mathcal{N}$. Note that $n$ 's utility given allocation $\mathbf{a} \in \mathcal{A}$ is:

$$
U_{n}\left(\mathbf{a}, \mathbf{v}_{n}\right)=\sum_{k=1}^{\left|\mathcal{K}_{n}(\mathbf{a})\right|} v_{n, k}
$$

where $\mathcal{K}_{n}(\mathbf{a})=\left\{k \in \mathcal{K}: a_{n, k}=1\right\}$ is the set of channels allocated to $n \in \mathcal{N}$ and $\left|\mathcal{K}_{n}(\mathbf{a})\right|$ its cardinality.

In a one-shot auction, each bidder is asked to report his valuation vector $\mathbf{v}_{n}=\left[v_{n, 1}, \ldots, v_{n, K}\right]$. In response the auctioneer finds a feasible allocation $\mathbf{a} \in \mathcal{A}$ and calculates payments $p_{n}$ for each $n \in \mathcal{N}$.

Let $\mathbf{b}_{n}=\left(b_{n, 1}, \ldots, b_{n, K}\right)$ be the bid of bidder $n \in \mathcal{N}$ (what $n$ claims to be his valuation $\mathbf{v}_{n}$ ). A one-shot auction design is completely described by the choice of an allocation and a payment rule. An allocation rule is a function $a(): \mathbb{R}^{N \times K} \rightarrow \mathcal{A}$, such that $a\left(\mathbf{b}_{1}, \ldots, \mathbf{b}_{N}\right)$ dictates the resultant allocation given bids $\left\{\mathbf{b}_{n}: n \in \mathcal{N}\right\}$. Payment rule for bidder $n \in \mathcal{N}$ is a function $p_{n}(): \mathbb{R}^{N \times K} \rightarrow \mathbb{R}$ such that $p_{n}\left(\mathbf{b}_{1}, \ldots, \mathbf{b}_{N}\right)$ dictates payment of bidder $n \in \mathcal{N}$.

Before introducing the TMCA design let us review how the well known VCG mechanism would be applied in this reusable channel setting. To fully describe VCG it will suffice to define the allocation and payment rules. In VCG the channel allocation rule (say $a^{*}(): \mathbb{R}^{N \times K} \rightarrow \mathcal{A}$ ) maximizes the social surplus given bids 
$\left\{b_{n}: n \in \mathcal{N}\right\}$, that is:

$$
a^{*}\left(\mathbf{b}_{1}, \ldots, \mathbf{b}_{N}\right) \in \underset{\mathbf{a} \in \mathcal{A}}{\operatorname{argmax}}\left[\sum_{n \in \mathcal{N}} U_{n}\left(\mathbf{a}, \mathbf{b}_{n}\right)\right]
$$

Payment of bidder $n \in \mathcal{N}$ (say $p_{n}^{*}(): \mathbb{R}^{N \times K} \rightarrow \mathbb{R}$ ) is the change imposed by $n$ 's participation on his competitors' utility. Let $\mathbf{b}_{-n}$ denote the bid of $n$ 's competitors and $a\left(\mathbf{b}_{n}, \mathbf{b}_{-n}\right)$ be the resultant allocation when bidder $n$ bids $\mathbf{b}_{n}$ and his competitors bid as stated in $\mathbf{b}_{-n}$. Note that $a^{*}\left(\mathbf{0}, \mathbf{b}_{-n}\right)$ is the resultant VCG allocation had bidder $n$ not participated in the auction. Hence the cost $n$ inflicts on his competitors is:

$$
\begin{aligned}
p_{n}^{*}\left(\mathbf{b}_{n}, \mathbf{b}_{-n}\right)= & \sum_{m \neq n} U_{m}\left(a^{*}\left(\mathbf{0}, \mathbf{b}_{-n}\right), \mathbf{b}_{m}\right) \\
& -\sum_{m \neq n} U_{m}\left(a^{*}\left(\mathbf{b}_{n}, \mathbf{b}_{-n}\right), \mathbf{b}_{m}\right)
\end{aligned}
$$

It is well-known that the VCG mechanism has the following properties:

- Incentive Compatible: For each bidder $n \in \mathcal{N}$ it is a dominant strategy to bid truthfully, i.e. to make $\mathbf{b}_{n}=\mathbf{v}_{n}$.

- Individually Rational: The net profit any user achieves by participating is never less than zero. This property is also known as the participation condition.

- Efficient: Its final outcome is socially efficient.

In VCG, bidding truthfully is a dominant strategy, and it guarantees the implementation of the socially efficient outcome (channel allocation in this case). Hence we say that VCG implements the socially efficient outcome in dominant strategies (see [24]). To implement VCG, however, the auctioneer needs to find the optimal channel allocation for several subsets of bidders in $\mathcal{N}$. Paralleling our reusable channel allocation problem with the chromatic number problem proves that this is 
an NP-complete problem (see Annex 4.6.1). Several authors argue this auction design is only of interest from a theoretical standpoint, [37], [29], [38] and [34]. From a practical standpoint, spectrum re-allocation in the short-run needs to be quick.

A second price approach is not trivial due to reusability. In order to highlight how re-usablility may affect the properties of the second price auction, consider the one channel problem depicted in Figure $4.1 \mathrm{~b}$ with associated valuations $v_{1,1}=10$, $v_{2,1}=12$ and $v_{3,1}=5$. Now consider the following options assuming truthful bidding:

- Consider a second price auction where the channel is allocated to the user with highest valuation, bidder 2 in this case. This is evidently not because the channel could be allocated to users 1 and 3 yielding a social welfare of 15 (instead of 12).

- Consider that the auctioneer modifies the allocation rule so that the channel is allocated to users 1 and 3. Note that even in this trivial example, it is not clear what the payments should be. A first intuition from second price auctions could be to charge each winner 12 (the highest offer from the non-winners). However, this would lead to negative profit for both winners. Splitting the payment of 12 will not work either because bidder 3 will end up with a negative profit. More elaborative rules like distributing the payment of 12 proportionally to their bids will generate incentives for non-truthful bidding. In the example above, bidder one could have bid 8 instead of 10 , still receiving the channel and paying a lower fraction of the total payment.

In what follows, we propose Truthful Multichannel Auction (TMCA), an auction design that can be implemented in polynomial time while ensuring that many of the desirable properties of the VCG mechanism still hold true. 


\subsection{Truthful Multi Channel Auction (TMCA)}

In this section we introduce TMCA, a "one-shot" sealed bid auction for the allocation of reusable channels. Later, we prove TMCA is incentive compatible, individually rational and can be executed in polynomial time.

A first feature of this auction design is an activity rule that imposes nonincreasing bids (i.e. $b_{n, k} \geq b_{n, k+1} \forall n \in \mathcal{N}, k \leq K$ ). This activity rule is imposed by the auctioneer, however this is a very reasonable requirement according to our assumption of marginally non-increasing valuations. In order to formally define the allocation and payment rules in TMCA, let us introduce the concept of the inverse residual supply curve for bidder $n \in \mathcal{N}$ which we shall denote by $\mathbf{s}_{n}=\left[s_{n, 1}, \cdots, s_{n, K}\right]$. Let $\mathcal{N}_{n}$ be the set of neighbors of $n$, that is $\mathcal{N}_{n}=\{m \in$ $\mathcal{N}: C M(m, n)=1\}$ and let $\mathcal{B}_{n}$ be a set containing all of their bid elements, i.e. $\mathcal{B}_{n}=\left\{b_{m, k}: m \in \mathcal{N}_{n}, k \leq K\right\}$. The curve $\mathbf{s}_{n}$ is a vector containing the $K$ largest elements of $\mathcal{B}_{n}$ in increasing order, that is:

$$
s_{n, k}=\mathcal{B}_{n}(K-k+1)
$$

where $k \leq K$ and $\mathcal{B}_{n}(k)$ denotes the $k$ th largest element in $\mathcal{B}_{n}$. If $\mathcal{B}_{n}=\emptyset$ then $\mathbf{s}_{n}=\mathbf{0}$.

Let us illustrate this through an example. Consider the case of two channels, three bidders and a conflict graph as shown in Fig. 4.1b. Assume bids $b_{1}=[10,0]$, $b_{2}=[9,7], b_{3}=[8,0]$ and . To find $\mathbf{s}_{\mathbf{1}}$, notice that bidder 2 is the unique neighbor of bidder 1, thus 9 and 7 are the largest and second largest bid elements among neighbors of bidder 1 . Therefore $s_{1,1}=7$, and $s_{1,2}=9$. In a similar way, we can find $\mathbf{s}_{\mathbf{2}}=[8,10]$ and $\mathbf{s}_{\mathbf{3}}=[7,9]$.

In TMCA $s_{n, k}$ will be: (i) the threshold that bidder $n$ has to exceed in $b_{n, k}$ to obtain a $k$ th channel and (ii) his payment for such a channel. Note that $s_{n, k}$ is non-decreasing and $b_{n, k}$ is non-increasing in $k$, Consequently $\left(b_{n, k}-s_{n, k}\right)$ is non-increasing in $k$. Consider a bidder with bid $\mathbf{b}_{n}$ and residual supply $\mathbf{s}_{n}$ as shown 
in Figure 4.2. In this case $b_{n, k}>s_{n, k}$ for $k \leq 5$ and $b_{n, k} \leq s_{n, k}$ for $k>5$. Hence bidder $n$ will receive 5 channels and his payment will be $p_{n}=\sum_{k=1}^{5} s_{n, k}$.

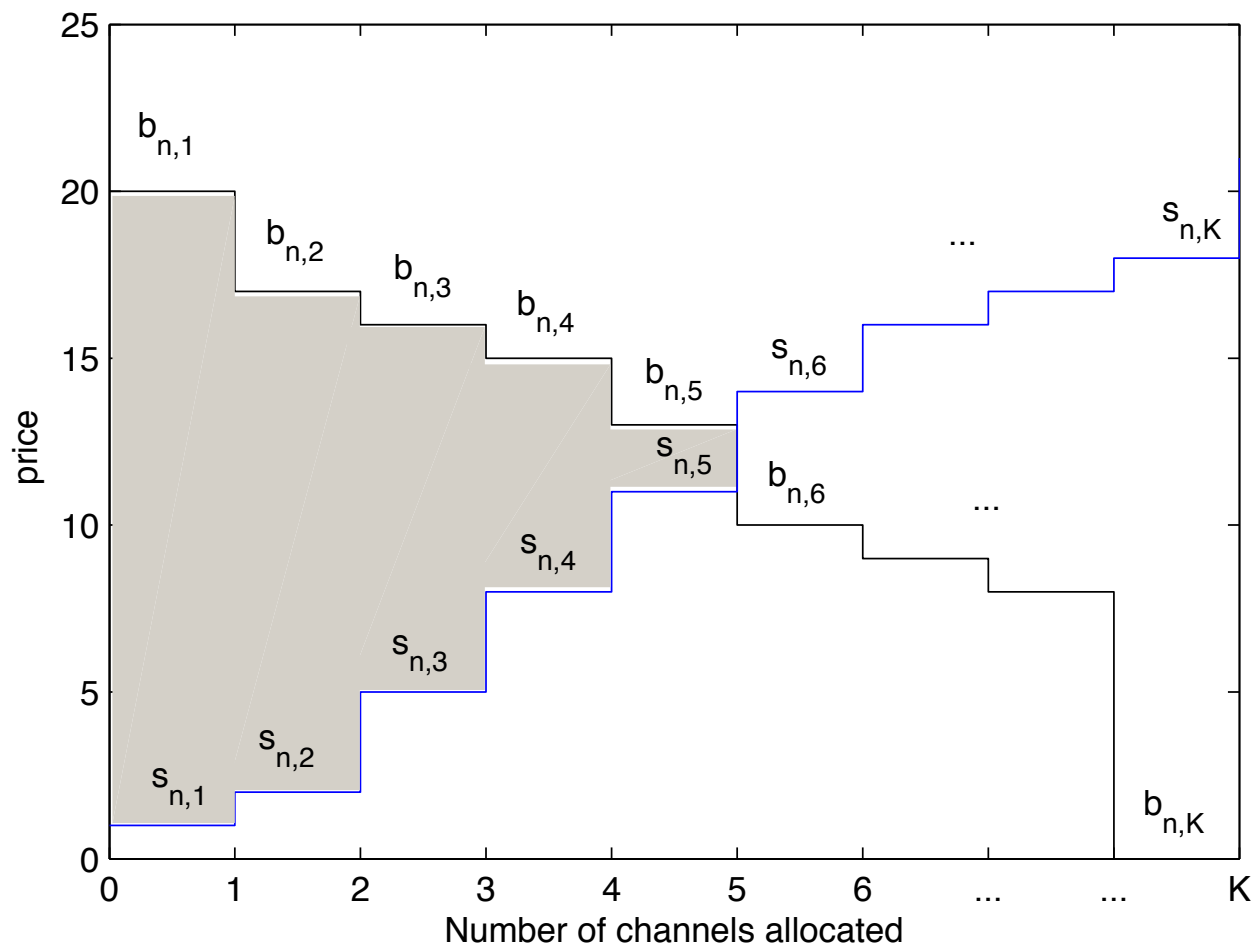

Figure 4.2: Example of non-increasing valuation and non-decreasing supply curve

We still have to define an allocation rule, say $a^{T M C A}: \mathbb{R}_{+}^{N K} \rightarrow \mathcal{A}$, such that given bids $\left\{\mathbf{b}_{n}: n \in \mathcal{N}\right\}$, allocation $\mathbf{a}=a^{T M C A}\left(\mathbf{b}_{1}, \ldots, \mathbf{b}_{N}\right)$ is consistent with all residual supply curves in the sense that:

$$
\sum_{k=1}^{K} \mathbf{a}_{n, k}=\sum_{k=1}^{K} \mathbf{1}_{\left\{b_{n, k}>s_{n, k}\right\}}
$$

For all $n \in \mathcal{N}$, where $\mathbf{1}_{\left\{b_{n, k}>s_{n, k}\right\}}=1$ if $b_{n, k}>s_{n, k}$ or 0 otherwise.

We propose an allocation, $a^{T M C A}: \mathbb{R}_{+}^{N K} \rightarrow \mathcal{A}$, that is performed in two phases. In the first phase we find a preallocation $\hat{\mathbf{a}} \in \mathcal{A}$ for which $\sum_{k=1}^{K} \hat{\mathbf{a}}_{n, k} \geq$ $\sum_{k=1}^{K} \mathbf{1}_{\left\{b_{n, k}>s_{n, k}\right\}}$ for all $n \in \mathcal{N}$. This phase is followed by an adjustment phase in which we arbitrarily deallocate channels until finding a final allocation a for which condition in Eq. 4.2 holds. 
- Preallocation: This phase makes iterative channel allocations considering all of the bid elements (i.e. all of the $b_{n, k}$ with $n \in \mathcal{N}$ and $k \leq K$ ) in a greedy fashion, giving higher priority to higher bid elements. It runs in iterations indexed by $\ell \in[1, \ldots, N \times K]$. Let $\mathcal{E}_{\ell}$ be the set of bid elements that have not been considered at iteration $\ell$. Naturally $\mathcal{E}_{1}$ contains all bid elements. That is, $\mathcal{E}_{1}=\left\{b_{n, k}: n \in \mathcal{N}, k \leq K\right\}$. At each iteration $\ell>0$, each bidder $n \in \mathcal{N}$ has a set of available channels, $\mathcal{A}_{n, \ell}$, which are available in the sense that they can be allocated to $n$ without interfering with any current allocation. Because we start with a null allocation $(\hat{\mathbf{a}}=\mathbf{0})$, all channels are initially available to all bidders, which means $\mathcal{A}_{n, 1}=\mathcal{K}$ for all $n \in \mathcal{N}$. We start by finding the largest bid element in $\mathcal{E}_{1}$, say $b_{n^{*}, k^{*}}$. In this case bidder $n^{*}$ has the largest bid element and he receives the first available channel in $\mathcal{A}_{n^{*}, 1}$, say $\hat{k}$. Note that in the next iteration channel $\hat{k}$ is not available for $n^{*}$ or his neighbors. Thus the sets of available channels has to be updated as follows: $\mathcal{A}_{m, 2}=\mathcal{A}_{m, 1} \backslash \hat{k}$ for $m \in\left\{n^{*}, \mathcal{N}_{n^{*}}\right\}$. For other bidders the set of available channels remains unchanged. Similarly bid $b_{n^{*}, k^{*}}$ does not need to be considered anymore, hence $\mathcal{E}_{2}=\mathcal{E}_{1} \backslash b_{n^{*}, k^{*}}$. The process is repeated until $\ell=N \times K$. This preallocation is formally described in the following pseudocode:

1. Initialize:

$$
\begin{aligned}
\mathcal{E}_{1} & =\left\{b_{n, k}: n \in \mathcal{N}, k \leq K\right\} \\
\mathcal{A}_{n, 1} & =\mathcal{K} \quad n \in \mathcal{N} \\
\hat{\mathbf{a}} & =\mathbf{0} ;
\end{aligned}
$$


2. Iterate: For $\ell=1, \ldots, N \times K$ :

$$
\begin{aligned}
\left(n^{*}, k^{*}\right) & =\arg \max \left\{b_{n, k}: b_{n, k} \in \mathcal{E}_{\ell}\right\} \\
\hat{k} & =\min \left\{k: k \in \mathcal{A}_{n^{*}, \ell}\right\} \\
\hat{\mathbf{a}}_{n^{*}, \hat{k}} & =1 ; \\
\mathcal{E}_{\ell+1} & =\mathcal{E}_{\ell} \backslash b_{n^{*}, k^{*}} \\
\mathcal{A}_{m, \ell+1} & =\mathcal{A}_{m, \ell} \backslash \hat{k} \quad \forall m \in\left\{n^{*}, \mathcal{N}_{n^{*}}\right\}
\end{aligned}
$$

where $\hat{k}=\emptyset$ whenever $\mathcal{A}_{n^{*}}=\emptyset$. In Appendix 4.6.2 we prove that the resultant allocation $\hat{\mathbf{a}}$, is such that for any $n \in \mathcal{N}$ and $k^{\prime} \leq K$ if $b_{n, k^{\prime}}>s_{n, k^{\prime}}$ then $\sum_{k=1}^{K} \hat{\mathbf{a}}_{n, k} \geq k^{\prime}$. Note this is equivalent to saying that for all $n \in \mathcal{N}$ :

$$
\sum_{k \in \mathcal{K}} \hat{\mathbf{a}}_{n, k} \geq \sum_{k \leq K} \mathbf{1}_{\left\{b_{n, k}>s_{n, k}\right\}}
$$

- Adjustment: Note that in the preallocation $\hat{\mathbf{a}}$, bidder $n \in \mathcal{N}$ may have received more than $\sum_{k \leq K} \mathbf{1}_{\left\{b_{n, k}>s_{n, k}\right\}}$ channels. The adjustment phase takes all of these "over-allocated" bidders and arbitrarily de-allocates as many channels as necessary to find an allocation a such that:

$$
\sum_{k \in \mathcal{K}} \mathbf{a}_{n, k}=\sum_{k \leq K} \mathbf{1}_{\left\{b_{n, k}>s_{n, k}\right\}}
$$

for all $n \in \mathcal{N}$

The preallocation phase is equivalent to sorting the $N K$ bid elements and finding the first available channel for each of the respective bidders; thus, its complexity is $O\left(N^{2} \log (N)\right)$ (assuming $\left.N>K\right)$. The adjustment phase requires $N K$ comparisons and possible deallocations; thus its complexity is $O\left(N^{2}\right)$. Notice that the allocation in TMCA is polynomial, but it is not guaranteed to be optimal. In section 4.4 we study the trade-off between efficiency and complexity for TMCA vs. VCG using numerical experiments. 
The two phases above define the mapping $a^{T M C A}: \mathbb{R}_{+}^{N K} \rightarrow \mathcal{A}$. Let as now define the payment rule $p_{n}^{T M C A}: \mathbb{R}_{+}^{N K} \rightarrow \mathbb{R}$. In TMCA, bidder $n \in \mathcal{N}$ is assessed a payment of:

$$
p_{n}\left(\mathbf{b}_{1}, \ldots, \mathbf{b}_{N}\right)=\sum_{k=1}^{\left|\mathcal{K}_{n}(\mathbf{a})\right|} s_{n, k}
$$

where $\mathbf{a}=a^{T M C A}\left(\mathbf{b}_{1}, \ldots, \mathbf{b}_{N}\right)$ is the resultant channel allocation, $\mathcal{K}_{n}(\mathbf{a})$ is the set of channels allocated to bidder $n$, and $\left|\mathcal{K}_{n}(\mathbf{a})\right|$ its cardinality.

Now we proceed to prove that TMCA is incentive compatible (IC) and individually rational (IR).

\subsubsection{Properties of TMCA}

Note that if bidder $n \in \mathcal{N}$ receives one channel his utility is $v_{n, 1}$, and his payment is $s_{n, 1}$, representing a profit of $\left(v_{n, 1}-s_{n, 1}\right)$. Similarly, the profit from the $k$ th channel received is $\left(v_{n, k}-s_{n, k}\right)$. Note also that residual supply curve $\mathbf{s}_{n}$ does not depend on n's bid, but only on his competitors' bids. Given n's competitors bids (hence residual supply $\mathbf{s}_{n}$ ), profit of bidder $n \in \mathcal{N}$, will be a function of his bid $\mathbf{b}_{n}$, and valuation $\mathbf{v}_{n}$, as follows:

$$
\pi_{n}\left(\mathbf{b}_{n}, \mathbf{v}_{n}\right)=\sum_{k \leq K}\left(v_{n, k}-s_{n, k}\right) \mathbf{1}_{\left\{b_{n, k}>s_{n, k}\right\}}
$$

Theorem 1: TMCA is incentive compatible in dominant strategies.

\section{Proof}

We need to prove that given any set of bids from $n$ 's competitors, say $\left\{\mathbf{b}_{m}: m \neq n\right\}$, it is a dominant strategy for $n \in \mathcal{N}$ to bid truthfully. Note that bids of $n$ 's competitors will completely define vector $\mathbf{s}_{n}$ and mapping $\pi_{n}(\cdot, \cdot)$. Hence, it will suffice to show that:

$$
\mathbf{v}_{n} \in \underset{\mathbf{b}_{n}}{\operatorname{argmax}}\left\{\pi_{n}\left(\mathbf{b}_{n}, \mathbf{v}_{n}\right)\right\}
$$


By contradiction, assume there exists some $\mathbf{b}_{n} \neq \mathbf{v}_{n}$ such that

$$
\pi_{n}\left(\mathbf{v}_{n}, \mathbf{v}_{n}\right)<\pi_{n}\left(\mathbf{b}_{n}, \mathbf{v}_{n}\right)
$$

or what is the same

$$
\sum_{k \leq K}\left(v_{n, k}-s_{n, k}\right) \mathbf{1}_{\left\{v_{n, k}>s_{n, k}\right\}}<\sum_{k \leq K}\left(v_{n, k}-s_{n, k}\right) \mathbf{1}_{\left\{b_{n, k}>s_{n, k}\right\}}
$$

Note that all terms in the sum on the left are positive. Hence, there must exist some $\hat{k} \leq K$ such that $\left(v_{n, \hat{k}}-s_{n, \hat{k}}\right)>0$ that is in the right hand side sum but not in the left had side sum. That is $\mathbf{1}_{\left\{b_{n, \hat{k}}>s_{n, \hat{k}}\right\}}=1$ and $\mathbf{1}_{\left\{v_{n, \hat{k}}>s_{n, \hat{k}}\right\}}=0$. However note that $\mathbf{1}_{\left\{v_{i, \hat{k}}>s_{i, \hat{k}}\right\}}=0$ implies $\left(v_{i, \hat{k}}-s_{i, \hat{k}}\right) \leq 0$, a contradiction to $\left(v_{n, \hat{k}}-s_{n, \hat{k}}\right)>0$.

\section{Theorem 2 TMCA is Individually Rational}

\section{Proof}

Note that after having truthfully participated in the TMCA, profit of bidder $n \in \mathcal{N}$ is:

$$
\sum_{k \in \mathcal{K}}\left(b_{n, k}-s_{n, k}\right) \mathbf{1}_{\left\{b_{n, k}>s_{n, k}\right\}} \geq 0
$$

Note that the properties highlighted in Theorems 1 and 2 are also held in VCG. However TMCA can be performed in polynomial time. This of course, is done at the expense of social efficiency. In section 4.4, we compare efficiency and computing time for TMCA and VCG through simulations. Before that let us explore the use of a reserve price, a common practice usually intended to increase the revenue of the seller (at the expense of efficiency).

\subsubsection{TMCA Optimal Reserve Price}

In this section, we look for an optimal reserve price that maximizes the expected seller's revenue. Maximizing seller's revenue (also known as optimal mechanism 
design) was initially proposed by Myerson [26] and Riley and Samuelson [28]. Existing theory about optimal mechanism design has not considered re-usability; thus, it does not necessarily apply to our problem. Similarly, literature that considers re-usability has focused on efficiency and has not studied reserve prices. In this section we study optimal mechanism design, considering reusability and assuming valuations of bidder $n \in \mathcal{N}$ are of the form:

$$
v_{n, k}=\left\{\begin{array}{lll}
v_{n} & \text { for } & k \leq d \\
0 & \text { for } & k>d
\end{array}\right.
$$

for some $d \in\{1, \ldots, K\}$ and $v_{n}>0$. We also assume valuations $\left\{v_{n}: n \in \mathcal{N}\right\}$ are i.i.d random variables with $\mathrm{CDF} F$ and bounded, positive, non-atomic pdf $f$ with support $[0, \bar{b}]$. Note that setting a reserve price will change the residual supply curves $\left\{\mathbf{s}_{n}: n \in \mathcal{N}\right\}$. Let $b_{n, k}^{0}$ denotes the reserve price the seller imposes on bidder $n \in \mathcal{N}$ for the $k$ th channel he receives. According to our previous analysis we shall assume $b_{n, k}^{0}$ is non-decreasing in $k$ for all $n \in \mathcal{N}$. Note that given reserve prices $\left\{b_{n, k}^{0}: n \leq N, k \leq K\right\}$, residual prices should be redefined as $s_{n, k}=\max \left(\hat{s}_{n, k}, b_{n, k}^{0}\right)$ for all $n \leq N$ and $k \leq K$, where $\hat{\mathbf{s}}_{n}=\left[\hat{s}_{n, k}, \ldots, \hat{s}_{n, K}\right]$ is the supply curve for bidder $n$ in the absence of reserve prices (i.e. as defined in section 4.3).

Note that Theorems 1 and 2 prove Incentive Compatibility and Individual Rationality for a generic bidder $n \in \mathcal{N}$ given any supply curve $\mathbf{s}_{n}$. Hence these results hold true if we modify the supply curve to consider reserve prices. Given incentive compatibility, bids are

$$
b_{n, k}=\left\{\begin{array}{lll}
v_{n} & \text { for } & k \leq d \\
0 & \text { for } & k>d
\end{array}\right.
$$

Although the auctioneer does not know the valuations a priori, we assume he knows their common pdf $f$. The following theorem describes the reserve prices that maximize his expected revenue. 


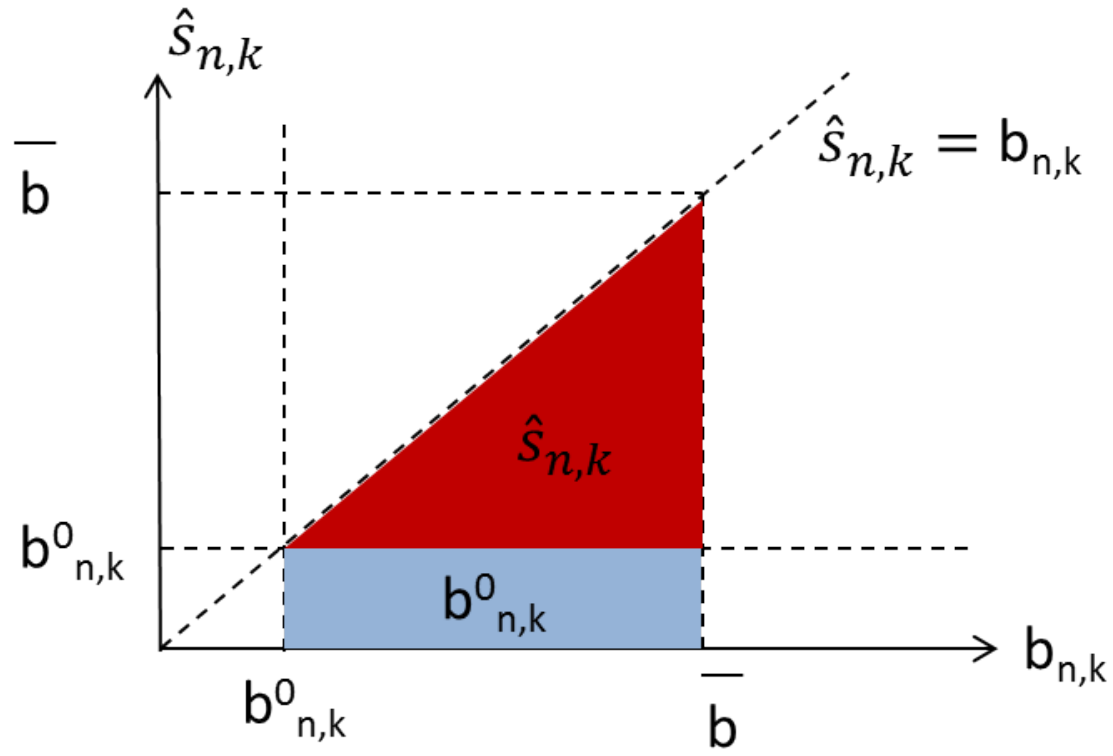

Figure 4.3: $p_{n, k}$ for all possible values of $b_{n, k}^{0}$ and $\hat{s}_{n, k}$

Theorem 3: In TMCA, the seller maximizes expected revenue by setting a uniform reserve price, $b_{0} \in(0, \bar{b})$, which is implicitly defined by

$$
b_{0}=\frac{1}{\frac{f\left(b_{0}\right)}{1-F\left(b_{0}\right)}}
$$

Proof Let $p_{n, k}$ be the price charged to bidder $n$ for the $k$ th channel he receives. Note that

$$
p_{n, k}\left(\hat{s}_{n, k}, b_{n, k}, b_{n, k}^{0}\right)= \begin{cases}\hat{s}_{n, k} & \text { if } b_{n, k}>\hat{s}_{n, k}>b_{n, k}^{0} \\ b_{n, k}^{0} & \text { if } b_{n, k}>b_{n, k}^{0}>\hat{s}_{n, k} \\ 0 & \text { Otherwise }\end{cases}
$$

Figure 4.3 shows $p_{n, k}$ for all possible values of $\hat{s}_{n, k}$ and $b_{n, k}$. The red area corresponds to $b_{n, k}>\hat{s}_{n, k}>b_{n, k}^{0}$ in which $p_{n, k}=\hat{s}_{n, k}$. The blue area corresponds to $b_{n, k}>b_{n, k}^{0}>\hat{s}_{n, k}$ in which $p_{n, k}=b_{n, k}^{0}$. For all other possibilities $p_{n, k}=0$.

Revenue of the auctioneer is the sum of the payments of all bidders:

$$
R=\sum_{n=1}^{N} \sum_{k=1}^{d} p_{n, k}
$$

Note that we are interested in $p_{n, k}$ for $k \leq d$ in which case $b_{n, k}=v_{n}$. This is 
due to the rationality of bidders. Furthermore, given reserve prices $\left(\left\{b_{n, k}^{0}: n \in\right.\right.$ $\mathcal{N}, \quad k \leq K\}), p_{n, k}$ is a random variable that depends only on the values of $v_{n, k}$ and $\hat{s}_{n, k}$.

We want to maximize the expected value of the auctioneer's revenue $R$. Let us start by showing that there exists a reserve price, $b_{n, k}^{0} \in[0, \bar{b}]$, that maximizes $E\left[p_{n, k}\right]$. Later we prove that such value can not be 0 or $\bar{b}$. Furthermore this value does not depend on $n$ or $k$, for simplicity we call it $b_{0}$. The fact that such value is interior implies the necessary condition stated in the theorem.

Notice that $v_{n}$ and $\hat{s}_{n, k}$ are two independent random variables. Thus, their joint probability density function can be stated as:

$$
f_{s, v}\left(s^{\prime}, v^{\prime}\right)=f_{s}\left(s^{\prime}\right) f\left(v^{\prime}\right)
$$

Where $f_{s}$ and is the density function of $\hat{s}_{n, k}$ and $f$ is the density function of $v_{n}$.

Let $\eta_{n}$ be the number of neighbors of $n$ (or conflict degree of $n$ in the conflict graph). In order to find the expected value of $p_{n, k}$ as a function of $b_{n, k}^{0}$, consider the following two cases:

1. $\hat{s}_{n, k}=0$ :

In this case $p_{n, k}=b_{n, k}^{0}$ if $b_{n, k}^{0}<v_{n}$ or 0 otherwise. Hence:

$$
\begin{aligned}
E\left[p_{n, k} \mid \hat{s}_{n, k}=0\right] & =\operatorname{Pr}\left(v_{n}>b_{n, k}^{0}\right) b_{n, k}^{0} \\
& =\left(1-F\left(b_{n, k}^{0}\right)\right) b_{n, k}^{0}
\end{aligned}
$$

2. $\hat{s}_{n, k}>0$ :

In this case $\eta_{n}-1 \geq\lfloor(K-k) / d\rfloor$. Note that the probability of having $\hat{s}_{n, k} \in\left(s^{\prime}, s^{\prime}+\delta s\right)$, with $\delta s \rightarrow 0$, is the probability of having:

- $\lfloor(K-k) / d\rfloor$ neighbors with $b_{j}>s^{\prime}+\delta s$

- One neighbor with $b_{j} \in\left(s^{\prime}, s^{\prime}+\delta s\right)$ and 
- $\eta_{n}-\lfloor(K-k) / d\rfloor-1$ neighbors with $b_{j}<s^{\prime}$

Thus, the density function of $\hat{s}_{n, k}$ is:

$$
f_{s}\left(s^{\prime}\right)=\eta_{n}\left(\begin{array}{c}
\eta_{n}-1 \\
\lfloor(K-k) / d\rfloor
\end{array}\right) f\left(s^{\prime}\right)\left(1-F\left(s^{\prime}\right)\right)^{\lfloor(K-k) / d\rfloor}\left(F\left(s^{\prime}\right)\right)^{\eta_{n}-\lfloor(K-k) / d\rfloor-1}
$$

Now, using equation (4.5) we get:

$$
\begin{aligned}
E\left[p_{n, k} \mid \hat{s}_{n, k}>0\right] & \\
= & \int_{0}^{\bar{b}} \int_{0}^{\bar{b}} f_{s, v}(x, y) p_{n, k}(x, y) d x d y \\
= & b_{n, k}^{0} \int_{b_{n, k}^{0}}^{\bar{b}} \int_{0}^{b_{n, k}^{0}} f_{s}(x) f(y) d x d y \\
& +\int_{b_{n, k}^{0}}^{\bar{b}} \int_{b_{n, k}^{0}}^{y} x f_{s}(x) f(y) d x d y \\
= & b_{n, k}^{0}\left(1-F\left(b_{n, k}^{0}\right)\right) F_{s}\left(b_{n, k}^{0}\right) \\
& +\int_{b_{n, k}^{0}}^{\bar{b}} \int_{b_{n, k}^{0}}^{y} x f_{s}(x) f(y) d x d y
\end{aligned}
$$

Our objective is to maximize $E\left[p_{n, k}\right]$ for all $n \in \mathcal{N}, k \leq d$. Note that boundedness of $f$ implies boundedness of $E\left[p_{n, k}\right]$ for all $n \in \mathcal{N}, k \leq d$. Therefore, by compactness, there exist an optimal $b_{n, k}^{0}$ in $[0, \bar{b}]$.

We proceed to find $\frac{\partial}{\partial b_{n, k}^{0}} E\left[p_{n, k}\right]$ for both cases, $\hat{s}_{n, k}=0$ and $\hat{s}_{n, k}>0$ :

1. For $s_{n, k}=0$ we have:

$$
\frac{\partial}{\partial b_{n, k}^{0}} E\left[p_{n, k} \mid \hat{s}_{n, k}=0\right]=1-F\left(b_{n, k}^{0}\right)-b_{n, k}^{0} f\left(b_{n, k}^{0}\right)
$$


2. For $s_{n, k}>0$ we can approach both terms in Eq. 4.7 separately as follows:

$$
\begin{aligned}
& \frac{\partial}{\partial b_{n, k}^{0}}\left(b_{n, k}^{0}\left(1-F\left(b_{n, k}^{0}\right)\right) F_{s}\left(b_{n, k}^{0}\right)\right) \\
& =\left(1-F\left(b_{n, k}^{0}\right)\right) F_{s}\left(b_{n, k}^{0}\right) \\
& \quad+b_{n, k}^{0}\left[\left(1-F\left(b_{n, k}^{0}\right)\right) f_{s}\left(b_{n, k}^{0}\right)-f\left(b_{n, k}^{0}\right) F_{s}\left(b_{n, k}^{0}\right)\right]
\end{aligned}
$$

and

$$
\begin{aligned}
\frac{\partial}{\partial b_{n, k}^{0}} & \left(\int_{b_{n, k}^{0}}^{\bar{b}} \int_{b_{n, k}^{0}}^{y} x f_{s}(x) f(y) d x d y\right) \\
= & \int_{b_{n, k}^{0}}^{\bar{b}} \frac{\partial}{\partial b_{n, k}^{0}}\left(\int_{b_{n, k}^{0}}^{y} x f_{s}(x) f(y) d x\right) d y \\
& -\int_{b_{n, k}^{0}}^{b_{n, k}^{0}} x f_{s}(x) f\left(b_{n, k}^{0}\right) d x \\
= & \int_{b_{n, k}^{0}}^{\bar{b}} \frac{\partial}{\partial b_{n, k}^{0}}\left(\int_{b_{n, k}^{0}}^{y} x f_{s}(x) f(y) d x\right) d y \\
= & -\int_{b_{n, k}^{0}}^{\bar{b}} b_{n, k}^{0} f_{s}\left(b_{n, k}^{0}\right) f(y) d y \\
= & -b_{n, k}^{0} f_{s}\left(b_{n, k}^{0}\right)\left(1-F\left(b_{n, k}^{0}\right)\right)
\end{aligned}
$$

Notice we applied Leibniz's rule twice in (4.9). It follows from Eq. (4.7), (4.8), and (4.9) that:

$$
\begin{aligned}
& \frac{\partial}{\partial b_{n, k}^{0}} E\left[p_{n, k} \mid \hat{s}_{n, k}>0\right] \\
& \quad=F_{s}\left(b_{n, k}^{0}\right)\left(1-F\left(b_{n, k}^{0}\right)-b_{n, k}^{0} f\left(b_{n, k}^{0}\right)\right)
\end{aligned}
$$

Note that the optimal reserve price can not be 0 . For the case $\hat{s}_{n, k}>0$ a reserve price of $b_{n, k}^{0}=0$ has no effect, for the case $\hat{s}_{n, k}=0$ we have:

$$
\left.\frac{\partial E\left[p_{n, k}\right]}{\partial b_{n, k}^{0}}\right|_{b_{n, k}^{0} \downarrow 0}=1>0
$$


Furthermore, notice that $\left.E\left[p_{n, k}\right]\right|_{b_{n, k}^{0}=\bar{b}}=0$. Thus, the optimal price has to reside in $(0, \bar{b})$.

Because the solution is interior, a necessary condition for optimality is $\frac{\partial}{\partial b_{n, k}^{0}} E\left[p_{n, k}\right]=$ 0 , which in both cases implies $1-F\left(b_{n, k}^{0}\right)-b_{n, k}^{0} f\left(b_{n, k}^{0}\right)=0$. This proves that the optimal (interior) reserve price will be as stated in the theorem, where we used $b_{0}$ (instead of $b_{n, k}^{0}$ ) to highlight the fact that it does not depend on $n \in \mathcal{N}$ nor $k \leq d$.

In the following section, we compare TMCA with VCG by way of numerical experiments. We also verify the result of Theorem 3 assuming valuations uniformly distributed in $(0,1)$. For this case, note that Theorem 3 suggests a reserve price $b_{0}=0.5$ in order to maximize revenue of the auctioneer.

\subsection{Numerical Experiments and Analysis}

We consider an ad-hoc topology testbed, which has been widely used by other authors dealing with this problem. In this testbed bidders are randomly located in a $1 \mathrm{x} 1$ square. If the distance between two of them is less than some critical distance (0.1 is commonly used), these bidders conflict with each other. Note this will generate completely random topologies. We study performance of TMCA vs. VCG in this testbed with $N \in\{4,6,8,10\}$ bidders and $K \in\{2,4\}$ channels.For each configuration of $N, K$ and $b_{0}$, experiments were run for 200 times; graphs and tables show the average results.

The first numerical experiment illustrates the result of Theorem 3. Fig. 4.4 shows revenue of the auctioneer when auctioning 4 channels, assuming bids as described in Eq. 4.4 with valuations uniformly distributed in $(0,1)$. Not surprisingly, revenue is proportional to the number of bidders $N$ and to the number of demanded channels $d$. According to Theorem 3 , if bids are uniform in $(0,1)$ the auctioneer maximizes revenue by setting a reserve price of $b_{0}=0.5$. Note that results in Fig. 4.4 support this result. 

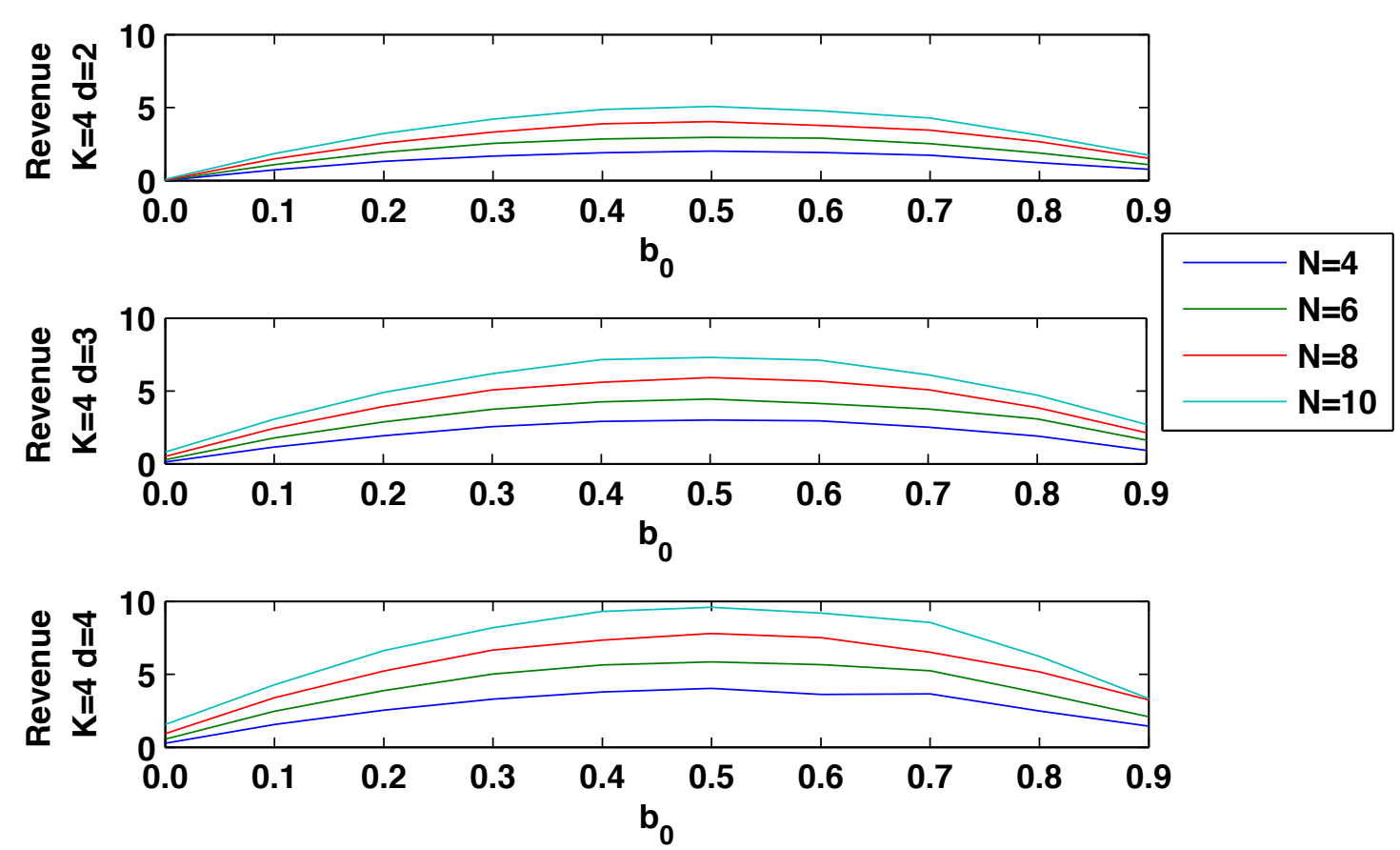

Figure 4.4: Revenue of auctioneer for different values of $b_{0}$. From top to bottom we show results for $d=2, d=3$ and $d=4$. Each subfigure considers $N \in\{4,6,8,10\}$ bidders.

The following experiments compare TMCA and VCG. We are especially interested in measuring speed and social welfare. We do not compare TMCA to other mechanisms mentioned above because none of them support variable marginal valuations. We study two type of networks: (i)Networks with low density of users (using a critical distance of 0.1) and (ii) Networks with low density of users (using a critical distance of 0,3$)$. Networks with high density of users could have been studied by simply increasing the number of users $N$. However increasing $N$ beyond 10 resulted computationally too demanding for VCG.

\subsubsection{Low Density Mesh}

Tables 4.1 and 4.2 compare the computational time of TMCA vs. VCG for $K=2$ and $K=4$ channels respectively. Figure 4.5 summarizes the results with reserve prices $b_{0}=0$ and $b_{0}=0.5$. Experiments were run using Intel Xeon X5550 @2.67GHz, CPU MHz: 2667, Cache Size: 8192K. CPU time was measured using 


\begin{tabular}{|l|l|l|l|l||l|l|l|l|}
\hline & \multicolumn{4}{|c|}{ TMCA } & \multicolumn{4}{c|}{ VCG } \\
\hline$b_{0} \backslash \mathrm{N}$ & 2 & 4 & 6 & 8 & 2 & 4 & 6 & 8 \\
\hline 0 & 0.005 & 0.005 & 0.006 & 0.008 & 0.049 & 0.115 & 0.296 & 0.486 \\
\hline 0.1 & 0.002 & 0.004 & 0.006 & 0.007 & 0.047 & 0.144 & 0.280 & 0.486 \\
\hline 0.2 & 0.002 & 0.004 & 0.005 & 0.007 & 0.047 & 0.142 & 0.269 & 0.481 \\
\hline 0.3 & 0.002 & 0.003 & 0.005 & 0.006 & 0.048 & 0.136 & 0.270 & 0.488 \\
\hline 0.4 & 0.001 & 0.003 & 0.005 & 0.005 & 0.048 & 0.138 & 0.281 & 0.483 \\
\hline 0.5 & 0.001 & 0.002 & 0.004 & 0.004 & 0.048 & 0.140 & 0.275 & 0.477 \\
\hline 0.6 & 0.002 & 0.002 & 0.003 & 0.004 & 0.047 & 0.137 & 0.281 & 0.467 \\
\hline 0.7 & 0.001 & 0.002 & 0.003 & 0.004 & 0.048 & 0.140 & 0.279 & 0.487 \\
\hline 0.8 & 0.001 & 0.001 & 0.001 & 0.003 & 0.047 & 0.140 & 0.273 & 0.491 \\
\hline 0.9 & 0.001 & 0.001 & 0.001 & 0.001 & 0.046 & 0.139 & 0.272 & 0.493 \\
\hline
\end{tabular}

Table 4.1: CPU time of TMCA and VCG for $K=2$ channels

\begin{tabular}{|l|l|l|l|l||l|l|l|l|}
\hline & \multicolumn{4}{|c|}{ TMCA } & \multicolumn{4}{c|}{ VCG } \\
\hline$b_{0} \backslash \mathrm{N}$ & 2 & 4 & 6 & 8 & 2 & 4 & 6 & 8 \\
\hline 0 & 0.005 & 0.008 & 0.013 & 0.016 & 0.052 & 0.180 & 0.543 & 1.227 \\
\hline 0.1 & 0.004 & 0.007 & 0.012 & 0.016 & 0.086 & 0.274 & 0.646 & 1.089 \\
\hline 0.2 & 0.004 & 0.007 & 0.011 & 0.015 & 0.090 & 0.257 & 0.640 & 1.386 \\
\hline 0.3 & 0.004 & 0.007 & 0.010 & 0.014 & 0.094 & 0.266 & 0.676 & 1.243 \\
\hline 0.4 & 0.003 & 0.005 & 0.009 & 0.012 & 0.097 & 0.290 & 0.707 & 1.341 \\
\hline 0.5 & 0.003 & 0.005 & 0.007 & 0.011 & 0.099 & 0.295 & 0.701 & 1.477 \\
\hline 0.6 & 0.003 & 0.004 & 0.007 & 0.008 & 0.101 & 0.306 & 0.727 & 1.246 \\
\hline 0.7 & 0.002 & 0.004 & 0.005 & 0.007 & 0.096 & 0.298 & 0.672 & 1.252 \\
\hline 0.8 & 0.002 & 0.002 & 0.003 & 0.004 & 0.085 & 0.302 & 0.647 & 1.236 \\
\hline 0.9 & 0.001 & 0.002 & 0.002 & 0.003 & 0.086 & 0.288 & 0.664 & 1.222 \\
\hline
\end{tabular}

Table 4.2: CPU time of TMCA and VCG for $K=4$ channels

the cputime Matlab built-in function.

Notice how computing time for VCG grows exponentially as the number of nodes increases. This makes TMCA a more desirable mechanism when time plays an important role. We already know that such benefit will have a toll in social surplus. Tables 4.3 and 4.5 show social surplus and revenue of the auctioneer for TMCA and VCG when $K=2$ channels. Similarly, Tables 4.4 and 4.6 do the same for $K=4$ channels. These tables are summarized in Fig. 4.6.

Notice that results in efficiency and revenue are virtually the same in VCG and TMCA. The maximum efficiency loss revealed in our experiments was of $0.89 \%$ (for $N=10$ users and a reserve price of $b_{0}=0.2$ ). Yet, TMCA showed reductions 

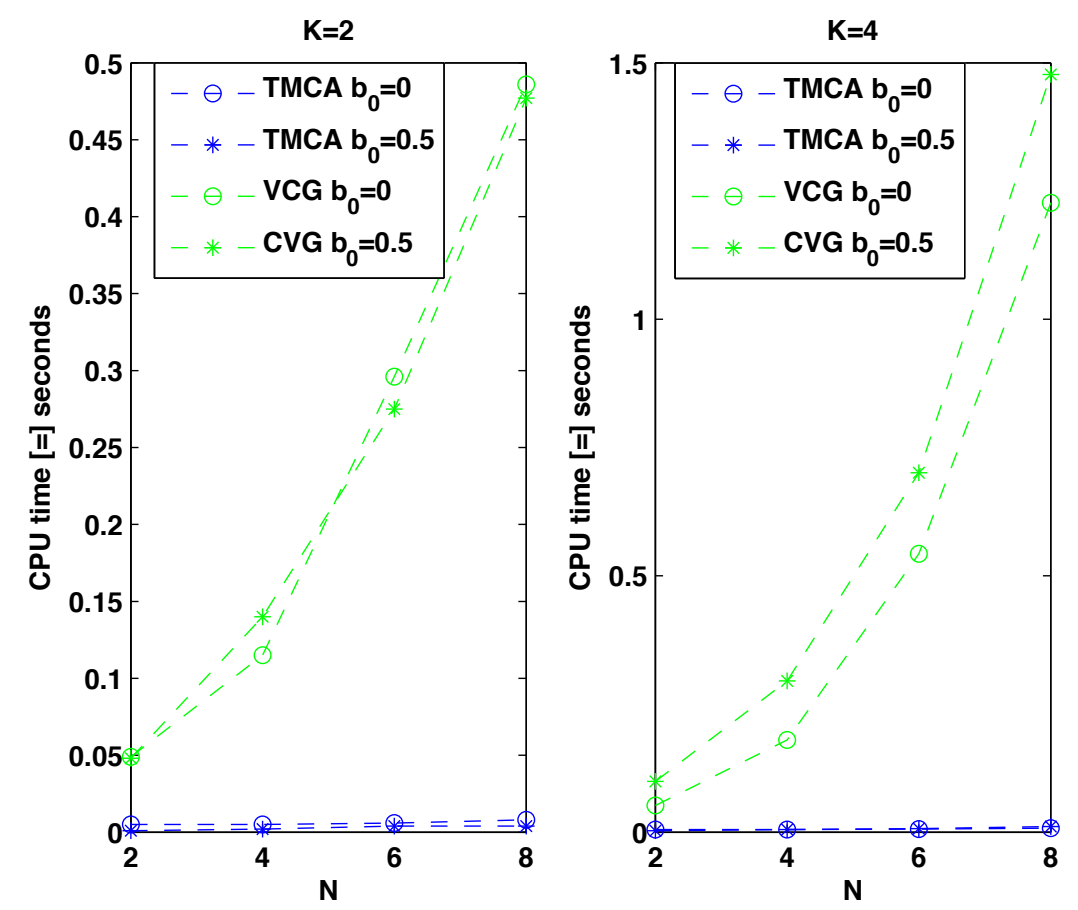

Figure 4.5: Exponential vs polynomial CPU time

\begin{tabular}{|l|l|l|l|l||l|l|l|l|}
\hline & \multicolumn{5}{|c||}{ TMCA } & \multicolumn{4}{c|}{ VCG } \\
\hline$b_{0} \backslash \mathrm{N}$ & 4 & 6 & 8 & 10 & 4 & 6 & 8 & 10 \\
\hline 0 & 3.793 & 5.415 & 7.440 & 9.279 & 3.793 & 5.415 & 7.445 & 9.279 \\
\hline 0.1 & 3.822 & 5.658 & 7.379 & 8.877 & 3.822 & 5.658 & 7.392 & 8.907 \\
\hline 0.2 & 3.865 & 5.845 & 7.022 & 8.878 & 3.865 & 5.845 & 7.046 & 8.958 \\
\hline 0.3 & 3.648 & 5.139 & 6.600 & 8.718 & 3.648 & 5.139 & 6.627 & 8.738 \\
\hline 0.4 & 3.284 & 4.944 & 6.431 & 7.703 & 3.284 & 4.944 & 6.442 & 7.716 \\
\hline 0.5 & 3.035 & 4.130 & 5.713 & 7.336 & 3.035 & 4.130 & 5.713 & 7.336 \\
\hline 0.6 & 2.325 & 3.970 & 5.365 & 6.335 & 2.325 & 3.970 & 5.365 & 6.375 \\
\hline 0.7 & 1.779 & 2.984 & 4.222 & 4.969 & 1.779 & 2.984 & 4.222 & 5.004 \\
\hline 0.8 & 1.506 & 2.148 & 3.073 & 3.375 & 1.506 & 2.148 & 3.073 & 3.375 \\
\hline 0.9 & 0.628 & 0.993 & 1.306 & 1.872 & 0.628 & 0.993 & 1.306 & 1.872 \\
\hline
\end{tabular}

Table 4.3: Social Surplus of TMCA and VCG for $K=2$ channels

in CPU time of the order of $10^{2}$.

There seems to be a tendency of increasing inefficiency, hence we study scenarios with higher density of users by using a critical distance of 0.3 instead of 0.1

Tables 4.7 and 4.8 compare the computational time of TMCA vs. VCG for $K=2$ and $K=4$ respectively. Figure 4.7 summarizes the results with reserve prices $b_{0}=0$ and $b_{0}=0.5$. 


\begin{tabular}{|l|l|l|l|l||l|l|l|l|}
\hline & \multicolumn{4}{|c|}{ TMCA } & \multicolumn{4}{c|}{ VCG } \\
\hline$b_{0} \backslash \mathrm{N}$ & 4 & 6 & 8 & 10 & 4 & 6 & 8 & 10 \\
\hline 0 & 7.398 & 11.588 & 15.424 & 18.559 & 7.398 & 11.604 & 15.448 & 18.578 \\
\hline 0.1 & 7.520 & 11.364 & 14.910 & 17.904 & 7.535 & 11.375 & 14.931 & 18.016 \\
\hline 0.2 & 7.427 & 11.472 & 14.802 & 18.246 & 7.427 & 11.472 & 14.851 & 18.269 \\
\hline 0.3 & 7.284 & 10.705 & 13.888 & 16.435 & 7.284 & 10.705 & 13.915 & 16.542 \\
\hline 0.4 & 6.623 & 10.105 & 12.921 & 15.860 & 6.633 & 10.105 & 12.933 & 15.930 \\
\hline 0.5 & 5.967 & 8.842 & 11.912 & 14.693 & 5.967 & 8.842 & 11.911 & 14.715 \\
\hline 0.6 & 5.463 & 7.416 & 10.368 & 12.990 & 5.463 & 7.416 & 10.368 & 12.990 \\
\hline 0.7 & 4.216 & 6.211 & 7.957 & 9.355 & 4.216 & 6.211 & 7.957 & 9.372 \\
\hline 0.8 & 2.782 & 4.110 & 5.702 & 7.615 & 2.782 & 4.110 & 5.702 & 7.632 \\
\hline 0.9 & 1.367 & 2.035 & 2.995 & 3.628 & 1.367 & 2.035 & 2.995 & 3.628 \\
\hline
\end{tabular}

Table 4.4: Social Surplus of TMCA and VCG for $K=4$ channels

\begin{tabular}{|l|l|l|l|l||l|l|l|l|}
\hline & \multicolumn{4}{|c||}{ TMCA } & \multicolumn{4}{c|}{ VCG } \\
\hline$b_{0} \backslash \mathrm{N}$ & 4 & 6 & 8 & 10 & 4 & 6 & 8 & 10 \\
\hline 0 & 0.081 & 0.286 & 0.446 & 0.778 & 0.081 & 0.286 & 0.441 & 0.765 \\
\hline 0.1 & 0.767 & 1.287 & 1.618 & 2.096 & 0.767 & 1.275 & 1.616 & 2.065 \\
\hline 0.2 & 1.286 & 2.032 & 2.579 & 3.199 & 1.286 & 2.032 & 2.575 & 3.194 \\
\hline 0.3 & 1.652 & 2.398 & 3.186 & 4.187 & 1.652 & 2.398 & 3.210 & 4.141 \\
\hline 0.4 & 1.887 & 2.853 & 3.707 & 4.548 & 1.887 & 2.853 & 3.712 & 4.545 \\
\hline 0.5 & 1.992 & 2.916 & 3.965 & 4.939 & 1.992 & 2.716 & 3.865 & 4.928 \\
\hline 0.6 & 1.776 & 2.982 & 4.027 & 4.790 & 1.776 & 2.981 & 4.027 & 4.826 \\
\hline 0.7 & 1.456 & 2.455 & 3.472 & 4.113 & 1.456 & 2.455 & 3.472 & 4.137 \\
\hline 0.8 & 1.344 & 1.904 & 2.736 & 2.992 & 1.344 & 1.904 & 2.736 & 2.992 \\
\hline 0.9 & 0.594 & 0.936 & 1.242 & 1.782 & 0.594 & 0.936 & 1.242 & 1.782 \\
\hline
\end{tabular}

Table 4.5: Auctioneer's Revenue in TMCA and VCG for $K=2$ channels

\begin{tabular}{|l|l|l|l|l||l|l|l|l|}
\hline & \multicolumn{4}{|c||}{ TMCA } & \multicolumn{4}{c|}{ VCG } \\
\hline$b_{0} \backslash \mathrm{N}$ & 4 & 6 & 8 & 10 & 4 & 6 & 8 & 10 \\
\hline 0 & 0.247 & 0.467 & 0.595 & 1.311 & 0.247 & 0.458 & 0.584 & 1.246 \\
\hline 0.1 & 1.562 & 2.380 & 3.296 & 4.208 & 1.533 & 2.363 & 3.246 & 4.096 \\
\hline 0.2 & 2.579 & 3.877 & 5.212 & 6.437 & 2.579 & 3.877 & 5.193 & 6.413 \\
\hline 0.3 & 3.374 & 5.006 & 6.518 & 7.861 & 3.374 & 5.006 & 6.538 & 7.849 \\
\hline 0.4 & 3.807 & 5.729 & 7.387 & 9.236 & 3.809 & 5.722 & 7.395 & 9.214 \\
\hline 0.5 & 4.024 & 5.958 & 7.826 & 9.835 & 4.024 & 5.958 & 7.816 & 9.850 \\
\hline 0.6 & 4.047 & 5.618 & 7.731 & 9.703 & 4.047 & 5.617 & 7.731 & 9.703 \\
\hline 0.7 & 3.473 & 5.168 & 6.553 & 7.716 & 3.473 & 5.166 & 6.553 & 7.727 \\
\hline 0.8 & 2.480 & 3.648 & 5.072 & 6.785 & 2.480 & 3.648 & 5.072 & 6.800 \\
\hline 0.9 & 1.296 & 1.926 & 2.844 & 3.438 & 1.296 & 1.926 & 2.844 & 3.438 \\
\hline
\end{tabular}

Table 4.6: Auctioneer's Revenue in TMCA and VCG for $K=4$ channels 

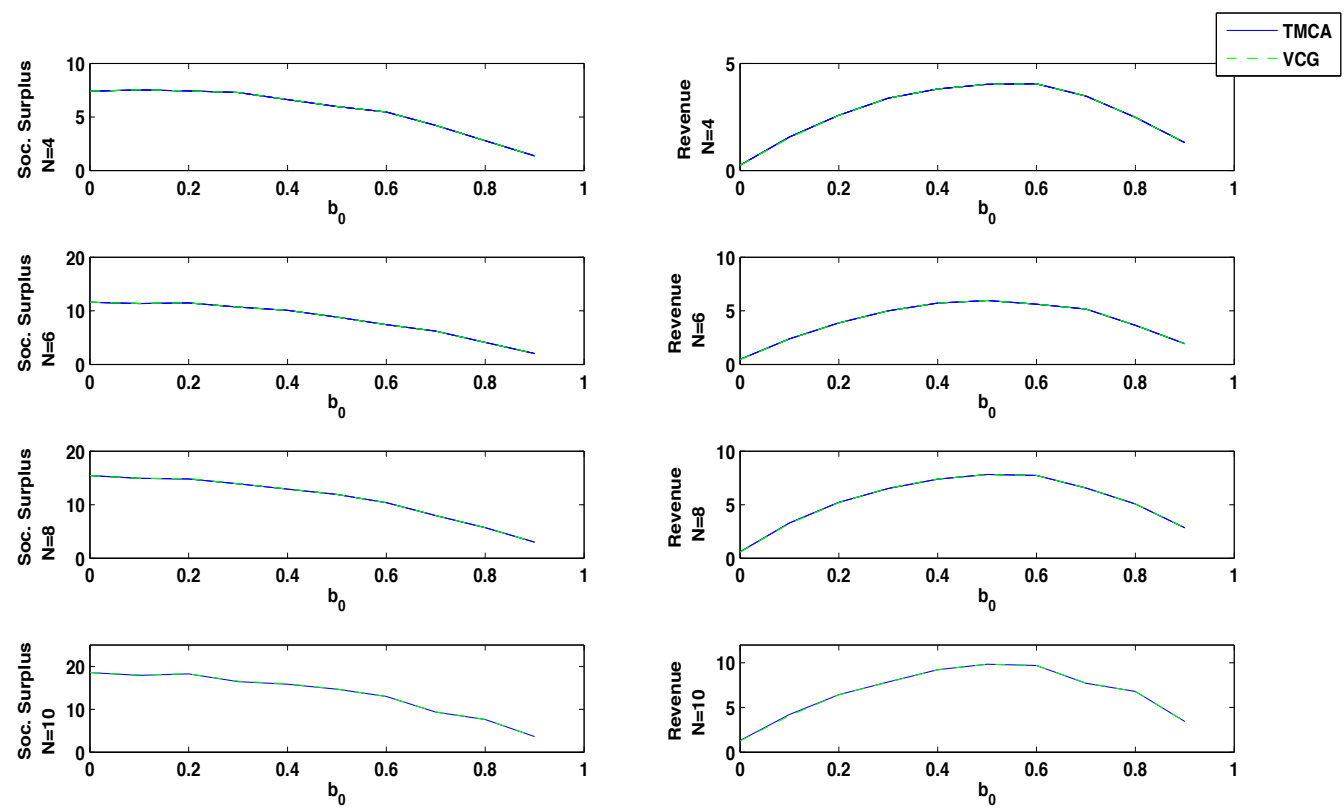

Figure 4.6: Social Benefit and Seller's Revenue (K=4)

\begin{tabular}{|l|l|l|l|l||l|l|l|l|}
\hline & \multicolumn{4}{|c||}{ TMCA } & \multicolumn{4}{c|}{ VCG } \\
\hline$b_{0} \backslash \mathrm{N}$ & 2 & 4 & 6 & 8 & 2 & 4 & 6 & 8 \\
\hline 0.1 & 0.002 & 0.005 & 0.007 & 0.009 & 0.049 & 0.161 & 0.421 & 1.050 \\
\hline 0.2 & 0.002 & 0.004 & 0.007 & 0.008 & 0.049 & 0.165 & 0.402 & 0.800 \\
\hline 0.3 & 0.002 & 0.004 & 0.006 & 0.009 & 0.053 & 0.165 & 0.377 & 0.908 \\
\hline 0.4 & 0.002 & 0.004 & 0.005 & 0.008 & 0.053 & 0.168 & 0.387 & 0.902 \\
\hline 0.5 & 0.002 & 0.003 & 0.005 & 0.007 & 0.049 & 0.165 & 0.372 & 0.733 \\
\hline 0.6 & 0.002 & 0.003 & 0.004 & 0.006 & 0.044 & 0.162 & 0.353 & 0.721 \\
\hline 0.7 & 0.001 & 0.002 & 0.004 & 0.005 & 0.044 & 0.151 & 0.349 & 0.615 \\
\hline 0.8 & 0.001 & 0.002 & 0.003 & 0.004 & 0.047 & 0.157 & 0.335 & 0.612 \\
\hline 0.9 & 0.001 & 0.001 & 0.002 & 0.002 & 0.045 & 0.148 & 0.323 & 0.519 \\
\hline
\end{tabular}

Table 4.7: CPU time of TMCA and VCG for $K=2$ channels

As expected computing time for VCG grows exponentially as the number of nodes increases. Tables 4.9 and 4.11 show social surplus and revenue of the auctioneer for TMCA and VCG when $K=2$ channels. Similarly, Tables 4.10 and 4.12 do the same for $K=4$ channels. These tables are summarized in Fig. 4.8.

Fig. 4.8 shows the social benefit and revenue of TMCA and Pivot for $K=4$, $N \in\{4,6,8,10\}$ and different values of $b_{0}$. Notice that the results are virtually the same for both mechanisms. Still, for small reserve prices, TMCA obtains a slightly higher revenue but a slightly lower social benefit. However this loss in social 


\begin{tabular}{|l|l|l|l|l||l|l|l|l|}
\hline & \multicolumn{4}{|c||}{ TMCA } & \multicolumn{4}{c|}{ VCG } \\
\hline$b_{0} \backslash \mathrm{N}$ & 2 & 4 & 6 & 8 & 2 & 4 & 6 & 8 \\
\hline 0 & 0.005 & 0.010 & 0.016 & 0.018 & 0.057 & 0.374 & 4.150 & 35.107 \\
\hline 0.1 & 0.004 & 0.010 & 0.014 & 0.018 & 0.093 & 0.389 & 3.381 & 36.820 \\
\hline 0.2 & 0.004 & 0.009 & 0.014 & 0.016 & 0.097 & 0.385 & 2.720 & 37.481 \\
\hline 0.3 & 0.004 & 0.008 & 0.013 & 0.017 & 0.099 & 0.405 & 3.387 & 24.595 \\
\hline 0.4 & 0.004 & 0.008 & 0.011 & 0.015 & 0.103 & 0.414 & 1.601 & 18.088 \\
\hline 0.5 & 0.004 & 0.006 & 0.010 & 0.013 & 0.104 & 0.437 & 1.647 & 16.031 \\
\hline 0.6 & 0.003 & 0.006 & 0.009 & 0.012 & 0.104 & 0.379 & 1.963 & 5.343 \\
\hline 0.7 & 0.002 & 0.005 & 0.007 & 0.009 & 0.099 & 0.332 & 1.152 & 2.308 \\
\hline 0.8 & 0.002 & 0.004 & 0.005 & 0.008 & 0.097 & 0.327 & 0.794 & 2.329 \\
\hline 0.9 & 0.001 & 0.002 & 0.004 & 0.005 & 0.096 & 0.340 & 0.741 & 1.157 \\
\hline
\end{tabular}

Table 4.8: CPU time of TMCA and VCG for $K=4$ channels

\begin{tabular}{|l|l|l|l|l||l|l|l|l|}
\hline & \multicolumn{4}{|c|}{ TMCA } & \multicolumn{4}{c|}{ VCG } \\
\hline$b_{0} \backslash \mathrm{N}$ & 4 & 6 & 8 & 10 & 4 & 6 & 8 & 10 \\
\hline 0 & 3.337 & 4.482 & 5.125 & 5.716 & 3.385 & 4.629 & 5.391 & 6.354 \\
\hline 0.1 & 3.173 & 4.386 & 5.159 & 5.962 & 3.207 & 4.493 & 5.410 & 6.506 \\
\hline 0.2 & 3.030 & 4.280 & 5.450 & 5.727 & 3.043 & 4.417 & 5.655 & 6.196 \\
\hline 0.3 & 3.071 & 4.487 & 5.126 & 5.745 & 3.114 & 4.594 & 5.430 & 6.217 \\
\hline 0.4 & 2.910 & 3.908 & 4.774 & 5.754 & 2.925 & 3.954 & 5.066 & 6.097 \\
\hline 0.5 & 2.660 & 4.143 & 4.866 & 5.491 & 2.673 & 4.268 & 4.944 & 5.777 \\
\hline 0.6 & 2.236 & 3.357 & 4.320 & 4.861 & 2.248 & 3.357 & 4.395 & 5.113 \\
\hline 0.7 & 2.124 & 2.973 & 3.764 & 4.356 & 2.124 & 3.024 & 3.779 & 4.498 \\
\hline 0.8 & 1.532 & 2.131 & 2.786 & 2.693 & 1.532 & 2.147 & 2.802 & 2.763 \\
\hline 0.9 & 0.662 & 1.194 & 1.691 & 1.913 & 0.662 & 1.212 & 1.691 & 1.931 \\
\hline
\end{tabular}

Table 4.9: Social Surplus of TMCA and VCG for $K=2$ channels (high density of users)

efficiency is small for practical scenarios as we have shown, in our experiments the maximum efficiency loss was of $10 \%$ (When $b_{0}=0$ and $N=10$ ). Reduction in CPU time however is of the order of $10^{3}$.

\subsection{Conclusion}

In this chapter, we studied auction mechanisms as promising tools to enable a dynamic and efficient re-allocation of spectrum channels in the short run. Although the VCG mechanism can be used for this channel reallocation, it is overly demanding from a computational standpoint. To solve this problem, we proposed TMCA, 

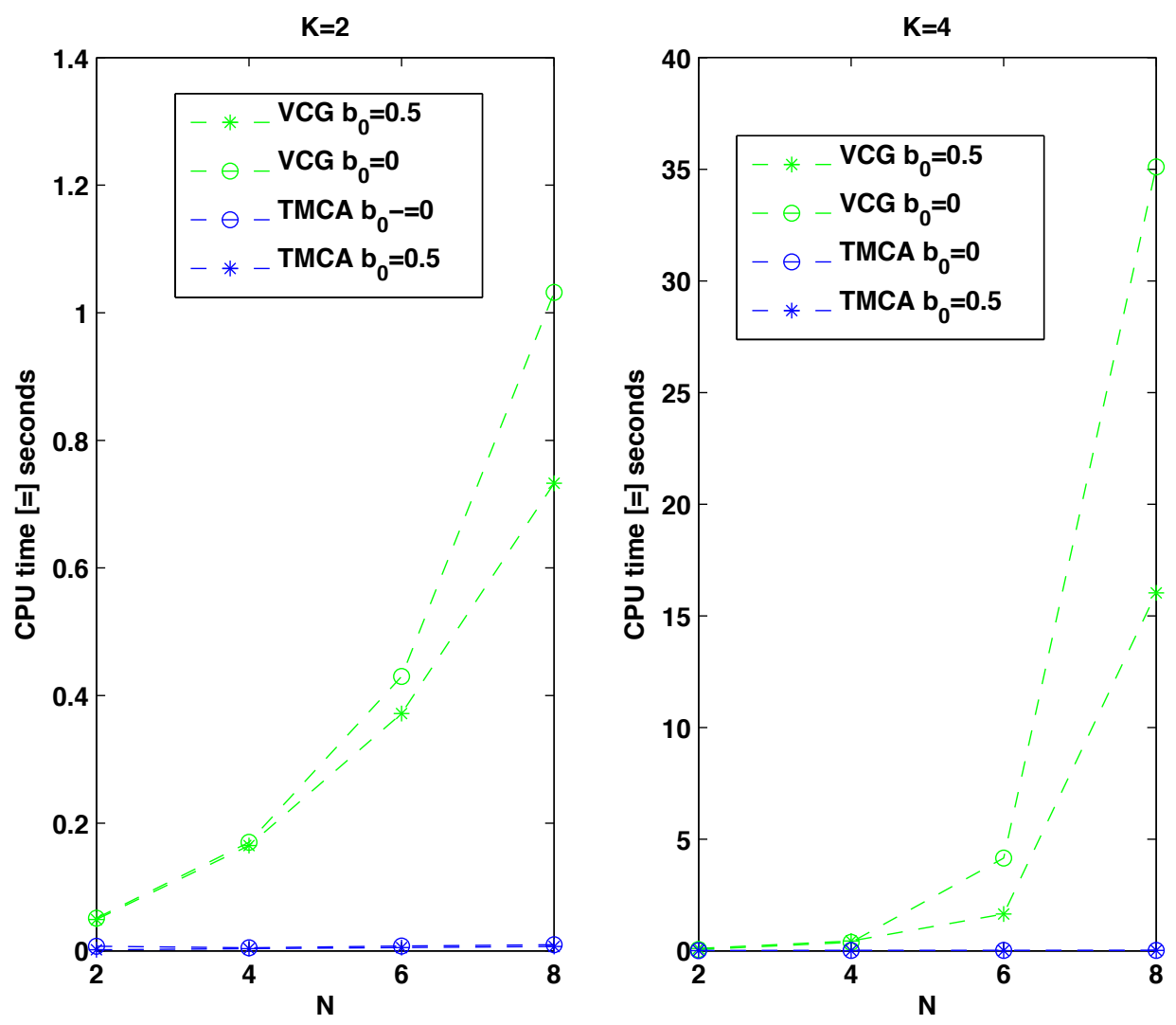

Figure 4.7: Exponential vs polynomial CPU time

\begin{tabular}{|l|l|l|l|l||l|l|l|l|}
\hline & \multicolumn{4}{|c|}{ TMCA } & \multicolumn{4}{c|}{ VCG } \\
\hline$b_{0} \backslash \mathrm{N}$ & 4 & 6 & 8 & 10 & 4 & 6 & 8 & 10 \\
\hline 0 & 6.427 & 8.719 & 10.806 & 12.094 & 6.551 & 8.997 & 11.466 & 13.212 \\
\hline 0.1 & 6.873 & 8.653 & 10.602 & 12.245 & 6.901 & 8.975 & 11.337 & 13.347 \\
\hline 0.2 & 6.643 & 8.494 & 10.570 & 11.950 & 6.719 & 8.844 & 11.206 & 13.098 \\
\hline 0.3 & 6.229 & 8.363 & 10.383 & 12.081 & 6.266 & 8.705 & 11.098 & 13.165 \\
\hline 0.4 & 6.034 & 8.643 & 10.275 & 11.480 & 6.105 & 8.781 & 10.781 & 12.389 \\
\hline 0.5 & 5.672 & 7.719 & 9.540 & 11.163 & 5.694 & 7.881 & 9.982 & 11.979 \\
\hline 0.6 & 5.001 & 7.258 & 8.488 & 10.502 & 5.015 & 7.341 & 8.843 & 11.082 \\
\hline 0.7 & 4.004 & 5.910 & 7.666 & 8.723 & 4.004 & 6.039 & 7.757 & 9.011 \\
\hline 0.8 & 3.160 & 3.883 & 5.821 & 7.136 & 3.160 & 3.916 & 5.920 & 7.286 \\
\hline 0.9 & 1.306 & 2.612 & 2.908 & 3.671 & 1.306 & 2.612 & 2.908 & 3.671 \\
\hline
\end{tabular}

Table 4.10: Social Surplus of TMCA and VCG for $K=4$ channels (high density of users)

a sealed-bid auction design that is incentive compatible in dominant strategies, individually rational and can be implemented in polynomial time. TMCA reduces the expected computational time at the expense of efficiency. For scenarios with 


\begin{tabular}{|l|l|l|l|l||l|l|l|l|}
\hline & \multicolumn{4}{|c||}{ TMCA } & \multicolumn{4}{c|}{ VCG } \\
\hline$b_{0} \backslash \mathrm{N}$ & 4 & 6 & 8 & 10 & 4 & 6 & 8 & 10 \\
\hline 0 & 0.643 & 1.116 & 2.000 & 2.658 & 0.577 & 0.934 & 1.584 & 2.206 \\
\hline 0.1 & 0.963 & 1.674 & 2.167 & 3.003 & 0.887 & 1.608 & 2.125 & 2.676 \\
\hline 0.2 & 1.253 & 1.974 & 2.760 & 3.230 & 1.248 & 1.950 & 2.584 & 3.012 \\
\hline 0.3 & 1.577 & 2.357 & 2.996 & 3.658 & 1.564 & 2.327 & 2.996 & 3.583 \\
\hline 0.4 & 1.786 & 2.376 & 3.056 & 3.780 & 1.763 & 2.356 & 3.114 & 3.817 \\
\hline 0.5 & 1.829 & 2.824 & 3.424 & 3.947 & 1.830 & 2.903 & 3.413 & 4.088 \\
\hline 0.6 & 1.724 & 2.554 & 3.315 & 3.813 & 1.736 & 2.552 & 3.345 & 3.948 \\
\hline 0.7 & 1.736 & 2.460 & 3.153 & 3.640 & 1.728 & 2.490 & 3.148 & 3.742 \\
\hline 0.8 & 1.361 & 1.889 & 2.510 & 2.390 & 1.361 & 1.902 & 2.523 & 2.452 \\
\hline 0.9 & 0.630 & 1.134 & 1.602 & 1.819 & 0.630 & 1.152 & 1.602 & 1.836 \\
\hline
\end{tabular}

Table 4.11: Auctioneer's Revenue in TMCA and VCG for $K=2$ channels (high density of users)

\begin{tabular}{|l|l|l|l|l||l|l|l|l|}
\hline & \multicolumn{4}{|c||}{ TMCA } & \multicolumn{4}{c|}{ VCG } \\
\hline$b_{0} \backslash \mathrm{N}$ & 4 & 6 & 8 & 10 & 4 & 6 & 8 & 10 \\
\hline 0 & 1.377 & 2.715 & 4.049 & 5.513 & 1.062 & 2.147 & 3.344 & 4.367 \\
\hline 0.1 & 1.889 & 3.315 & 4.491 & 5.922 & 1.787 & 3.036 & 4.060 & 5.122 \\
\hline 0.2 & 2.543 & 3.837 & 5.212 & 6.498 & 2.527 & 3.707 & 5.049 & 6.057 \\
\hline 0.3 & 3.084 & 4.630 & 5.899 & 7.087 & 3.106 & 4.537 & 5.770 & 7.029 \\
\hline 0.4 & 3.496 & 5.182 & 6.469 & 7.463 & 3.504 & 5.121 & 6.638 & 7.637 \\
\hline 0.5 & 3.825 & 5.286 & 6.697 & 8.001 & 3.823 & 5.354 & 6.877 & 8.305 \\
\hline 0.6 & 3.776 & 5.468 & 6.550 & 8.002 & 3.773 & 5.503 & 6.742 & 8.368 \\
\hline 0.7 & 3.292 & 4.814 & 6.330 & 7.263 & 3.292 & 4.917 & 6.370 & 7.484 \\
\hline 0.8 & 2.816 & 3.444 & 5.177 & 6.347 & 2.816 & 3.476 & 5.262 & 6.475 \\
\hline 0.9 & 1.242 & 2.466 & 2.754 & 3.492 & 1.242 & 2.466 & 2.754 & 3.492 \\
\hline
\end{tabular}

Table 4.12: Auctioneer's Revenue in TMCA and VCG for $K=4$ channels (high density of users)

low density of bidders this efficiency loss showed negligible while reductions in CPU time were of the order of $10^{2}$. For scenarios with high density of bidders, efficiency loss remained small (less than 10\%) while reduction in CPU time was of the order of $10^{3}$. We also characterized a revenue maximizing TMCA design in which a reserve price per unit is determined. In contrast to former auction designs, TMCA simultaneously achieves incentive compatibility in dominan strategies, individual rationality, and implementability in polynomial time when marginal channel valuations are not fixed. 

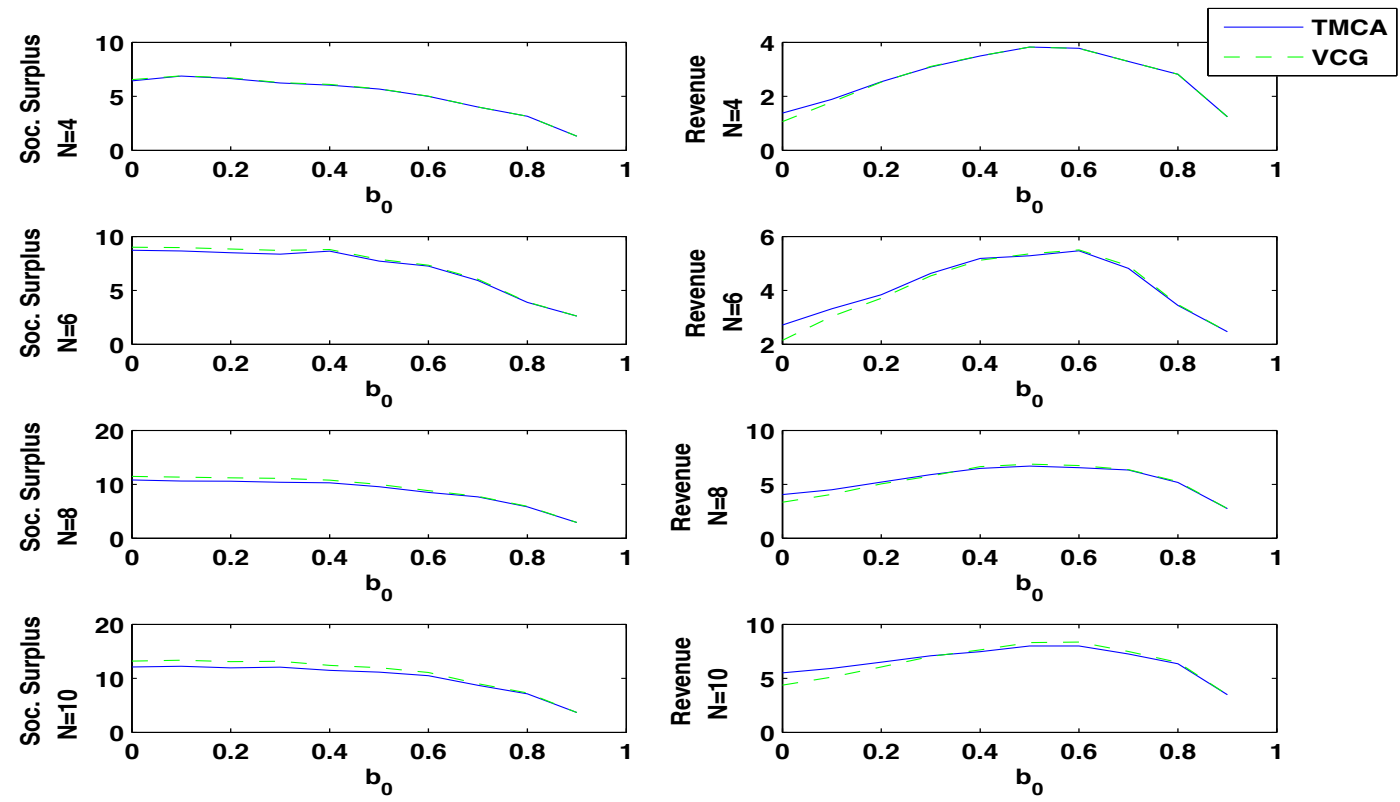

Figure 4.8: Social Benefit and Seller's Revenue (K=4) (high density of users)

\subsection{Appendix}

\subsubsection{Appendix: NP-Completeness of channel allocation}

In this subsection we prove that given valuations $\left\{\mathbf{v}_{n}: n \in \mathcal{N}\right\}$ finding the socially efficient allocation is an NP-complete problem. Consider the Chromatic Number Problem $(C N P)$ where given a natural $K>0$ and a graph $G(\mathcal{N}, \mathcal{E})$ where $\mathcal{N}$ is the set of nodes and $\mathcal{E}$ the set of edges, we want to know if it is possible to find a coloring of the graph using $\mathrm{K}$ or less colors such that no neighbor nodes receive the same color. Notice that we can solve the chromatic number problem by finding an optimal allocation $a^{*}\left(\hat{\mathbf{v}}_{1}, \ldots, \hat{\mathbf{v}}_{N}\right)$ if we define $\hat{\mathbf{v}}_{n, k}$ as follows:

$$
\hat{\mathbf{v}}_{n, k}=\left\{\begin{array}{cc}
1 & \text { if } k=1 \\
0 & \text { otherwise }
\end{array}\right.
$$

for all $n \in \mathcal{N}$. The solution to the chromatic number instance is affirmative iff each node receives one channel. Therefore the CNP is polynomially reducible to the optimal allocation problem. Since the former is NP-complete (see [18]), so is 
the latter.

\subsubsection{Appendix: Preallocation Phase and Feasibility}

In this section, we prove that the preallocation phase of TMCA delivers a matrix â such that for any $n \in \mathcal{N}$ and $k^{\prime} \leq K$ if $b_{n, k^{\prime}}>s_{n, k^{\prime}}$ then $\sum_{k=1}^{K} \hat{\mathbf{a}}_{n, k} \geq k^{\prime}$.

1. Suppose $b_{n, k^{\prime}}>s_{n, k^{\prime}}$ and $\sum_{k=1}^{K} \hat{\mathbf{a}}_{n, k}<k^{\prime}$ for some $n \in \mathcal{N}$ and $k^{\prime} \leq K$.

2. It follows that $n$ received at most $k^{\prime}-1$ channels, therefore his neighbors received at least $K-\left(k^{\prime}-1\right)$ channels. Thus $\mathcal{B}_{n}$ has at least $\left(K-k^{\prime}+1\right)$ elements greater than $b_{n, k^{\prime}}$.

3. Thus $s_{n, k^{\prime}}=\mathcal{B}_{n}\left(K-k^{\prime}+1\right)>b_{n, k^{\prime}}$, which contradicts the hypothesis in 1 . 


\subsection{Conclusions}

In this dissertation, we studied challenges faced by modern communication networks and the increasingly important role of mechanism design in order to overcome these challenges.

Modern communication networks are generally constituted by heterogeneous and profit maximizing users. If network users are required to follow predefined rules, these rules must be aligned with each user's individual objective.

The field of mechanism design offers the basic groundwork to align individual actions with more general objectives. The design of network algorithms with these considerations, however, is not an easy task. We have introduced "tailor-made" solutions to three different problems. In these problems, the network manager wanted to efficiently allocate network resources to a set of rational users with private information regarding their utility.

Chapter 2 studied the problem of efficient flow assignment in a wired network with capacity constraints. The network is prone to congestion externalities, which are function of the aggregate use of the links in the network. For this problem we propose Inertial Capacity Auction (ICA), an iterative auction design that implements the socially efficient flow assignment in $\epsilon$-dominant strategies.

Chapter 3 introduced the problem of a wireless up-link network where secondary users are serviced under restrictions imposed by the service quality of the primary users. The wireless nature of this problem poses heavily intertwined externalities that hinder the application the ICA. For this problem, we proposed Inertial Temperature Auction, an iterative auction design, that implements the socially efficient power profiles of secondary users $\epsilon$-dominant strategies.

Despite their structural differences, ICA and ITA are similar in nature. For example, both use externality prices and cumulative payments that allow each user to internalize the effect of his individual actions on his competitors' utility. Externality prices, however, are homogeneous in the ICA and heterogeneous in 
the ITA. This difference stems from the particularities of the wired and wireless systems.

A novel concept used in ICA and ITA is the inertia in the updates of users' profiles. This inertia helps users internalize the effects of the aggregate actions, which finally, guarantees convergence and prevents cycles in the joint profiles.

Chapter 4 addressed spectrum sharing when the spectrum has been partitioned into channels of semi-exclusive use. Here, channels can be reused under restrictions imposed by a conflict graph. For this problem we proposed Truthful Multichannel Auction, a one-shot auction design that is proved to be incentive compatible in dominant strategies and implementable in polynomial time.

There are several channel auctions that consider channel allocations, restricted by a conflict graph. However, to the best of our knowledge, TMCA is the first incentive compatible channel auction that can be implemented in polynomial time when the marginal channel valuations are not fixed.

The variety and sophistication of users sharing network resources represent considerable challenges to the administrators of modern networks. The field of mechanism design provides tools that are promising to solve many of these challenges. However, network algorithms based on the theory of mechanism design need to be "tailor made", bearing in mind not only the priorities of the design (e.g. efficiency, revenue of the network manager, etc.), but also the technical issues related to the resource sharing technology (eg. FDMA, CDMA, etc.). 


\section{Bibliography}

[1] Lawrence M. Ausubel, An efficient ascending-bid auction for multiple objects, The American Economic Review 94 (2004), 1452-1475.

[2] _ An efficient dynamic auction for heterogeneous commodities, American Economic Review 96 (2006), 602-629.

[3] Lawrence M. Ausubel and Paul Milgrom, The lovely but lonely vickrey auction, Stanford Institute for Economic Policy Research, 2004.

[4] J. Bae, E. Beigman, R. Berry, M. Honig, and R. Vohra, Sequential bandwidth and power auctions for distributed spectrum sharing, Selected Areas in Communications, IEEE Journal on 26 (2008), $1193-1203$.

[5] J. Bae, E. Beigman, R. Berry, M. L. Honig, and R. Vohra, A dynamic auction for spectrum sharing, Allerton conference on communication, control and computing, 2007.

[6] Sushil Bikhchandani and Joseph M. Ostroy, Ascending price vickrey auctions, Games and Economic Behavior 55 (2006May), no. 2, 215-241.

[7] H. Boche and S. Naik, Mechanism design and implementation theoretic perspective of interference coupled wireless systems, Communication, control, and computing, 2009. allerton 2009. 47th annual allerton conference on, 2009.

[8] Edward H. Clarke, Multipart pricing of public goods, Public Choice 11 (1971), no. 1, 1733.

[9] Federal Communications Commission, Principles for promoting the efficient use of spectrum by encouraging the development of secondary markets, FCC, 2000.

[10] Peter Cramton, Spectrum auction design, Review of Industrial Organization 42 (2013).

[11] A. Goldsmith, Wireless communications, Cambridge University Press, 2005.

[12] J.R. Green and J.J. Laffont, Incentives in public decision-making, Studies in public economics, North-Holland Pub. Co., 1979.

[13] Theodore Groves, Incentives in teams, Econometrica 41 (1973July), no. 4, 617-31.

[14] Jianwei Huang, Randall A. Berry, and Michael L. Honig, Auction-based spectrum sharing, Mob. Netw. Appl. 11 (2006), 405-418.

[15] ITU-T, Itu-infodev ict regulation toolkit, module 2: Competition and price, 2010.

[16] R. Jain, Network market design part i: bandwidth markets, Communications Magazine, IEEE 50 (November), no. 11, 78-83.

[17] Rahul Jain and Jean Walrand, An efficient nash-implementation mechanism for network resource allocation, Automatica 46 (2010), no. 8, 1276 -1283.

[18] R. Karp, Reducibility among combinatorial problems, Complexity of computer computations, 1972, pp. 85-103.

[19] Ian Kash, Rohan Murty, and David Parkes, Enabling spectrum sharing in secondary market auctions, Harvard University, 2010.

[20] F. P. Kelly, Charging and rate control for elastic traffic, European Transactions on Telecommunications. 8 (1997), no. 3, pp. 33-37. 
[21] F. P. Kelly, A. K. Maulloo, and D. K. H. Tan, Rate control for communication networks: Shadow prices, proportional fairness and stability, The Journal of the Operational Research Society 49 (1998), no. 3, pp. 237-252.

[22] V. Krishna, Auction theory, Elsevier Science, 2009.

[23] Steven H. Low and David E. Lapsley, Optimization flow control $i$ : basic algorithm and convergence, IEEE/ACM Trans. Netw. 7 (1999), no. 6, 861-874.

[24] Andreu Mas-Colell, Michael D. Whinston, and Jerry R. Green, Microeconomic theory, Oxford University Press, 1995.

[25] Paul Milgrom, Putting auction theory to work (Cambridge, ed.), Cambridge University Press, 2004.

[26] Roger Myerson, Optimal auction design, Mathematics of Operations Research 6 (1981), no. 1 , pp. $58-73$.

[27] David Parkes, Classic mechanism design. chapter 2, iterative combinatorial auctions: Achieving economic and computational efficiency ph.d. dissertation., Ph.D. Thesis, 2001.

[28] John G. Riley and William F. Samuelson, Optimal auctions, The American Economic Review 71 (1981), no. 3, pp. 381-392.

[29] Virgilio Rodriguez, Klaus Moessner, and Rahim Tafazolli, Market driven dynamic spectrum allocation over space and time among radio-access networks., Mobile Network Applications 11 (2006), 847-860.

[30] Michael H. Rothkopf, Thirteen reasons why the vickrey-clarke-groves process is not practical, Oper. Res. 55 (2007), 191-197.

[31] H. Shah Mohammadian and B. Abolhassani, Auction-based spectrum sharing for multiple primary and secondary users in cognitive radio networks, Sarnoff symposium, 2010 ieee, 2010.

[32] William Vickrey, Counterspeculation, auctions and competitive sealed tenders, The Journal of Finance 16 (1961), 8-37.

[33] Mark Walker, On the nonexistence of a dominant strategy mechanism for making optimal public decisions, Econometrica 48 (1980), no. 6, pp. 1521-1540.

[34] Hong Xu, Jin Jin, and Baochun Li, A secondary market for spectrum, Infocom, 2010 proceedings ieee, 2010, pp. $1-5$.

[35] Sichao Yang and B. Hajek, Vcg-kelly mechanisms for allocation of divisible goods: Adapting vcg mechanisms to one-dimensional signals, IEEE J.Sel. A. Commun. 25 (2007), no. $6,1237-1243$.

[36] Hui Yu, Lin Gao, Zheng Li, Xinbing Wang, and E. Hossain, Pricing for uplink power control in cognitive radio networks, Vehicular Technology, IEEE Transactions on $\mathbf{5 9}$ (2010), $1769-1778$.

[37] Xia Zhou, Sorabh Gandhi, Subhash Suri, and Haitao Zheng, ebay in the sky: strategyproof wireless spectrum auctions., Mobicom '08: Proceedings of., 2008, pp. 2-13.

[38] Xia Zhou and Heater Zheng, Trust: A general framework for truthful double spectrum auctions, Infocom 2009, ieee, 2009. 